\title{
Regulation of nitrogen fixation in Klebsiella pneumoniae: Nitrogen and oxygen signal perception by the negative regulator NifL
}

\author{
Dissertation \\ zur Erlangung des Doktorgrades \\ der Mathematisch-Naturwissenschaftlichen Fakultäten \\ der Georg-August-Universität zu Göttingen
}

vorgelegt von

Robert Thummer

aus Alfeld/Leine

Göttingen 2007 
D7

Referent: Prof. Dr. R. A. Schmitz-Streit

Korreferent: Prof. Dr. W. Liebl

Tag der mündlichen Prüfung: 31.10.2007 
Die vorliegende Arbeit wurde am Lehrstuhl für Allgemeine Mikrobiologie im Institut für Mikrobiologie und Genetik der Georg-August-Universität Göttingen und im Institut für Allgemeine Mikrobiologie der Christian-Albrechts-Universität zu Kiel angefertigt.

Finanziert wurde diese Arbeit aus Mitteln der Deutschen Forschungsgemeinschaft zum Thema „Untersuchungen zur Membranassoziation des negativen Regulators NifL in Klebsiella pneumoniae in Abhängigkeit von Sauerstoff- und Stickstoffanwesenheit“".

Teile dieser Arbeit wurden veröffentlicht in:

Thummer R. ${ }^{1}$, Stips J. ${ }^{1}$, Neumann M., Schmitz RA (2004). GlnK effects complex formation between NifA and NifL in Klebsiella pneumoniae. Eur. J. Biochem. 271: 3379-3388.

Thummer R., Klimmek O., Schmitz RA (2007). Biochemical studies of Klebsiella pneumoniae NifL reduction using reconstituted partial anaerobic respiratory chains of Wolinella succinogenes. J. Biol. Chem. 288: 12517-12526.

Außerdem wird voraussichtlich folgende Veröffentlichung in Kürze eingereicht:

Thummer R, Glöer J, Schmitz RA (2007). Analysis of membrane association of Klebsiella pneumoniae NifL: A mutational analysis of the regulatory protein.

${ }^{1}$ Thummer and Stips equally contributed to this work and are both considered first authors. 


\section{Acknowledgements}

Especially, I would like to thank Prof. Dr. Ruth Schmitz for patiently supervising and supporting the present thesis. Generally you deepened my interest in science and gave me an understanding of nitrogen regulation, in particular. In addition one or the other conversation has been an interesting enrichment for the future.

For spontaneously filling in as second examiner my thanks go to Prof. Dr. W. Liebl.

I would like to thank Prof. Dr. G. Gottschalk for generous support and helpful discussion.

I wish to thank PD Dr. Oliver Klimmek from the University of Frankfurt for giving me the opportunity to study electron transfer onto NifL.

Special thanks to all former and current members of the labs in Göttingen and Kiel: Anne, Christian, Claudia, Diana, Dominik, Filiz, Heike, Inke, Jasmin, Jens G., Jens T., Jessica, Julia B., Julia P., Jutta, Katrin, Liv-Lena, Nancy, Nicole, Rebekka, Roman and Tinki. It has always been great pleasure working with you in an inspiring and friendly atmosphere. When looking back I will also remember the great fun we had in spare time.

Further I like to thank all the people in the Institute for Microbiology and Genetics, Göttingen and the Institute for General Microbiology, Kiel. Especially I would like to thank members of the workshops who had always a quick solution for every technical problem.

Finally I would like to thank my family and all my friends who always encouraged and accompanied me. Above all I would like to thank my parents deeply for supporting me in every situation I had to cope with and for sparking my interest in science. 
TABLE OF CONTENTS

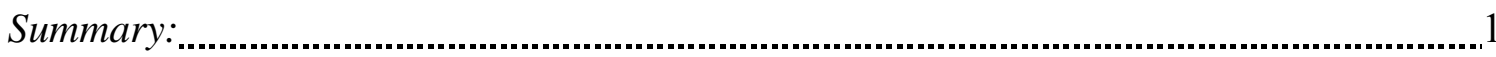

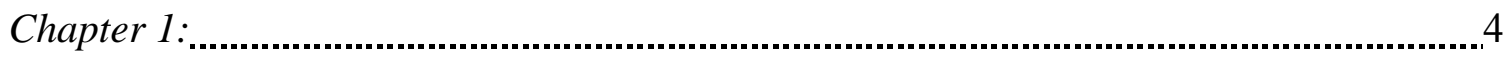

Introduction $\quad 4$

Chapter 2:

GInK effects complex formation between NifA and NifL in Klebsiella pneumoniae 11

$\begin{array}{ll}\text { Summary } & 11\end{array}$

Introduction $\quad 12$

Materials and Methods $\quad 13$

$\begin{array}{lr}\text { Results and Discussion } & 18\end{array}$

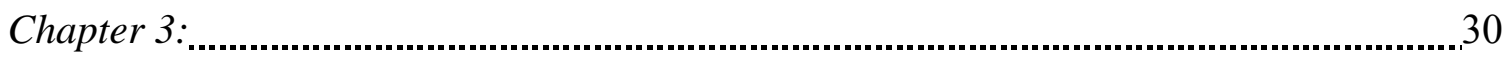

Biochemical studies of Klebsiella pneumoniae NifL reduction using reconstituted partial anaerobic respiratory chains of Wolinella succinogenes 30

$\begin{array}{ll}\text { Summary } & 30\end{array}$

Introduction $\quad 31$

Experimental procedures $\quad 33$

Results $\quad 36$

Discussion $\quad 47$

Footnotes $\quad 51$

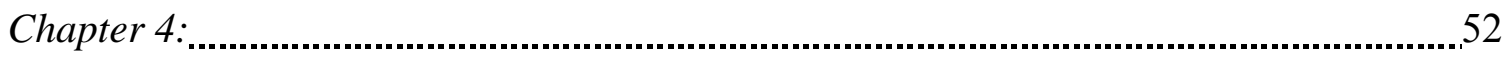

Analysis of membrane association of $K$. pneumoniae NifL: A mutational analysis of

$\begin{array}{ll}\text { the regulatory protein } & 52\end{array}$

$\begin{array}{lr}\text { Summary } & 52\end{array}$

Introduction $\quad 52$

Materials and Methods $\quad 54$

$\begin{array}{ll}\text { Results } & 59\end{array}$

$\begin{array}{ll}\text { Discussion } & 68\end{array}$

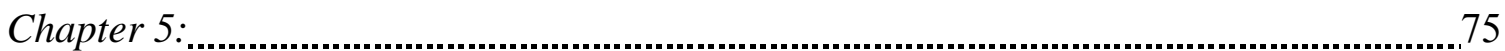

Conclusion $\quad 75$

Current working model for oxygen and nitrogen signalling in $K$. pneumoniae 81

$\begin{array}{ll}\text { Further studies } & 84\end{array}$

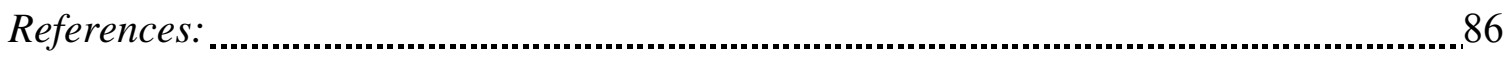

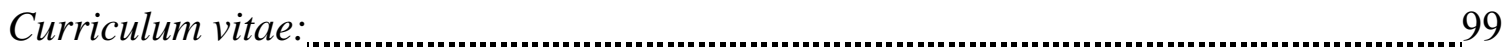




\section{Summary:}

- In Klebsiella pneumoniae transcription of nitrogen fixation (nif)-genes is regulated by the transcriptional activator NifA and its negative regulator NifL. Under conditions inappropriate for nitrogen fixation, NifL and NifA form inhibitory cytoplasmic complexes, thus preventing expression of nif-genes. Under conditions of nitrogen and oxygen absence, however, NifL is sequestered to the cytoplasmic membrane thus allowing cytoplasmic NifA to induce transcription of nif-genes.

- $\quad$ By means of pull-down experiments and quantitative Western Blot analysis it was demonstrated that NifA/NifL complexes formed in vivo in the presence of either molecular oxygen or combined nitrogen showed a stoichiometric 1:1 ratio. Interestingly this ratio was also found under nitrogen fixing conditions, however only $<1 \%$ of the amount of complexes formed under conditions inappropriate for nitrogen fixation was obtained, as the majority of NifL was sequestered to the cytoplasmic membrane.

- In order to confirm localization of NifL under different growth conditions, cells expressing MBP-NifL from an NtrC-independent promoter were grown under nitrogen and oxygen limitation and shifted to either nitrogen excess under anaerobic conditions $\left(+\mathrm{N} /-\mathrm{O}_{2}\right)$ or to aerobic nitrogen limiting growth $\left(-\mathrm{N} /+\mathrm{O}_{2}\right)$. Under derepressing conditions $\left(-\mathrm{N} /-\mathrm{O}_{2}\right)$ quantitative Western Blot analysis of membrane preparations showed that about $95 \%$ of MBP-NifL was membrane associated. However, after shifting the cultures to $+\mathrm{N} /-\mathrm{O}_{2}$ or $-\mathrm{N} /+\mathrm{O}_{2}$ conditions, $85 \%$ to $90 \%$ MBP-NifL was found in the cytoplasmic fraction. In order to verify that exclusively NifL localization is important for regulating nifgene transcription, in vitro transcription assays were conducted under anaerobic and aerobic conditions with anaerobically and aerobically synthesized MBP-NifL respectively. In both cases, strong inhibition of NifL on NifA was obtained independent of oxygen presence or absence. This clearly confirmed that the change of localization of NifL is the key regulatory mechanism of nitrogen fixation in $K$. pneumoniae and that complex formation between NifL and NifA is exclusively dependent on NifL located in the cytoplasm.

- For several nitrogen fixing organisms it has been shown that the PII-like protein GlnK is the primary sensor protein for the cellular nitrogen status and transduces the signal to the respective nif-regulatory systems. In case of bacteria only containing NifA, a direct effect on NifA activity has been demonstrated. However in K. pneumoniae GlnK might interact with NifL or NifA. Thus, GlnK was coexpressed from a plasmid with either MBP-NifL or MBP-NifA followed by pull-down experiments. Interestingly, GlnK was able to interact with both, MBP-NifL and MBP-NifA. The analysis of in vivo formed MBP-NifL/NifA 
complexes purified by pull-down experiments further showed that chromosomally expressed GlnK was present in the MBP-NifL/NifA complexes. This demonstrated for the first time that trimeric complexes between NifL/NifA and GlnK are formed in vivo. The ratio between the three proteins after coexpression from one plasmid was determined to be MBP-NifL : NifA : GlnK $\mathrm{H}_{3}=1.0: 0.86 \pm 0.1: 0.16 \pm 0.015$, indicating that the interaction between GlnK and NifL/NifA appears to be weak. These findings strongly indicate that the trimeric complex NifL/NifA/GlnK presumably reflects a transitional status of the nitrogen signal transduction to NifL/NifA.

- To further demonstrate that GlnK destabilizes the NifL/NifA complex, NifA-dependent transcription of nifH'-lacZ fusion was monitored in vivo. When cells were growing on $4 \mathrm{mM}$ ammonium as nitrogen source (resulting in nif-repression), the effect of additional NtrC independent expression of GlnK from a plasmid was studied. The observed significant nif-induction upon additional GlnK expression further confirms that additional GlnK leads to further dissociation of the inhibitory NifL/NifA complexes.

Furthermore localization analysis of MBP-NifL after coexpression with GlnK from a plasmid under nitrogen sufficiency and oxygen limitation showed that $95 \%$ of MBP-NifL was sequestered to the cytoplasmic membrane resulting in free cytoplasmic NifA. These findings clearly underline the destabilizing function of GlnK on the NifL/NifA inhibitory complexes. Unexpectedly, GlnK was also determined to be present in the membrane fractions indicating that GlnK might be sequestered to the cytoplasmic membrane in a NifL dependent manner.

- The reduction of the N-terminal bound FAD-cofactor of NifL by electrons derived from the reduced menaquinone pool under nitrogen fixing conditions results in membrane association of NifL. To study, whether electrons are directly transferred onto the FADcofactor or whether another membrane-bound oxidoreductase is the physiological electron donor for NifL reduction the potential electron transfer from a partially reconstituted respiratory chain of Wolinella succinogenes, a strictly anaerobic rumen bacterium, to NifL was analyzed spectroscopically. Using proteoliposomes reconstituted with Fdh and menaquinones of $W$. succinogenes oxidation of formate by Formate-dehydrogenase (Fdh) lead to the reduction of MBP-NifL. This finding strongly indicates that the electron transfer onto NifL occurs directly and unspecifically.

- $\quad$ The reduction rate of NifL bound FAD by the partial reconstituted anaerobic respiratory chain of $W$. succinogenes was shown to be directly dependent on the amount of both, Fdh and menaquinones. By doubling the amount of Fdh incorporated into proteoliposomes, the reduction of NifL was 4 -fold increased. The same effect was obtained by doubling the 
amount of menaquinones. Interestingly, by simultaneous doubling the amount of menaquinones and Fdh, NifL reduction rate increased 9 fold. The in vivo amount of reduced menaquinones is about 3 orders of magnitude higher compared to the amount of NifL molecules presumably allowing a much higher reduction rate than obtained in vitro.

- Sucrose-density gradient centrifugation analysis of MBP-NifL fully reduced by formate oxidation using the reconstituted partial anaerobic respiratory chain of $W$. succinogenes clearly demonstrated that reduced MBP-NifL associates with the proteoliposomes. In contrast, under aerobic conditions, oxidized MBP-NifL was not found to be present in the fractions containing proteoliposomes ( $32 \%$ sucrose) but present at much lower sucrose concentrations $(\sim 22 \%)$. As no further K. pneumoniae proteins are required for the sequestration of MBP-NifL to the proteoliposomes in vitro these findings suggest that in vivo membrane association of reduced NifL occurs unspecifically.

- To identify amino acids in the N-terminal domain of NifL, which are required for membrane association of NifL or in transducing the oxygen signal a screening system for randomly mutagenized nifL has been established. The nifL-gene under the control of the T7-promoter was mutated by PCR based mutagenesis, TA-cloned in pCRII and transformed into $K$. pneumoniae wild type. Under nitrogen fixing conditions, chromosomally expressed wild type NifL is sequestered to the cytoplasmic membrane. Mutations of plasmid encoded nifL resulting in amino acid changes which are essential for membrane association are no longer sequestered to the cytoplasmic membrane under nitrogen fixing conditions resulting in inhibition of NifA-activity (nif--phenotype).

- Overall, approximately 11.500 mutant clones from three independent mutagenesis assays were screened for a nif ${ }^{-}$-phenotype under nitrogen and oxygen limiting conditions. 67 mutants were identified, for which a nif ${ }^{-}$-phenotype was confirmed in several independent screening assays on solid and in liquid media.

- The plasmids of mutants with a confirmed nif - -phenotype were sequenced and the amino acid mutations analyzed. The crystal structure of the N-terminal PAS1 domain of A. vinelandii, which shows approximately $60 \%$ similarity to the PAS domain of K. pneumoniae NifL on amino acid level, was used to predict amino acid residues which are (i) presumably located on the protein surface or (ii) involved in coordinating the FADcofactor of NifL. Mutations Q57L and R80C were introduced separately into the plasmid encoded nifL-gene by site directed mutagenesis. The nif--phenotype of these single mutants could be confirmed in liquid medium. Further biochemical experiments concerning e.g. analyzing membrane association and FAD incorporation of these mutant proteins have to follow. 


\section{Chapter 1:}

\section{Introduction}

The highly energy demanding reduction of molecular nitrogen $\left(\mathrm{N}_{2}\right)$ to ammonium can also be accomplished biologically. This enzymatic process is called nitrogen fixation and is carried out by the enzyme complex nitrogenase. All known organisms containing this enzyme complex belong to the prokaryotes. Especially many species within the bacteria are able to fix molecular nitrogen (Dean and Jacobson, 1992; Fischer, 1994; Lobo and Zinder, 1992; Martinez-Argudo et al., 2005; Schmitz et al., 2002; Young, 1992) e.g. many symbiontic genera such as Rhizobia, Bradyrhizobia, Herbaspirillum and Azospirillum belonging to the $\alpha$ and $\beta$ proteobacteria. Besides there are also organisms known which are free living diazotrophs. The best studied are Azotobacter vinelandii and Klebsiella pneumoniae which both belong to the group of $\gamma$ proteobacteria and which both have a complex system of regulating transcription of genes involved in nitrogen fixation (nifgenes). Within the archaea the only known diazotrophs are within the methanogenic archea (Belay et al., 1984; Galagan et al., 2002; Kessler et al., 1997; Kessler et al., 1998; Leigh, 2000) such as Methanosarcina mazei (Deppenmeier et al., 2002) which are capable of nitrogen fixation.

Nitrogen fixation and regulation. Due to high energy demands required for biological nitrogen fixation - up to $40 \%$ of cellular produced ATP is dedicated for $\mathrm{N}_{2}$ reduction (Daesch and Mortenson, 1972; Upchurch and Mortenson, 1980) - and because the nitrogenase enzyme complex is irreversibly inactivated by molecular oxygen, synthesis and activity of nif-genes and the respective gene products are strictly regulated not to unnecessarily consume energy. Overall about 30 mole ATP are consumed per mole fixed molecular nitrogen corresponding to the fact that each electron transferred onto $\mathrm{N}_{2}$ and the following intermediate products is driven by consumption of 2 ATP-molecules (Burgess and Lowe, 1996; Halbleib and Ludden, 2000; Howard and Rees, 1996; Rees and Howard, 1999). The reaction catalyzed by the nitrogenase is summarized in the following equitation: $\mathrm{N}_{2}+8 \mathrm{H}^{+}+8 \mathrm{e}^{-} \rightarrow 2 \mathrm{NH}_{3}+\mathrm{H}_{2}$

The two environmental signals oxygen and nitrogen availability regulate transcription and activity of NifA which is the transcriptional activator for all nitrogen fixation (nif)-genes. Although the key enzyme for nitrogen fixation - nitrogenase - is present in all diazotrophs, 
the mechanisms of regulating nif-gene expression in response to oxygen and ammonium show a variety amongst the different organisms (Dixon, 1998; Fischer, 1994, 1996; Halbleib and Ludden, 2000; Schmitz et al., 2002). In many microorganisms NifA activity itself is oxygen sensitive ( $\alpha$ and $\beta$ proteobacteria) and can sometimes be directly influenced by combined nitrogen (Fischer, 1994, 1996; Steenhoudt and Vanderleyden, 2000). In contrast within the $\gamma$-proteobacteria a second regulatory protein plays an important role in $K$. pneumoniae and A. vinelandii. In these model organisms the negative regulator NifL, a flavoprotein, which is encoded in the nifLA operon (Dixon, 1998; Filser et al., 1983) antagonizes NifA and thus inhibits the transcription of nif-genes in the presence of oxygen and/or ammonium (Fig. 1.1). Interestingly the nifLA operon is differently regulated in K. pneumoniae and A. vinelandii. In contrast to Azotobacter, where nifL and nifA are constitutively expressed (Blanco et al., 1993), the nifLA operon of $K$. pneumoniae itself is regulated by the NtrB/NtrC-system which is a global two component regulatory system in enterobacteria responding to the intracellular nitrogen status (Drummond and Wootton, 1987).

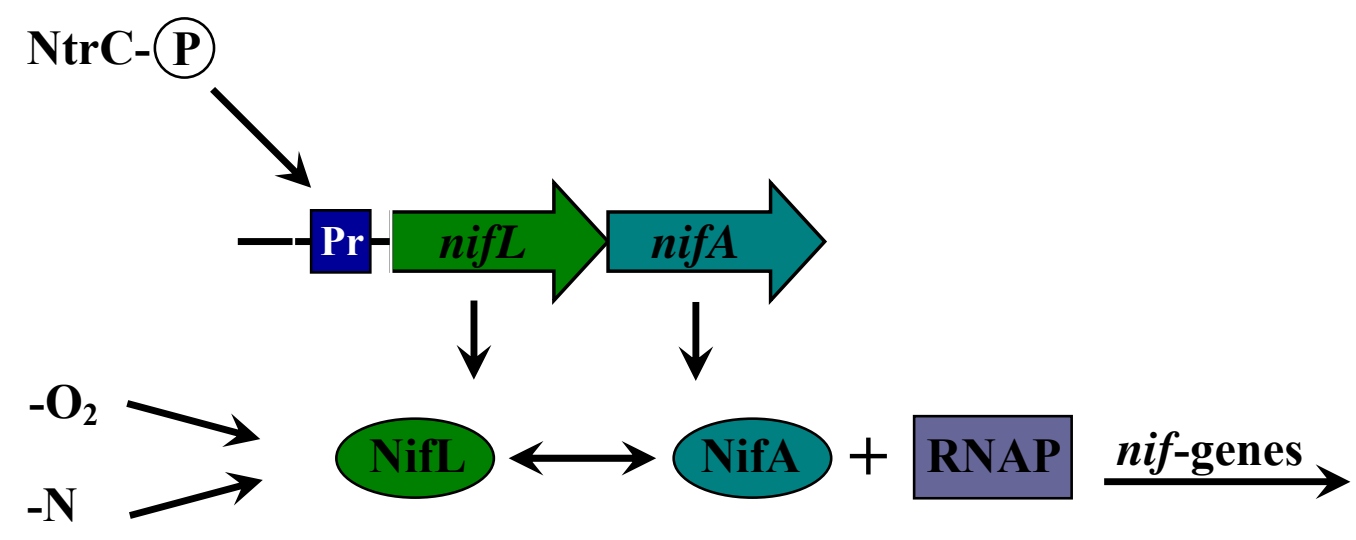

Figure 1.1: Organization of the nifLA-operon in $K$. pneumoniae. The phosphorylated global transcriptional activater $\mathrm{NtrC}$ activates the transcription of the nifLA-operon. Under conditions appropriate for nitrogen fixation the NifA/NifL complex dissociates and NifA activates the transcription of nif-genes in combination with the $\sigma^{54}$-RNA-Polymerase. 
Structure and function of the regulatory proteins NifL and NifA. The transcriptional activator for nif-gene expression consists of three domains with different functions. The amino $(\mathrm{N})$-terminal domain of NifA is required for regulation whereas the central domain and the carboxy (C)-terminal domain are involved in interaction with the RNA-Polymerase or in DNA-binding respectively (Drummond et al., 1990; Morett and Segovia, 1993). Under derepressing conditions $\left(-\mathrm{N} /-\mathrm{O}_{2}\right)$ NifA activates the nif-gene transcription in combination with the alternative $\sigma^{54}$ RNA-polymerase by contacting its binding sites 5 ' of the nif-promoter and the RNA-polymerase holoenzyme which is located at the $\sigma^{54}$ promoter region (Buck et al., 1987). This transcriptionally not active RNA-Polymerase in its closed complex needs to be activated by ATP or GTP hydrolysis which is catalyzed by NifA mediating the RNA-Polymerase into the open form and thus initiating the transcription of nif-genes (Austin et al., 1994; Hoover et al., 1990; Lee et al., 1993a; Morett and Buck, 1989). However, in the presence of either molecular oxygen or combined nitrogen the negative regulator NifL inhibits the initiation of transcription by direct inhibiton of NifA (Dixon, 1998; Hill et al., 1981; Merrick, 1982; Schmitz et al., 2002). NifL is composed of two domains, an N-terminal domain consisting of one (K. pneumoniae) or two (A. vinelandii) PAS domains, which binds the redox-sensitive FAD-cofactor. The C-terminal domain presumably interacts with the N-terminal domain of the target protein NifA as the C-terminal domain itself is sufficient to inhibit NifA activity in vitro and in vivo (Narberhaus et al., 1995). The two domains of NifL are connected by a hydrophilic interdomain so called Q-linker (Drummond and Wootton, 1987; Soderback et al., 1998). Interestingly, the C-terminal domain of NifL contains an adenosine nucleotide binding domain homologous to GHKL ATPases (Blanco et al., 1993) although it has been demonstrated for $K$. pneumoniae and $A$. vinelandii that NifL is neither involved in autophosphorylation nor in phosphotransfer to NifA (Austin et al., 1994; Lee et al., 1993b; Little et al., 2006; Schmitz et al., 1996). PAS domains as they occur in the N-terminal domain of NifL are known to be part of many regulatory proteins which are found in all kingdoms of life sensing e.g. oxygen, light or redox-changes (Taylor and Zhulin, 1999; Zhulin et al., 1997).

NifL inhibits NifA activity by direct protein-protein interactions as confirmed by immunological studies (Govantes et al., 1998; Henderson et al., 1989). Another line of evidence for direct protein-protein interaction between NifL and NifA has been demonstrated for $A$. vinelandii by showing in vitro complex formation by cochromatography and in vivo by using yeast two hybrid system experiments (Lei et al., 
1999; Money et al., 1999; Money et al., 2001). Furthermore in A. vinelandii different domains have been identified which play a crucial role in transmitting the signals molecular oxygen and combined nitrogen to NifA. Therefore the central domain of A. vinelandii NifL is involved in switching from the active form of NifL to the inactive form by conformational changes in response to the environmental signals perceived in the FAD containing PAS1 domain (oxygen) and the GHKL domain (nitrogen) (Little et al., 2006; Martinez-Argudo et al., 2004c).

Nitrogen signalling to the NifL/NifA system. One of the environmental signals which lead to transcriptionally active NifA is the absence of nitrogen. Upon nitrogen sufficiency NifA activity is repressed by NifL (Arnott et al., 1989; Blanco et al., 1993). As a consequence NifL has to sense the intracellular nitrogen status itself or the signal has to be transduced towards either NifL or the NifL/NifA complex. One candidate which is likely to sense the intracellular nitrogen status via the glutamine/glutamate and the 2-oxoglutarate pools and transducing the nitrogen signal to the NifL/NifA regulatory system is the PII-like protein GlnK (Atkinson and Ninfa, 1999; He et al., 1998; Merrick and Edwards, 1995; Schmitz, 2000; van Heeswijk et al., 1996; Xu et al., 1998). This small regulatory protein which shows high homology to GlnB is ubiquitously organized in one operon with the high affinity ammonium transporter $a m t B$ (Arcondeguy et al., 2001).

In $K$. pneumoniae the glnKamtB-operon is tightly controlled by the NtrB/NtrC two component system and is only expressed under nitrogen limitation. GlnK obviously plays an important role in the regulation of nitrogen fixation as genetic studies revealed that in a glnK mutant strains NifL can not release NifA activity under derepressing conditions thus inhibiting NifA activity and therefore the transcription of nif-genes although growing under nitrogen limiting conditions (Arcondeguy et al., 1999; He et al., 1998; Jack et al., 1999). Interestingly the covalent modification of GlnK under conditions of nitrogen limitation has not been shown to influence or be essential for nif-regulation in $K$. pneumoniae (Arcondeguy et al., 1999; He et al., 1998).

In $A$. vinelandii the glnKamtB-operon is expressed constitutively suggesting that the regulation through GlnK occurs differently to K. pneumoniae (Meletzus et al., 1998). As demonstrated by in vitro transcription experiments, $A$. vinelandii GlnK is not involved in derepressing NifA activity under nitrogen limiting conditions. Based on in vitro experiments Dixon and coworkers propose a model of nitrogen signal transduction which is different from other diazotrophs. They propose that the unmodified form of GlnK 
directly interacts with the C-terminal domain of NifL under nitrogen excess resulting in an activation of the inhibitory form of NifL (Little et al., 2000; Little et al., 2002; ReyesRamirez et al., 2000). However under nitrogen limiting conditions 2-oxoglutarate binds to the GAF domain of NifA leading to the dissociation of the inhibitory complexes (Little and Dixon, 2003). Furthermore GlnK is uridylylated upon these conditions und thus no longer able to interact with NifL (Little et al., 2006; Martinez-Argudo et al., 2004c, 2005). This specific interaction between GlnK and NifL in A. vinelandii was further underlined using yeast two-hybrid system experiments (Rudnick et al., 2002).

Interestingly, it was demonstrated for E. coli that the non uridylylated form of GlnK is highly membrane associated after shift experiments from nitrogen limitation to nitrogen sufficiency so the PII-like protein is no longer present for regulation in the cytoplasm (Coutts et al., 2002). Recently it was demonstrated for E. coli that this membrane association occurs in an AmtB dependent manner. As crystal structures of the AmtB-GlnK complex revealed only the non-uridylylated form of GlnK is able to adhere at AmtB with it's T-loop locking the pore for channelling ammonium into the cell (Conroy et al., 2007). In diazotrophs which are missing the negative regulator NifL there is strong evidence that PII proteins directly interact with the N-terminal domain of NifA in order to transduce the nitrogen signal onto the nif-regulation system (Arsene et al., 1999; Monteiro et al., 1999a; Monteiro et al., 1999b; Steenhoudt and Vanderleyden, 2000).

\section{Oxygen signalling to the NifL/NifA system.}

In K. pneumoniae as well as in A. vinelandii NifL transduces the oxygen signal towards NifA. Both proteins were biochemically characterized and it revealed that NifL is a flavoprotein containing a N-terminally bound FAD moiety (Hill et al., 1996; Klopprogge and Schmitz, 1999; Schmitz, 1997; Soderback et al., 1998). Nevertheless the mechanisms responsible for oxygen signal transduction significantly differ.

In K. pneumoniae the FAD-cofactor itself is not required for repression of NifA activity as demonstrated by in vitro transcription assays with NifL holoenzyme and NifL apoenzyme (Schmitz, 1997). Indeed it was demonstrated that the reduced FAD-cofactor of NifL which is located in the PAS domain of the protein acts indirectly as redox sensitive prosthetic group which is apparently required to transduce the signal of anaerobiosis (Hill et al., 1996; Little et al., 2000; Macheroux et al., 1998; Schmitz, 1997). The signal for the absence of oxygen is mediated by the global oxygen sensor fumarate nitrate reductase regulator (Fnr) via a signal transduction cascade onto NifL. Fnr consists of an oxygen 
sensitive [4Fe-4S] cluster and induces transcription of genes involved in anaerobic respiration e.g NADH-dehydrogenase and Formate-dehydrogenase resulting in the reduction of the membranous menaquinone pool of the cells under oxygen depletion (Green et al., 1996; Kiley and Beinert, 1998; Unden and Schirawski, 1997). The reduction of NifL occurs at the cytoplasmic membrane by electrons derived from this reduced menaquinone pool resulting in the sequestration of NifL to the cytoplasmic membrane (Grabbe et al., 2001; Grabbe and Schmitz, 2003; Klopprogge et al., 2002). This change in localization of the negative regulator NifL is apparently the key regulatory mechanism in K. pneumoniae nitrogen fixation that leads to free cytoplasmic NifA under nitrogen and oxygen limiting conditions thus initiating the transcription of nif-genes (Klopprogge et al., 2002).

In contrast to $K$. pneumoniae NifL reduction as well is essential for NifA activity in A. vinelandii, however the physiological electron donor is not known at present. Therefore Dixon and collegues propose that the transfer of electrons onto NifL bound FAD appears to be unspecifically achieved by reducing equivalents which occur during growth under anaerobic conditions (Dixon, 1998; Macheroux et al., 1998). As a matter of fact it is not likely in $A$. vinelandii that the reduced NifL is sequestered to the cytoplasmic membrane. For A. vinelandii another species specific mechanism for regulating nif-gene expression is discussed. Dixon and coworkers propose a working model for A. vinelandii NifL in which a conformational change of NifL induced in response to the reduction of the FAD moiety mediated probably by the PAS1 domain under anaerobic conditions appropriate for nitrogen fixation (Little et al., 2006; Little et al., 2007; Martinez-Argudo et al., 2004c). Additionally the intracellular 2-oxoglutarate level which presumably interacts directly with the N-terminal GAF domain of NifA stimulates the release of NifL. Non-uridylylated GlnK and adenosine nucleotides however impair the conformational change of NifL thus enforcing inhibition of nif-gene expression (Little et al., 2002; Little and Dixon, 2003; Martinez-Argudo et al., 2004a, b). 


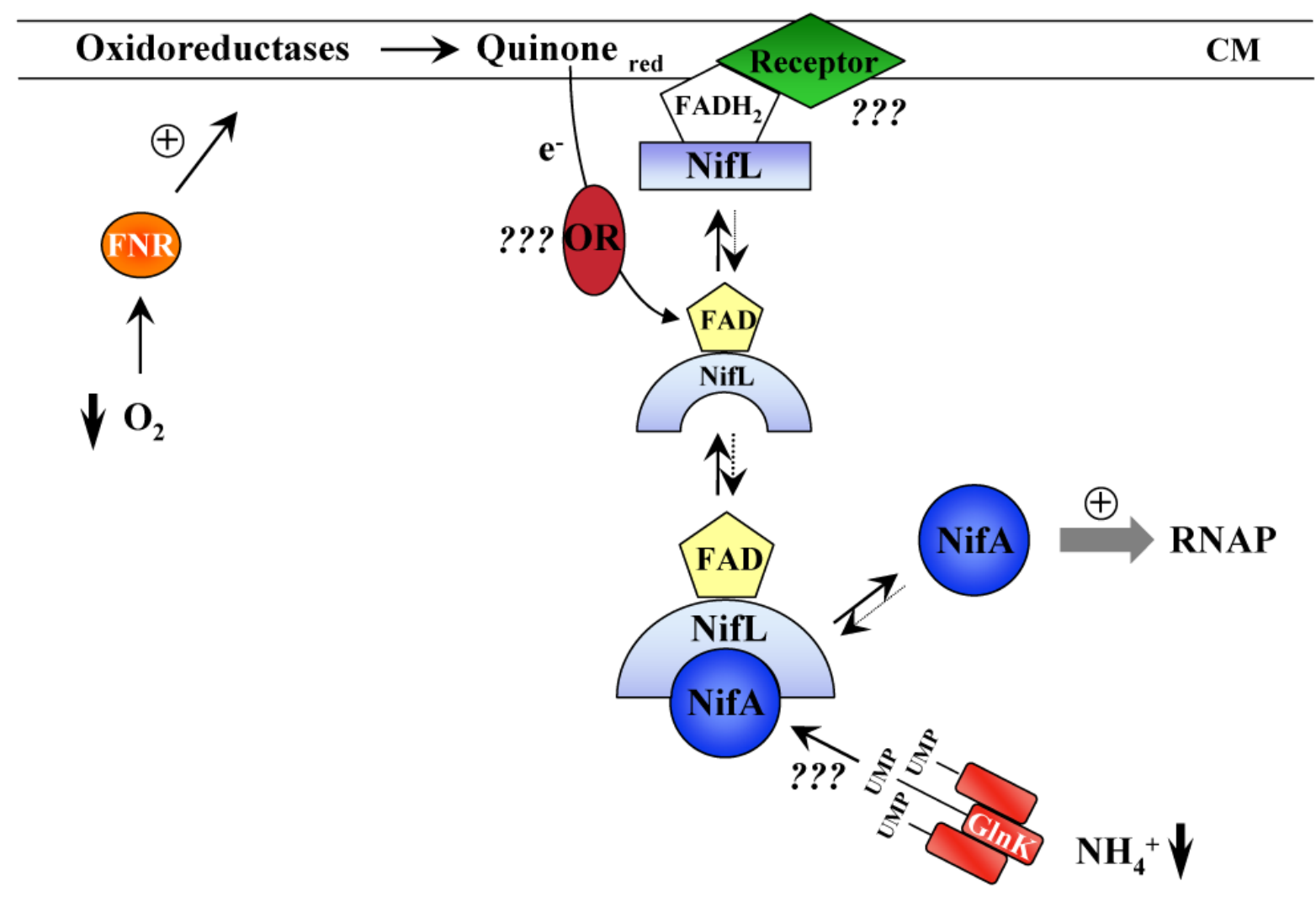

Figure. 1.2: Model for oxygen and nitrogen signal transduction to the NifA/NifL regulatory system in $K$. pneumoniae

The goal of this work was to get deeper insights into nitrogen and oxygen signal transduction to the NifL/NifA regulatory system of K. pneumoniae (Fig. 1.2). In former studies it has been shown that the electrons for NifL reduction are derived from the reduced menaquinone pool but it is not known whether the electron transfer onto the FADcofactor of NifL occurs unspecifically or whether a K. pneumoniae specific oxidoreductase is involved. Besides, it has not been excluded yet that a membrane associated receptor protein is required for membrane association of NifL. Furthermore the mechanism of nitrogen signal transduction onto the NifL/NifA regulatory system has not been sufficiently clarified. Therefore the focus of this thesis was to (i) biochemically analyze complex formation of the regulatory proteins NifL, NifA and GlnK under various growth conditions (Chapter 2) and (ii) to further characterize the FAD reduction of NifL in vitro (Chapter 3). (iii) Furthermore a genetic approach to identify crucial amino acid residues for oxygen signal transduction of NifL was performed (Chapter 4). 
Chapter 2:

\section{GInK effects complex formation between NifA and NifL in Klebsiella pneumoniae}

\section{Summary}

In Klebsiella pneumoniae, the nif specific transcriptional activator NifA is inhibited by NifL in response to molecular oxygen and ammonium. Here, we demonstrate complex formation between NifL and NifA (approximately 1:1 ratio), when synthesized in the presence of oxygen and/or ammonium. Under oxygen- and nitrogen-limitation, significant but fewer NifL/NifA-complexes (approximately $1 \%$ ) were formed in the cytoplasm as a majority of NifL was sequestered to the cytoplasmic membrane. These findings indicate that inhibition of NifA in the presence of oxygen and/or ammonium occurs via direct NifL interaction and formation of those inhibitory NifL/NifA-complexes appears to be directly and exclusively dependent on the localization of NifL in the cytoplasm. We further observed evidence that the nitrogen sensory protein GlnK forms a trimeric complex with NifL and NifA under nitrogen limitation. Binding of GlnK to NifL/NifA was specific, however, the amount of GlnK within these complexes was small. Finally, two lines of evidence were obtained that under anaerobic conditions but in the presence of ammonium additional NtrC-independent GlnK synthesis inhibited the formation of stable inhibitory NifL/NifA-complexes. Thus, we propose that the NifL/NifA/GlnK-complex reflects a transitional structure and hypothesize that under nitrogen-limitation, GlnK interacts with the inhibitory NifL/NifA-complex, resulting in its dissociation. 


\section{Introduction}

Nitrogen-fixing microorganisms tightly control both synthesis and activity of nitrogenase in response to oxygen and nitrogen availability, because of the high energy demands of nitrogen fixation and the oxygen sensitivity of nitrogenase (Burgess and Lowe, 1996; Rees and Howard, 1999). Transcription of the nitrogen fixation (nif) genes in diazotrophic bacteria is, in general, mediated by the activator protein NifA in combination with the alternative $\sigma^{54}$-RNA polymerase (Hoover et al., 1990; Morett and Buck, 1989). In the freeliving Klebsiella pneumoniae, Azotobacter vinelandii, and Azoarcus sp. BH72, NifA transcriptional activity is regulated by a second regulatory protein, NifL, which inhibits NifA in response to external molecular oxygen and ammonium (Egener et al., 2002; Hill et al., 1981; Hill et al., 1996; Merrick, 1982). This inhibition of NifA activity by NifL apparently occurs via direct protein-protein interaction, which is implied by evidence from immunological studies in K. pneumoniae (Henderson et al., 1989), and is consistent with recent studies for $A$. vinelandii using the yeast-two-hybrid system and in vitro analysis of complex formation between NifL and NifA (Lei et al., 1999; Little and Dixon, 2003; Money et al., 1999; Money et al., 2001; Reyes-Ramirez et al., 2002).

Under nitrogen limitation, NifL allows NifA activity only in the absence of oxygen, when its FAD-cofactor is reduced (Hill et al., 1996; Macheroux et al., 1998; Schmitz, 1997). Recently, we have shown that in K. pneumoniae, NifL is membrane-associated under simultaneous anaerobic and nitrogen-limited conditions, but is in the cytosolic fraction when in the presence of oxygen or sufficient nitrogen (Klopprogge et al., 2002). We further demonstrated that membrane association of NifL depends on NifL reduction at the cytoplasmic membrane by electrons derived from the reduced quinone pool (Grabbe et al., 2001; Grabbe and Schmitz, 2003). These findings indicate that sequestration of NifL to the cytoplasmic membrane under derepressing conditions appears to be the main mechanism for regulation of cytoplasmic NifA activity by NifL.

Recent genetic evidence strongly suggests that the nitrogen status of the cells is transduced towards the NifL/NifA regulatory system by the GlnK protein, a paralogue PII-protein (Arcondeguy et al., 1999; Arcondeguy et al., 2000; He et al., 1998; Jack et al., 1999; Little et al., 2000). Interactions between A. vinelandii GlnK and NifL were recently demonstrated using the yeast-two-hybrid system, and in vitro studies indicated that the non-uridylylated form of $A$. vinelandii GlnK activates the inhibitory function of NifL under nitrogen excess by direct protein-protein interaction (Little et al., 2002; Rudnick et al., 
2002). Under nitrogen limitation, however, the inhibitory activity of $A$. vinelandii NifL appears to be relieved by elevated levels of 2-oxoglutarate (Little et al., 2000; Little and Dixon, 2003; Reyes-Ramirez et al., 2001). In contrast to A. vinelandii, in K. pneumoniae the relief of NifL inhibition under nitrogen limitation depends on GlnK, the uridylylation state of which appears not to be essential for its nitrogen signaling function (Arcondeguy et al., 1999; Arcondeguy et al., 2000; He et al., 1998; Jack et al., 1999). We have recently shown that in the absence of GlnK, K. pneumoniae NifL was located in the cytoplasm and inhibited NifA activity under derepressing conditions (Klopprogge et al., 2002). However, it is currently not known how GlnK influences the localization of NifL in response to the nitrogen status and whether GlnK interacts directly with NifL or NifA, or affects the NifL/NifA-complex formation. In order to address those questions, we analyzed in vivo complex formation between the regulatory proteins after coexpression under various nitrogen and oxygen availabilities. During these studies we obtained evidence for the presence of an intermediate NifL/NifA/GlnK-complex, which is to our knowledge the first report for an in vivo formation of a NifL/NifA/GlnK-complex.

\section{Materials and Methods}

Bacterial Strains. The bacterial strains used in this work were Klebsiella pneumoniae M5al (wild type) and K. pneumoniae UN4495 ( $\phi(n i f K-l a c Z) 5935$ Alac-4001 his D4226 $G a l^{r}$ ) (MacNeil et al., 1981). Plasmid DNA was transformed into Klebsiella pneumoniae cells by electroporation.

Construction of plasmids: Plasmid pRS201 contains the K. pneumoniae nifLA operon, 5' fused to the Escherichia coli malE gene in pMAL-c2 (New England Biolabs, UK) which is under the control of the tac promoter. The plasmid was constructed as follows: A $3.1 \mathrm{kbp}$ PCR fragment carrying nifLA was generated using chromosomal K. pneumoniae DNA as template and a set of primers, which were homologous to the nifLA flanking $5^{\prime}$ and 3 '-regions with additional EcoRI and HindIII synthetic restriction recognition sites (underlined) (5'CACACAGGAAACAGAATTCCCGGG3', sense primer (NifLEcoRI); 5'CAATGTCCTGAAGCTTACATAAGGCTTCAC3', antisense primer (NifAHindIII). The $3.1 \mathrm{kbp}$ PCR product was cloned into the EcoRI and HindIII sites of pMAL-c2, resulting in malE fused to nifLA with one additional amino acid (Ala) preceding the 
methionine of NifL. The correct insertion was analyzed by sequencing. Plasmids encoding MBP-NifL (pRS180), MBP-NifA (pRS158), and MBP-NifL plus NifA (pRS209), in addition to K. pneumoniae GlnK under the control of the tac promoter, were constructed as follows: Plasmids pRS163, pRS98 and pRS205 were constructed by inserting a tetracycline-resistance cassette (de Lorenzo et al., 1990) into the HindIII site of plasmids pJES794, pJES597, and pRS201 encoding MBP-NifL, MBP-NifA, and MBP-NifL plus NifA, respectively (Lee et al., 1993a; Narberhaus et al., 1995) ; this work). A 0.4 kbp PCR fragment carrying $g \ln K$ under the control of the tac promoter was generated using pRS155 (Ehlers et al., 2002) as template and a set of phosphorylated primers: sense primer (pKK223-3F, 5'GACCACCGCGCTACTGCC3') and antisense primer (pKK223-3R, 5'GATGCCGGCCACGATGCG3'). This $0.4 \mathrm{kbp}$ PCR fragment was cloned into the ScaI site located inside the ampicillin resistance gene (bla) in pRS163, pRS98, and pRS205 resulting in pRS180 (MBP-NifL plus GlnK), pRS158 (MBP-NifA plus GlnK), and pRS209 (MBP-NifL plus NifA plus GlnK), respectively. pRS192 was constructed by inserting the $0.4 \mathrm{kbp}$ PCR fragment carrying $g \ln K$ under the control of the tac promoter generated as mentioned above into the SacI and PstI site of pMAL-c2 (New England Biolabs, UK) and the tetracycline-resistance cassette into the HindIII site. pRS239 was obtained by inserting the tetracycline-resistance cassette into the HindIII site of pRS155, coding for $g \operatorname{lnK}$ under the control of the tac promoter.

Growth conditions. K. pneumoniae strains were grown aerobically or anaerobically at $30{ }^{\circ} \mathrm{C}$ in minimal medium supplemented with either $4 \mathrm{mM}$ glutamine (nitrogen limitation) or $10 \mathrm{mM}$ ammonium (nitrogen sufficiency) as the sole nitrogen source and $1 \%$ sucrose as the sole carbon source (Schmitz et al., 1996). For anaerobic growth conditions in closed bottles with molecular nitrogen $\left(\mathrm{N}_{2}\right)$ as gas phase, the medium was supplemented with $0.3 \mathrm{mM}$ sulfide and $0.002 \%$ resazurin to monitor anaerobiosis. Precultures of the 1-1 anaerobic main cultures were grown overnight in closed bottles with $\mathrm{N}_{2}$ as gas phase in the same medium but lacking sulfide and resazurin. Aerobic 1-1 cultures were incubated in 2-1 flasks with vigorous shaking (130 rpm).

Cell extracts and purification of proteins. MBP-NifL and MBP-NifA was synthesized at $30^{\circ} \mathrm{C}$ under nitrogen limitation or sufficiency in K. pneumoniae carrying pJES794 (Narberhaus et al., 1995) and pJES597 (Lee et al., 1993a), respectively. Expression of fusion protein was induced from the tac promoter for $2 \mathrm{~h}$ with $100 \mu \mathrm{M}$ isopropyl- $\beta-\mathrm{D}$ - 
thiogalactopyranoside (IPTG) when cultures reached an O.D.600 $=0.6$. After disruption of cells in breakage (B) buffer and centrifugation at 20,000 $\mathrm{x}$ g, fusion proteins were purified from the supernatant by amylose affinity chromatography (Schmitz, 1997). Expression and purification of K. pneumoniae GlnK and E. coli GlnD $\Delta C$ were carried out as described recently (Ehlers et al., 2002). Purified GlnK was modified in vitro by uridylylation with E. coli $\mathrm{GlnD} \Delta C$ and the modification was investigated in non-denaturating polyacrylamide gels as recently described (Ehlers et al., 2002; Forchhammer and Hedler, 1997).

Complex formation assays with purified proteins. To analyze whether purified GlnK interacts with NifL or NifA, a binding assay using affinity chromatography was employed. Reactions were carried out in B-buffer in a total volume of $230 \mu 1$ in the presence or absence of MgATP $(1 \mu \mathrm{M}), \operatorname{MgADP}(1 \mu \mathrm{M})$ or $\alpha$-ketoglutarate $(10 \mu \mathrm{M})$. Purified MBPNifL, MBP-NifA, unmodified GlnK and uridylylated GlnK were generally used at $3 \mu \mathrm{M}$ in the reactions; the concentration of the GlnK fractions were calculated in terms of the trimer. After preincubation for $10 \mathrm{~min}$ at $30{ }^{\circ} \mathrm{C}, 500 \mu \mathrm{l}$ of amylose resin (New England Bioloabs, UK) equilibrated with B-buffer was added to the mixtures followed by an additional incubation for $20 \mathrm{~min}$ at room temperature. Non-binding protein was subsequently washed from the columns with B-buffer and the bound material was then eluted from the column with B-buffer containing $10 \mathrm{mM}$ maltose. Aliquots of the wash and elution fractions were separated on a denaturing $12.5 \%$ polyacrylamide gel, which was subsequently silver stained. The elution fractions were further analyzed by Western blot analysis using polyclonal antibodies raised against $K$. pneumoniae MBP-NifA, MBP-NifL or GlnK to detect small amounts of proteins.

\section{Isolation and characterization of complexes formed in vivo by affinity chromatography.}

Coexpression of malE-nifLA, malE-nifL plus glnK, malE-nifA plus glnK, and malE-nifLA plus $g \ln K$ were induced with $100 \mu \mathrm{M}$ IPTG at an optical density O.D.600 between 0.5 and 0.6 in K. pneumoniae strain M5a1 carrying pRS201, pRS180, pRS158 and pRS209 respectively. 1-1 main cultures were grown under aerobic or anaerobic conditions in the presence of $10 \mathrm{mM}$ ammonium or $4 \mathrm{mM}$ glutamine, see growth conditions. The respective growth and synthesis conditions were maintained until cell breakage, if not stated otherwise (e.g. in shift experiments). In general, purification of complexes subsequently followed directly after cell harvest without any storage at lower temperatures. Preparation of cell extracts in B-buffer and all following purification steps were performed in the 
presence of the protease inhibitor cocktail for bacterial cell extracts (Sigma, Aldrich). Depending on the synthesis conditions, cell extract preparation and purification of the fusion proteins from the $20,000 \mathrm{x} g$ supernatant by amylose affinity chromatography was performed either under aerobic conditions or under anaerobic conditions with a nitrogen atmosphere and inside an anaerobic chamber using anaerobic buffers supplemented with $2.0 \mathrm{mM}$ dithiothreitol (Schmitz, 1997). The respective wash and elution fractions were analyzed by gel electrophoresis and silver staining.

\section{Quantification of NifL, NifA and GInK in isolated complexes by Western blot analysis.}

After purification of potential complexes, proteins from the respective elution fractions were separated on denaturating polyacrylamide gels and transferred to nitrocellulose membranes (BioTrace ${ }^{\circledR}$ NT, Pall Life Science) (Sambrook, 1989). Membranes were exposed to specific polyclonal rabbit antisera directed against the MBP-NifL, MBP-NifA, GlnB or GlnK protein of K. pneumoniae. The primary antibodies were used in a high dilution range, conditions under which cross-reaction with other proteins are negligible. Protein bands were detected with secondary antibodies directed against rabbit immunoglobulin $\mathrm{G}$ and coupled to horseradish peroxidase (BioRad Laboratories) and visualized using the ECLplus system (Amersham Pharmacia) with a fluoroimager (Storm, Molecular Dynamics). The protein bands of the complexes were quantified for each growth condition from at least three independent cultures using the ImageQuant v1.2 software (Molecular Dynamics) and known amounts of the respective purified control proteins, which were simultaneously detected and quantified with the respective complex fraction on the same membrane for each experiment. Quantification of purified proteins MBP-NifL and MBP-NifA was linear within absolute amounts of 0.06 to $0.25 \mu \mathrm{g}$ per lane and GlnK within 0.01 to $0.14 \mu \mathrm{g}$. All quantifications of proteins have been performed within this linear range of the detection system. The relative amounts of GlnK in complexes are in general stated in terms of the trimeric GlnK protein $\left(\mathrm{GlnK}_{3}\right)$. Degradation of MBP-NifL and MBP-NifA in the elution fraction was frequently observed, as was the case for purified standard proteins. This degradation is based upon protein instability even under low temperature. As other proteins within the isolated complexes were not detected by SDSPAGE analysis and silver staining, the fusion protein and the major degradation products detected by the immunoblot were quantified together, if degradation occurred. 
B-Galactosidase assay. NifA-mediated activation of transcription from the nifHDK promoter in K. pneumoniae UN4495 and UN4495 carrying pRS239 was monitored by measuring the rate of $\beta$-galactosidase synthesis during exponential growth (units per $\mathrm{ml}$ per cell turbidity at $600 \mathrm{~nm}\left(\mathrm{OD}_{600}\right)$ (Schmitz et al., 1996)). Inhibitory effects of NifL on NifA activity in response to ammonium were assessed by virtue of a decrease in nifH expression.

In vitro transcription assay. Single cycle transcription assays were performed at $30{ }^{\circ} \mathrm{C}$ with purified $\sigma^{54}$ RNAP as described by Narberhaus et al. (Narberhaus et al., 1995) using $1.0 \mu \mathrm{M}$ central domain of NifA (cdNifA), $\sigma^{54}$ RNAP (60 nM core polymerase and $100 \mathrm{nM}$ $\sigma^{54}$ ) and $5 \mathrm{nM}$ pJES128 as template (containing the $K$. pneumoniae nifH-promoter regulatory region) (Berger et al., 1994). When analyzing the effect of the inhibitory activity of MBP-NifL synthesized under anaerobic and nitrogen limited conditions, all the reaction steps were performed under anaerobic conditions in the presence of $2 \mathrm{mM}$ dithiothreitol and inside an anaerobic chamber until open complex formation was completed. Subsequently, synthesis of transcripts was allowed by the addition of the nucleotide mix $(400 \mu \mathrm{M}$ ATP, $400 \mu \mathrm{M}$ GTP, $400 \mu \mathrm{M}$ UTP, $100 \mu \mathrm{M} \mathrm{CTP}, 200 \mathrm{kBq}$ $\left[\alpha-{ }^{32} \mathrm{P}\right]-\mathrm{CTP}, 0.1 \mathrm{mg} / \mathrm{ml}$ heparin) and further incubation for $10 \mathrm{~min}$ at $30{ }^{\circ} \mathrm{C}$ outside the anaerobic chamber. $\left(\alpha-{ }^{32} \mathrm{P}\right)$-CTP labeled transcripts were analyzed by electrophoresis in denaturing $6 \%$ polyacrylamide gels and quantified with a BAS 1500 Image Analyzer (Fuji) or with the PhospohorImager Storm (Molecular Dynamics).

Membrane preparations. Cytoplasmic and membrane fractions of cell-free cell extracts were separated by several centrifugation steps under aerobic or anaerobic conditions as recently described by Klopprogge et al. (Klopprogge et al., 2002) in the presence of the protease inhibitor cocktail for bacterial cell extracts (Sigma, Aldrich). The quality of the membrane preparations was evaluated by determination of the malate dehydrogenase activity in both the membrane and the cytoplasmic fraction, according to Bergmayer (Bergmayer, 1983). In addition quinoproteins were specifically detected by a redox-cycle stain assay to detect leakage of membrane proteins into the cytoplasmic fraction (Fluckiger et al., 1995). The MBP-NifL and GlnK bands of cytoplasmic and membrane fractions were quantified in Western blot analyses using known amounts of purified proteins as described above. Quantities of MBP-NifL and GlnK in the cytoplasmic and membrane fractions were calculated as relative to total MBP-NifL and GlnK, respectively, setting the absolute 
amounts of the respective protein in both fractions (cytoplasmic and membrane fraction) as $100 \%$.

\section{Results and Discussion}

We propose that GlnK transduces the nitrogen signal to the nif-regulatory system in $K$. pneumoniae by affecting the localization of NifL in response to the nitrogen status, possibly by direct interaction with NifL or the NifL/NifA complex. We thus examined (i) the formation of complexes between NifL, NifA and the primary nitrogen sensor GlnK and (ii) how GlnK effects NifL localization in response to the nitrogen status.

NifL and NifA form stoichiometric complexes after coexpression in $K$. pneumoniae. As no protein interactions between purified GlnK and MBP-NifL or MBP-NifA were detectable by co-chromotography on amylose resin, we decided to examine complex formation between the three regulatory proteins in vivo. MBP-fusion proteins of NifL and NifA expressed in $K$. pneumoniae have been shown to be functional and regulated normally in response to environmental changes (Berger et al., 1995; Narberhaus et al., 1995). Thus, we studied in vivo complex formation between NifL fused to the maltose binding protein (MBP-NifL) and a non-tagged NifA version by pull down experiments using affinity chromatography on amylose resin for detecting complexes. Synthesis of MBP-NifL and NifA was induced in K. pneumoniae under different nitrogen and oxygen availabilities to approximately equal amounts from the plasmid pRS201, which carries malE fused to the nifLA operon under the control of the tac promoter. Preparation of cell extracts and purification of MBP-NifL by affinity chromatography was performed under either aerobic or anaerobic conditions, respectively, in order not to change the oxygen conditions during cell breakage, fractionation and purification, which may effect the localization of MBP-NifL and / or the interaction between MBP-NifL and NifA. SDSPAGE analysis of the elution fractions showed that purification of MBP-NifL resulted in the isolation of MBP-NifL/NifA complexes, when synthesis occurred in the presence of oxygen under either nitrogen sufficiency $\left(+\mathrm{O}_{2}+\mathrm{N}\right)$ or limitation $\left(+\mathrm{O}_{2}-\mathrm{N}\right)$, or under anaerobic but nitrogen sufficient growth conditions $\left(-\mathrm{O}_{2}+\mathrm{N}\right)$. The amounts of NifL and NifA in those complexes were calculated by quantitative Western blot analysis using known amounts of purified proteins as standards, which were simultaneously quantified on 
the same blot as described in Materials and Methods (Fig. 2.1, lanes 1-6). Independent of the three different growth conditions, the absolute amounts of purified MBP-NifL/NifAcomplexes were comparable and the amount of NifA coeluting with MBP-NifL was in general within the range of $0.9 \pm 0.1$ NifA per MBP-NifL. Rechromatography further showed that up to $90 \%$ of the isolated complexes bound again to amylose resin, indicating that NifL/NifA-complexes formed in vivo are stable and do not rapidly dissociate upon storage at $4{ }^{\circ} \mathrm{C}$. These findings indicate that stable complexes between $K$. pneumoniae NifA and NifL are formed exclusively in vivo under physiological conditions, which is in contrast to A. vinelandii (Money et al., 1999; Money et al., 2001). Alternatively, for K. pneumoniae bridging proteins might be necessary for complex formation between NifL and NifA, which are missing in the in vitro analysis. However, we have not detected other proteins in significant amounts besides MBP-NifL and NifA in the complexes by silver staining.

In vivo complex formation between NifA and the cytoplasmic NifL fraction occurs independently of the nitrogen and oxygen status. Unexpectedly, significant but small amounts of MBP-NifL/NifA-complexes were also detected when synthesis occurred under nitrogen- and oxygen-limitation followed by purification of MBP-NifL under strictly anaerobic conditions (Fig. 2.1, lanes 7 to 11). The relative amount of these complexes was approx. $1 \%$ compared to the amounts of complexes seen with growth in the presence of either oxygen or ammonium or both; however the ratio between NifA and MBP-NifL was in the same range $(0.86 \pm 0.1$ NifA / MBP-NifL). As only MBP-NifL, not associated to membrane fragments, can be purified from cell extracts by affinity chromatography, this finding suggests that under simultaneous nitrogen- and oxygen-limitation only a small amount of MBP-NifL stays in the cytoplasm as has been shown for chromosomallyexpressed NifL (Klopprogge et al., 2002). This small amount of MBP-NifL remaining in the cytoplasm under derepressing conditions is apparently still able to interact and form inhibitory complexes with NifA in a stoichiometric 1:1 ratio (see Fig. 2.1, lanes 7 and 8); the majority of NifA, however, stays free in the cytoplasm and can activate nif gene transcription. In order to examine MBP-NifL localization in response to environmental signals we performed shift experiments. After synthesis of MBP-NifL and NifA under simultaneous nitrogen- and oxygen-limitation for $3 \mathrm{~h}$ in a 2-1 culture, the culture was split into three equal parts, one of which was further incubated for $30 \mathrm{~min}$; the other two were shifted to anaerobic growth in the presence of $10 \mathrm{mM}$ ammonium and aerobic nitrogen- 
limited growth for 30 min before cell harvest. Quantification of MBP-NifL in the different cell extract fractions separated under anaerobic or aerobic conditions respectively, showed that under derepressing conditions, approx. $95 \pm 3 \%$ of total MBP-NifL was found in the membrane-fraction in four independent experiments. However, after the shift to nitrogen or oxygen sufficiency, the relative amount of total MBP-NifL in the cytoplasmic fraction increased up to $88 \pm 8$ and $85 \pm 5 \%$, respectively. These data confirm that under derepressing conditions the majority of MBP-NifL is membrane-bound, the relative amount of NifA in the various cytoplasmic fractions, however, was nearly identical independently of the growth conditions.

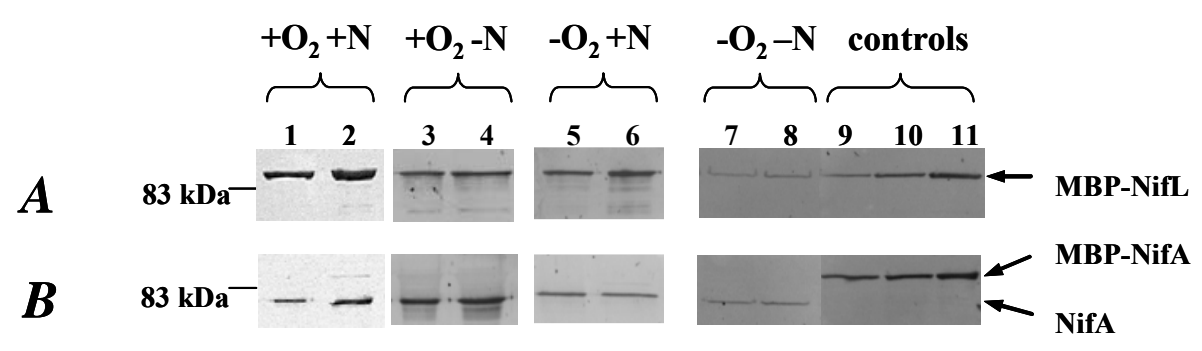

Figure 2.1: Coelution of MBP-NifL with NifA under various growth conditions after coexpression from pRS201 in $K$. pneumoniae. MBP-NifL was purified from cell extracts by affinity chromatography as described in Materials and Methods. The elution fractions 2 and 3, eluted in the presence of $10 \mathrm{mM}$ maltose in the buffer, were analyzed by SDS-PAGE and subsequent Western blot analysis using polyclonal antibodies raised against MBP-NifL (A) and MBP-NifA (B). Known amounts of purified MBP-NifL and MBP-NifA were simultaneously quantified on the same blot for each growth condition as exemplarily shown in lanes 9 to 11 for synthesis under derepressing conditions $\left(-\mathrm{O}_{2}-\mathrm{N}\right)$. Lanes 1 and 2, $5 \mu$ l elution fractions 2 and 3 after synthesis in the presence of oxygen and $10 \mathrm{mM}$ ammonium $\left(+\mathrm{O}_{2}+\mathrm{N}\right)$; lanes 3 and $4,5 \mu 1$ elution fractions after synthesis in the presence of oxygen and $4 \mathrm{mM}$ glutamine $\left(+\mathrm{O}_{2}-\mathrm{N}\right)$; lanes 5 and $6,5 \mu \mathrm{l}$ elution fractions after anaerobic synthesis in the presence $10 \mathrm{mM}$ ammonium $\left(-\mathrm{O}_{2}+\mathrm{N}\right)$; lanes 7 and $8,30 \mu \mathrm{l}$ elution fractions after synthesis under nitrogen and oxygen limitation $\left(-\mathrm{O}_{2}-\mathrm{N}\right)$; lanes 9 to $11,0.06,0.13$ and $0.25 \mu \mathrm{g}$ MBP-NifL respectively (A) and 0.06, 0.13 and $0.25 \mu \mathrm{g}$ MBP-NifA, respectively (B). Data are representative of four independent purifications for each growth condition. 
In order to obtain additional evidence that NifL remaining in the cytoplasm under derepressing conditions is still able to interact with NifA we characterized the inhibitory activity of anaerobically purified MBP-NifL synthesized under simultaneous nitrogen- and oxygen-limitation (MBP-NifL(-N,-O $\left.\mathrm{O}_{2}\right)$ ). Although in the absence of oxygen and ammonium, MBP-NifL(-N,- $\left.\mathrm{O}_{2}\right)$ in a purified in vitro transcription assay performed under anaerobic conditions clearly inhibited NifA transcriptional activity to approximately the same degree as aerobically synthesized and purified MBP-NifL in the presence of oxygen (Fig. 2.2). This indicates a direct protein-protein interaction between MBP-NifL(-N,- $\left.\mathrm{O}_{2}\right)$ and NifA, which is consistent with the finding of complex formation between cytoplasmic MBP-NifL and NifA under derepressing conditions. Based on those findings we conclude that in vivo complex formation between NifL and NifA in $K$. pneumoniae occurs independently of the nitrogen and oxygen status but is exclusively dependent on the localization of NifL in the cytoplasm.

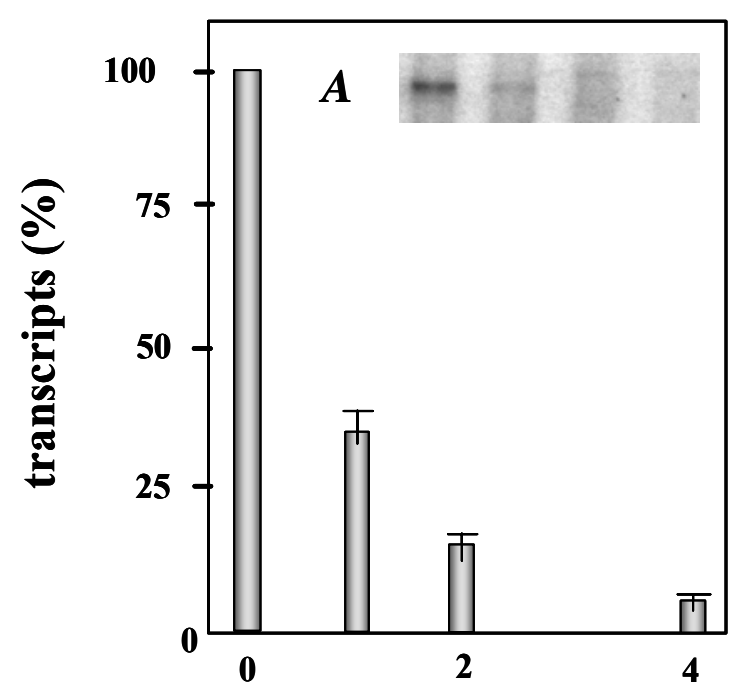

MBP-NifL $(\mu \mathrm{M})$

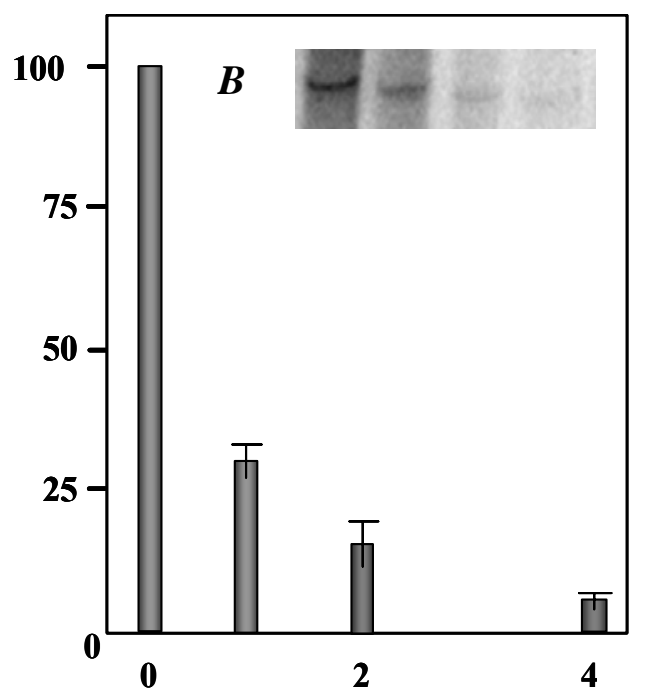

MBP-NifL(-N-O $)(\mu \mathrm{M})$

Figure 2.2: Effects of MBP-NifL synthesized under different conditions on transcriptional activation by the central domain of NifA. MBP-NifL was synthesized and purified (A) under aerobic and nitrogen sufficient conditions (MBP-NifL) or (B) under oxygen- and nitrogenlimitation (MBP-NifL(-N-O $\left.\mathrm{O}_{2}\right)$ ). Activities of the isolated central domain of NifA $(1 \mu \mathrm{M})$ were measured in the presence of different amounts of MBP-NifL in a single cycle transcription assay under aerobic (A) or anaerobic (B) conditions as described in Materials and Methods. Radioactivity in transcripts is plotted as a percentage of the maximum value (100\% NifA activity corresponded to approx. 11.2 fmol transcript). The presented data are based on at least 3 independent experiments; the insets show the corresponding radioactive transcription bands of one representative experiment for A and B in the presence of increasing inhibitor concentrations. 
Detection of a trimeric complex between NifA, NifL and GInK in $K$. pneumoniae. A regulatory role of GlnK in the modulation of NifA activity in response to the nitrogen status of the cell has previously been shown for several diazotrophic bacteria. GlnK protein appears to mediate the nitrogen status of the cell by direct protein-protein interaction with NifL in A. vinelandii (Little et al., 2002; Rudnick et al., 2002), and in diazotrophs, which do not contain NifL, there is evidence that GlnK or the paralogue GlnB-protein directly modulate the NifA activity in response to the nitrogen status (Arsene et al., 1996, 1999; Drepper et al., 2003; Liang et al., 1992). Thus, we further analyzed the elution fractions containing the MBP-NifL/NifA-complexes for the presence of chromosomally-expressed GlnK, using Western blot analysis. Interestingly, we could demonstrate the presence of small amounts of GlnK in the MBP-NifL/NifA-complexes purified from cells grown aerobically under nitrogen limitation for several independent experiments (Fig. 2.3A, left panel). Western blot analysis using antibodies raised against $\mathrm{GlnB}$ verified that it was GlnK which co-purified with the MBP-NifL/NifA-complex and not GlnB. In order to rule out that GlnK binds non-specifically to the MalE-fusion protein (MBP) or to the amylose resin itself, we coexpressed GlnK and MBP in K. pneumoniae from the plasmid pRS192, that contains both genes, malE and glnK, under the control of the tac promoter, and purified MBP by affinity chromatography. Western blot analysis showed that GlnK was not detectable in the elution fractions containing purified MBP, all synthesized GlnK was found in the flow through and wash fractions (data not shown). These findings strongly suggest that the chromosomally-synthesized GlnK protein detected within the purified MBP-NifL/NifA-complexes was pulled down from the cytoplasm and copurified with the MBP-NifL/NifA-complexes based on specific binding to either NifL or NifA, or to the NifL/NifA-complex.

In order to confirm the in vivo formation of a trimeric complex between NifL, NifA and GlnK, we coexpressed MBP-NifL, NifA and GlnK in K. pneumoniae under aerobic and nitrogen-limited growth conditions. Protein synthesis of approximately equivalent amounts of all three proteins was induced from plasmid pRS209, which contains the operon malE-nifLA and glnK under the control of the tac promoter. After purification, the complexes formed were analyzed by SDS-PAGE and silver staining, which showed that besides MBP-NifL, NifA and GlnK no other potentially bridging proteins were present in the elution fractions in significant amounts ( $>1 \%$ of GlnK amount). The ratio between the three regulatory proteins was determined from five independent purification experiments to be MBP-NifL:NifA:GlnK ${ }_{3}=1.0: 0.86 \pm 0.1: 0.16 \pm 0.015$ by quantitative Western blot 
analysis calculating GlnK concentrations as GlnK-trimers (Fig. 2.3A, right panel). These findings are the first indicating that in $K$. pneumoniae a NifL/NifA/GlnK-complex is formed during the transduction process of the nitrogen signal to the NifL/NifA-system by GlnK.

$\boldsymbol{A}$
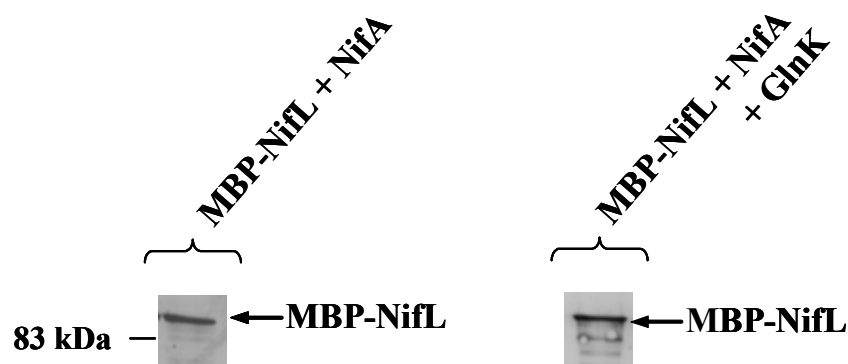

$83 \mathrm{kDa}-\stackrel{\mathrm{NifA}}{\longleftarrow}$

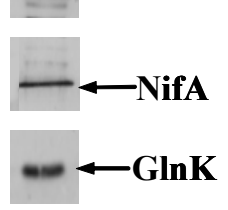

17 kDa

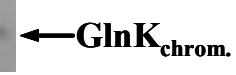

$\boldsymbol{B}$

$\begin{array}{lllll}1 & 2 & 3 & 4 & 5\end{array}$

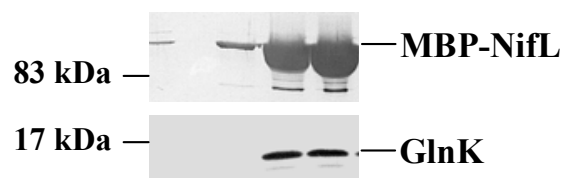

$\begin{array}{lllll}6 & 7 & 8 & 9 & 10\end{array}$

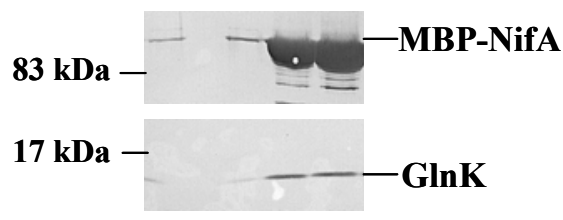

Figure 2.3: Coelution of GInK with NifL and NifA after coexpression in K. pneumoniae under nitrogen-limiting conditions. (A) MBP-NifL was purified from cell extracts by affinity chromatography as described in Materials and Methods. Aliquots of the purified MBP-NifL fractions were analyzed by SDS-PAGE and subsequent Western blot analysis using polyclonal antibodies raised against MBP-NifL, MBP-NifA or GlnK. For detecting NifL and NifA, $2 \mu 1$ aliquots were applied to the SDS-gel, and $20 \mu \mathrm{l}$ aliquots for detecting GlnK. Left panel, MBP-NifL coexpressed with NifA from pRS201 and chromosomally synthesized GlnK; right panel, MBPNifL coexpressed with NifA and GlnK from pRS209; data are representatives of three independent purifications. (B) After coexpression with GlnK under nitrogen-limiting growth conditions in K. pneumoniae, MBP-NifL and MBP-NifA were purified from cell extracts by affinity chromatography, respectively. $7.5 \mu \mathrm{l}$ of the elution fractions were analyzed by SDS-PAGE and subsequent Western blot analysis using polyclonal antibodies raised against NifL, NifA or GlnK as indicated. Left panel, MBP-NifL coexpressed with GlnK from pRS180: Lanes 1 and 2, wash fractions; lanes 3 - 5, elution fractions 1 - 3. Right panel, MBP-NifA coexpressed with GlnK from pRS158: Lanes 6 and 7, wash fractions; lanes $8-10$, elution fractions $1-3$. Data are representative of at least four independent purifications. 
The primary nitrogen-sensor protein GInK interacts simultaneously with both nif regulatory proteins, NifA and NifL. The finding that potentially a complex is formed between GlnK, MBP-NifL and NifA raises the question of whether GlnK interacts with NifL or NifA, or perhaps with both regulatory proteins during complex formation. In order to answer this question we coexpressed GlnK with MBP-NifL or MBP-NifA in K. pneumoniae to approximately equal amounts under aerobic and nitrogen-limited growth conditions from the plasmids pRS180 and pRS158, which both contain glnK and either malE-nifL or malE-nifA under the control of the tac promoter. The respective MBP-fusion proteins were purified by affinity chromatography and the elution fractions analyzed for coeluting GlnK. Interestingly, GlnK coeluted with both, MBP-NifL and MBP-NifA (Fig. 2.3B), indicating that GlnK interacts directly with both regulatory proteins as unspecific binding of GlnK to the affinity chromatography material and the MBP-fusion protein has been excluded. Quantification analysis of at least five independent purification experiments showed that approximately $0.2 \pm 0.02 \mathrm{GlnK}_{3}$ coeluted with MBP-NifL, which

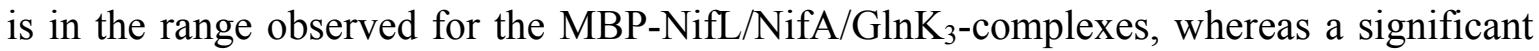
but lower ratio between $\mathrm{GlnK}_{3}$ and MBP-NifA was observed $\left(0.06 \pm 0.005 \mathrm{GlnK}_{3}\right.$ per MBP-NifA). This finding strongly indicates that under nitrogen limitation the primary nitrogen sensor GlnK interacts simultaneously with both regulatory proteins apparently transducing the signal of nitrogen limitation. The interaction between GlnK with NifL and NifA, however appeared to be weak as judged from the observed $\mathrm{GlnK}_{3}$ amount within the isolated complexes, potentially indicating that the GlnK-complexes are not stable.

GInK effects stability of NifL/NifA-complexes. In order to address the question, whether interaction with GlnK leads to dissociation of NifL/NifA-complexes we analyzed the effects of purified GlnK on isolated MBP-NifL/NifA-complexes preformed in vivo. Purified MBP-NifL/NifA-complexes (approx. 2 nmol) synthesized under ammonium and oxygen sufficiency were incubated at room temperature for $30 \mathrm{~min}$ in the presence of $4 \mathrm{nmol}$ purified GlnK in its unmodified state $\left(\mathrm{GlnK}_{3}\right)$ or completely uridylylated ((GlnK$\mathrm{UMP})_{3}$ ), or in the absence of GlnK. After repurification of MBP-NifL/NifA-complexes by affinity chromatography all fractions were analyzed for the presence of NifL, NifA and GlnK, the amounts of which were quantified by Western blot analysis. However, no complex dissociation was obtained in the presence of GlnK; MBP-NifL/NifA-complexes were purified to approximately the same amount $(1.9 \pm 0.1 \mathrm{nmol})$ and with approximately 
the same ratio between MBP-NifL and NifA (MBP-NifL : NifA = $1: 0.92 \pm 0.06$ ) independently of the GlnK presence.

As no effect of GlnK on NifL/NifA-complex stability was detectable in vitro, we examined the effect of additional GlnK synthesis on chromosomally (NtrC-dependent) expressed NifL and NifA in vivo. K. pneumoniae UN4495 containing glnK under the control of the tac promoter on a plasmid (pRS239) was grown under anaerobic conditions with $4 \mathrm{mM}$ glutamine as limiting nitrogen source and small amounts of ammonium. NtrC-independent synthesis of GlnK was induced with low IPTG concentrations $(0.1$ or $1.0 \mu \mathrm{M})$. Monitoring NifA-dependent transcription of the nifH'-'lacZ fusion during exponential growth showed that additional synthesis of $\mathrm{G} \ln \mathrm{K}$ in the absence of ammonium did not significantly influence nif induction, which was determined to be in the range of $2500 \pm$ $200 \mathrm{U} / \mathrm{ml} / \mathrm{OD}_{600}$ (Fig. 2.4A). In the presence of small amounts of ammonium, nif-induction was delayed independently of additional GlnK synthesis and started at approx. OD $_{600}=$ $0.37\left(0.25 \mathrm{mM} \mathrm{NH}_{4}{ }^{+}\right), \mathrm{OD}_{600}=0.6\left(0.5 \mathrm{mM} \mathrm{NH}_{4}{ }^{+}\right)$and $\mathrm{OD}_{600}=0.9\left(1.0 \mathrm{mM} \mathrm{NH}_{4}{ }^{+}\right)($Fig. 2.4B-D). This indicates that at those cell densities the respective amounts of ammonium were used up and NtrC-dependent synthesis of NifL and NifA occurred. However, compared to nitrogen limitation from the beginning (Fig. $2.4 \mathrm{~A}, \diamond$ ) the resulting nif induction in the absence of additional GlnK synthesis was significantly decreased in cultures initially containing small amounts of ammonium (Fig. 2.4B-D, $\diamond$ ). The Bgalactosidase synthesis was determined to be $1250 \pm 150 \mathrm{U} / \mathrm{ml}^{-} \mathrm{OD}_{600}\left(0.25 \mathrm{mM} \mathrm{NH}_{4}{ }^{+}\right.$cultures, Fig. 2.4B), $740 \pm 40 \mathrm{U} / \mathrm{ml} \mathrm{OD}_{600}$ (0.5 $\mathrm{mM} \mathrm{NH}_{4}{ }^{+}$-cultures, Fig. 2.4C), and $500 \pm$ $30 \mathrm{U} / \mathrm{ml} / \mathrm{OD}_{600}\left(1.0 \mathrm{mM} \mathrm{NH}_{4}{ }^{+}\right.$-cultures, Fig. 2.4D), indicating that NifL inhibition of NifA was not completely relieved. Additional GlnK synthesis in those cultures, however, restored nif induction to wild type levels under nitrogen limitation $(2500 \pm$ $200 \mathrm{U} / \mathrm{ml} / \mathrm{OD}_{600}$ ) (Fig. 2.4B-D, $\square, \triangle$ ). This finding indicates that either additional inhibitory NifL/NifA-complexes dissociated upon interaction with overexpressed GlnK or additional GlnK inhibited stable NifL/NifA-complex formation, both resulting in NifL sequestration at the cytoplasmic membrane and relief of NifA inhibition. 
A
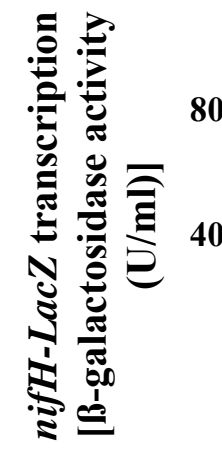

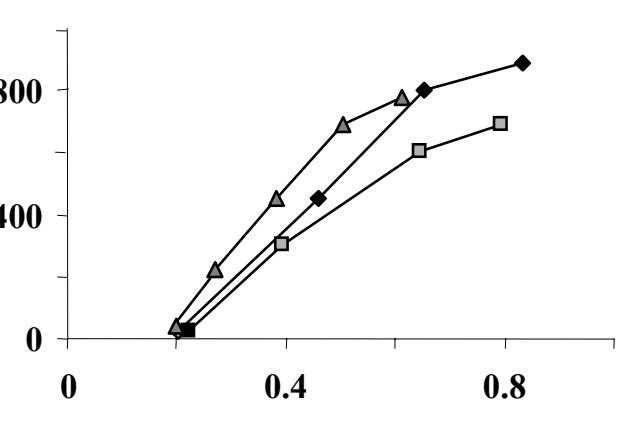

Optical density $\left(\mathrm{OD}_{600}\right)$

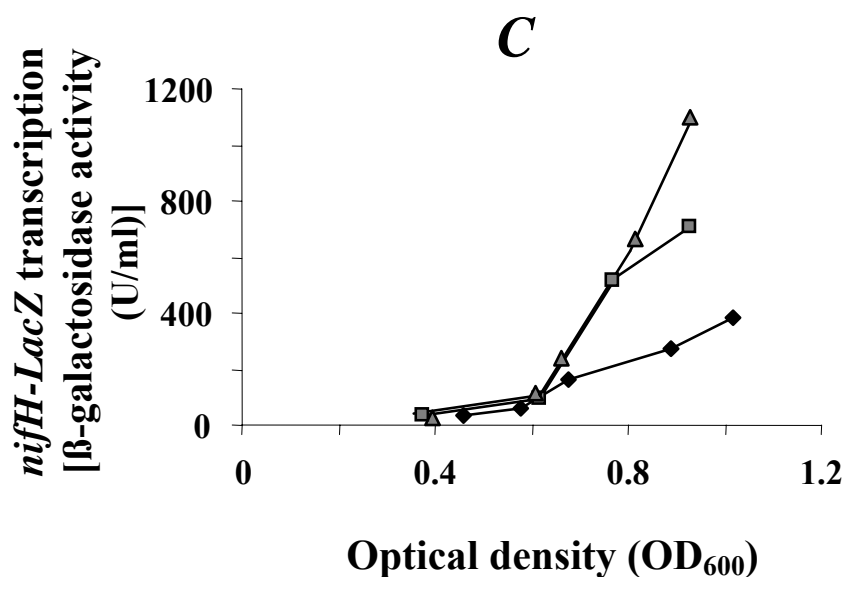

$\boldsymbol{B}$

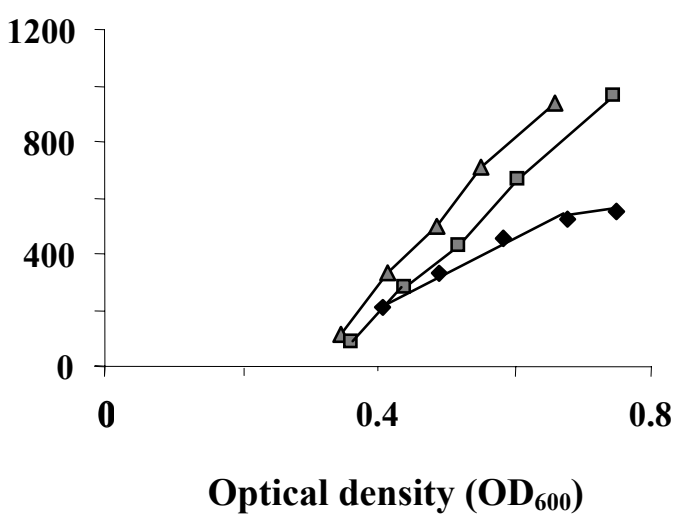

D

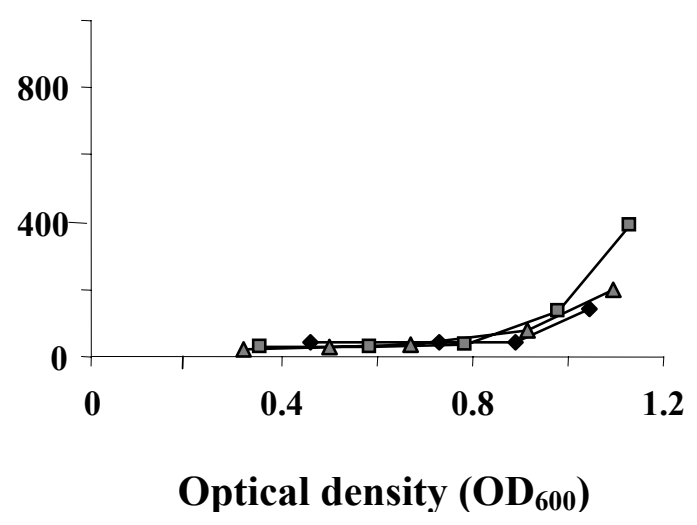

Figure 2.4: Effects of additional GlnK synthesis on nif induction in $K$. pneumoniae UN4495 in the presence of small amounts of ammonium. NifA-mediated activation of transcription from the nifHDK-promoter in K. pneumoniae UN4495 was monitored by measuring the B-galactosidase activity during anaerobic growth at $30^{\circ} \mathrm{C}$ in minimal medium with glutamine $(4 \mathrm{mM})$ as limiting nitrogen source (A) and with $4 \mathrm{mM}$ glutamine but in the presence of $0.25 \mathrm{mM}(\mathbf{B}), 0.5 \mathrm{mM}(\mathbf{C})$ and $1.0 \mathrm{mM}$ ammonium (D). NtrC-independent synthesis of GlnK was induced from plasmid pRS239 with 0.1 and $1.0 \mu \mathrm{M}$ IPTG. Activities of $\beta$-galactosidase were plotted as a function of $\mathrm{OD}_{600} .(\checkmark)$ UN4495; $(\triangle)$ UN4495/pRS239, $0.1 \mu \mathrm{M}$ IPTG; ( $\square$ ) UN4495/pRS239, $1.0 \mu \mathrm{M}$ IPTG. Data are representative of three independent growth experiments.

In order to obtain further evidence we analyzed, whether additional synthesis of GlnK effects complex formation between NifL and NifA under oxygen limitation and nitrogen sufficiency. Under those growth conditions, significant amounts of MBP-NifL/NifAcomplexes were isolated, when MBP-NifL and NifA synthesis occurred from plasmid pRS201, in the absence of additional GlnK synthesis (see Fig. 2.1, lanes 5 and 6). 


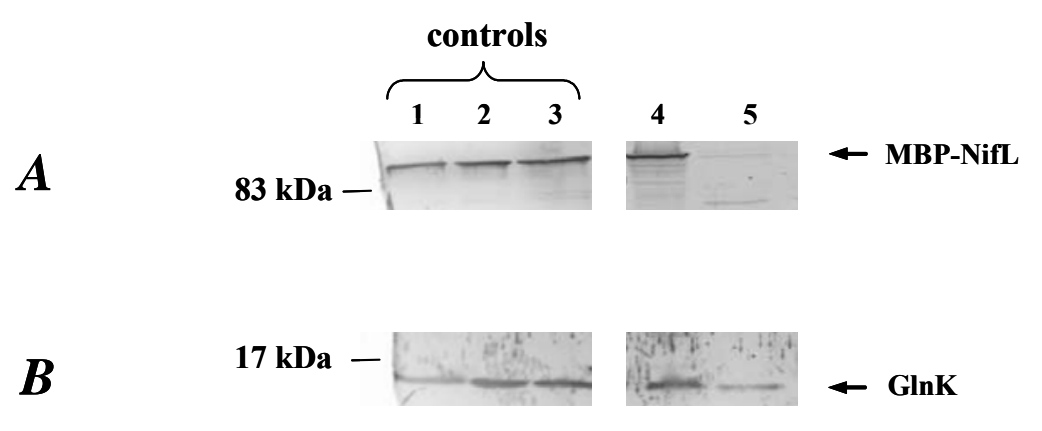

Figure. 2.5: Localization analysis of MBP-NifL synthesized under anaerobic and nitrogen sufficient conditions in the presence of NtrC-independent GInK synthesis. MBP-NifL, NifA and GlnK were synthesized from plasmid pRS209 with $100 \mu \mathrm{M}$ IPTG under anaerobic conditions but in the presence of $10 \mathrm{mM}$ ammonium at $30{ }^{\circ} \mathrm{C}$. Cell extract was prepared and separated into membrane and cytoplasmic fractions as described in Materials and Methods. Aliquots of the observed membrane and cytoplasmic fraction were subjected to SDS-PAGE, and subsequently analyzed by Western blotting. Polyclonal antibodies directed against MBP-NifL (A) or GlnK (B) were used to detect MBP-NifL and GlnK in the different fractions and protein amounts were quantified with a fluoroimager (Molecular Dynamics) using purified proteins as described in Material and Methods. Quantities of NifL and GlnK in the cytoplasmic and membrane fractions were calculated as relative to total NifL and total GlnK, respectively, setting the absolute amounts in both fractions (cytoplasmic and membrane fraction) of the respective protein as $100 \%$. Lanes 1 to 3, controls for quantification, 0.03, 0.065 and $0.13 \mu \mathrm{g}$ MBP-NifL (A) and 0.028, 0.056 and $0.113 \mu \mathrm{g} \mathrm{GlnK}(\mathbf{B})$; lane $4,4 \mu \mathrm{l}$ of the membrane fraction $(0.9 \mathrm{ml})$; lane $5,4 \mu \mathrm{l}$ of the cytoplasmic fraction $(4.2 \mathrm{ml})$. Data are representative of four independent membrane preparations.

However, when GlnK was additionally synthesized under oxygen limitation in the presence of $10 \mathrm{mM}$ ammonium, using plasmid pRS209 for NtrC-independent synthesis of MBP-NifL, NifA and GlnK, the purification under anaerobic conditions did not result at all in the isolation of MBP-NifL or a complex including MBP-NifL. Localization analysis of MBP-NifL in those cells further showed that $95 \pm 2 \%$ of total MBP-NifL was found in the membrane fraction (Fig. 2.5), which is consistent with the finding that no MBP-NifL was purified from the soluble cell extract. Interestingly, $70 \pm 5 \%$ of total GlnK was also found in the membrane fraction. However, at the current experimental status we do not know, whether the overproduced GlnK binds to the cytoplasmic membrane in a NifL-dependent manner. The relative amounts of NifA in the cytoplasm did not change upon additional GlnK synthesis. These findings, which were confirmed by several independent experiments, again strongly indicate that the additional GlnK synthesis resulted in the dissociation of the inhibitory NifL/NifA complexes or inhibited the formation of stable 
NifL/NifA-complexes. Thus, we conclude that GlnK effects the cellular localization of NifL in response to the nitrogen status by influencing the formation of NifL/NifAcomplexes. This proposed mechanism for nitrogen signal transduction by GlnK in K. pneumoniae differs significantly from the mechanism of nitrogen signal transduction by GlnK in A. vinelandii (Little et al., 2000; Little et al., 2002; Little and Dixon, 2003; Rudnick et al., 2002).

\section{Hypothetical model for oxygen and nitrogen control of nif regulation in}

K. pneumoniae. On the basis of those data and the finding that only small amounts of $\mathrm{GlnK}_{3}$ are present in the MBP-NifL/NifA/GlnK-complexes formed under nitrogenlimitation, we hypothesize that the NifL/NifA/GlnK-complex reflects a transitional status within the signal transduction of nitrogen-limitation to the NifL/NifA system and propose the following working model (Fig. 2.6). Under anaerobic and nitrogen-limited conditions, the interaction with GlnK eventually results in unbound NifA and NifL, which is able to receive electrons at the cytoplasmic membrane from the anaerobic quinol pool (Grabbe and Schmitz, 2003). Upon reduction NifL is sequestered to the cytoplasmic membrane and thus allows NifA to activate nif genes in the cytoplasm (Fig. 2.6A). After a period of oxygenand nitrogen-limitation, an ammonium-upshift results in deuridylylation of GlnK and unmodified GlnK may be sequestered to the cytoplasmic membrane in an AmtB-dependent manner as has been recently shown for E. coli and A. vinelandii GlnK (Coutts et al., 2002). Sequestration of GlnK to the cytoplasmic membrane would significantly reduce NifA/NifL-complex dissociation by GlnK; consequently, most of NifL stays in the cytoplasm as recently demonstrated (Klopprogge et al., 2002) and inhibits NifA activity by forming inhibitory complexes (Fig. 2.6B). When a shift to oxygen occurs in addition, NifL is oxidized and upon oxidation the main part of NifL dissociates from the cytoplasmic membrane and forms inhibitory NifL/NifA-complexes in the cytoplasm (Fig. 2.6C and D). This occurs even under nitrogen-limitation in the presence of GlnK, as membrane-bound reduced NifL is rapidly oxidized and quickly dissociates into the cytoplasm resulting in a high NifL/NifA-complex formation rate, which appears to be much higher than the GlnKdependent dissociation rate. 


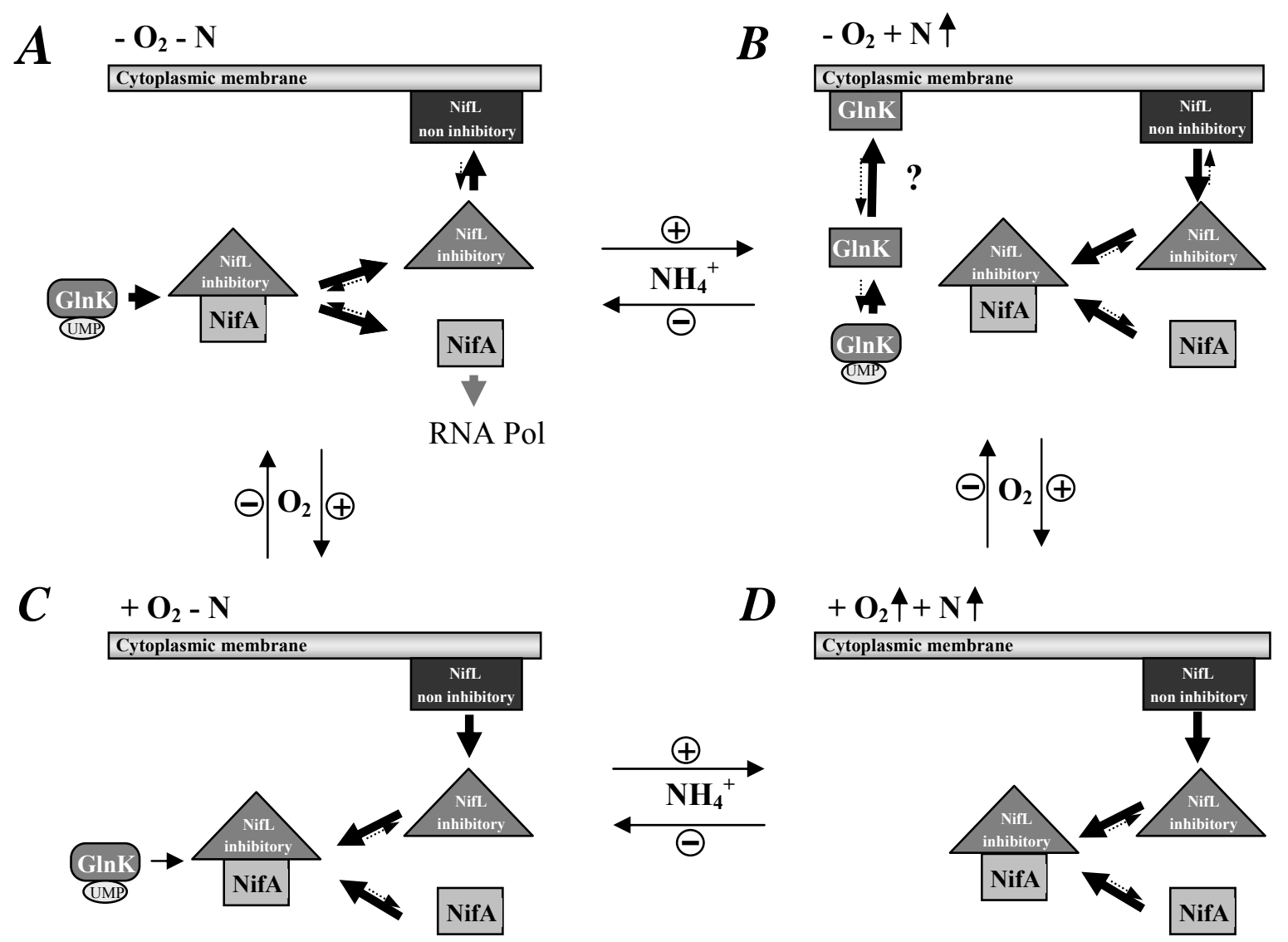

Figure 2.6: Hypothetical regulation model. The regulatory mechanism is primarily based on changes in the cellular localization of regulatory proteins in response to changes in environmental signals. (A) Simultaneous nitrogen- and oxygen limitation $\left(-\mathrm{O}_{2}-\mathrm{N}\right),($ B) oxygen limitation but shift to nitrogen sufficiency $\left(-\mathrm{O}_{2}+\mathrm{N}\right),(\mathbf{C})$ aerobic but nitrogen limiting growth conditions $\left(+\mathrm{O}_{2}-\mathrm{N}\right)$, and (D) simultaneous aerobic and nitrogen sufficient growth conditions $\left(+\mathrm{O}_{2}+\mathrm{N}\right)$.

Acknowledgements. J.S. and R.T. contributed equally to this work and should both be considered first authors. We thank Gerhard Gottschalk for generous support, helpful discussions, and lab space, and Andrea Shauger for critical reading the manuscript. This work was supported by the Deutsche Forschungsgemeinschaft (SCHM1052/4-4 and 4-5) and the Fonds der Chemischen Industrie. 
Chapter 3:

\section{Biochemical studies of Klebsiella pneumoniae NifL reduction using reconstituted partial anaerobic respiratory chains of Wolinella succinogenes}

\section{Summary}

In the diazotroph Klebsiella pneumoniae the flavoprotein NifL inhibits the activity of the nif specific transcriptional activator NifA in response to molecular oxygen and combined nitrogen. Sequestration of reduced NifL to the cytoplasmic membrane under anaerobic and nitrogen-limited conditions impairs inhibition of cytoplasmic NifA by NifL. In order to analyze whether NifL is reduced by electrons directly derived from the reduced menaquinone pool we studied NifL reduction using artificial membrane systems containing purified components of the anaerobic respiratory chain of Wolinella succinogenes. In this in vitro assay using proteoliposomes containing purified formate dehydrogenase and purified menaquinone $\left(\mathrm{MK}_{6}\right)$ or 8-methyl-menaquinone $\left(\mathrm{MMK}_{6}\right)$ from $W$. succinogenes, reduction of purified NifL was achieved by formate oxidation. Further the respective reduction rates, which were determined using equal amounts of NifL, have been shown to be directly dependent on the concentration of both, formate dehydrogenase and menaquinones incorporated into the proteoliposomes, demonstrating a direct electron transfer from menaquinone to NifL. When purified hydrogenase and $\mathrm{MK}_{6}$ from $W$. succinogenes were inserted into the proteoliposomes NifL was reduced with nearly the same rate by hydrogen oxidation. In both cases, reduced NifL was found to be highly associated to the proteoliposomes, which is in accordance with our previous findings in vivo. On the bases of these experiments, we propose that the redox state of the menaquinone pool is the redox signal for nif-regulation in K. pneumoniae by directly transferring electrons onto NifL under anaerobic conditions. 


\section{Introduction}

In the free living nitrogen fixing K. pneumoniae transcription of the nitrogen fixation (nif)genes is strictly regulated in response to external oxygen and combined nitrogen. Under nitrogen- and oxygen-limiting conditions, transcription of nif-genes is mediated by the activator protein NifA in combination with the alternative $\sigma^{54}$-RNA polymerase (Hoover et al., 1990; Morett and Buck, 1989; Ray et al., 2002). However, in the presence of molecular oxygen and / or ammonium NifA transcriptional activity is inhibited by a second regulatory protein, NifL (Egener et al., 2002; Hill et al., 1981; Hill et al., 1996; Merrick, 1982). This inhibition of NifA activity by NifL occurs via direct protein-protein interaction in K. pneumoniae, which was first implied by co-immunoprecipitation studies (Henderson et al., 1989) and has been recently demonstrated by pull-down experiments (Stips et al., 2004). A direct protein interaction between NifL and NifA is further consistent with yeasttwo-hybrid studies and in vitro analysis of complex formation between NifL and NifA from $A$. vinelandii, which also regulates its nif-gene expression by the two regulatory proteins (Lei et al., 1999; Little et al., 2000; Money et al., 1999; Money et al., 2001; Reyes-Ramirez et al., 2002).

The negative regulator NifL, which modulates NifA activity in response to the oxygen and nitrogen status of the cell, is a flavoprotein (Egener et al., 2002; Hill et al., 1996; Macheroux et al., 1998; Schmitz, 1997) reviewed in (Little et al., 2006; Martinez-Argudo et al., 2004c, 2005; Schmitz et al., 2002). By analyzing in vivo complex formation between NifA and NifL, we have recently demonstrated that sequestration of the inhibitor NifL to the cytoplasmic membrane under nitrogen and simultaneous oxygen limitation (derepressing conditions) is the key mechanism for regulating cytoplasmic NifA activity in K. pneumoniae by NifL (Klopprogge et al., 2002; Stips et al., 2004) (see Fig. 3.1). We further showed that NifL sequestration to the cytoplasmic membrane is directly dependent on the reduction of its FAD-cofactor under anaerobic and nitrogen limitation conditions (Grabbe and Schmitz, 2003; Klopprogge et al., 2002; Stips et al., 2004).

The cellular nitrogen status is transduced towards the NifL/NifA regulatory system by direct protein interaction with the nitrogen sensory protein $\mathrm{GlnK}$ in both, K. pneumoniae and A. vinelandii, (Arcondeguy et al., 1999; Arcondeguy et al., 2000; He et al., 1998; Jack et al., 1999; Little et al., 2000; Reyes-Ramirez et al., 2001; Stips et al., 2004). In A. vinelandii, unmodified GlnK activates the inhibitory function of NifL under nitrogen excess by direct protein interaction (Little et al., 2002; Rudnick et al., 2002); whereas 
under nitrogen limitation, the inhibitory activity of $A$. vinelandii NifL appears to be relieved by elevated levels of 2-oxoglutarate (Little et al., 2000; Little et al., 2002; Little and Dixon, 2003; Martinez-Argudo et al., 2004b; Perry et al., 2005; Reyes-Ramirez et al., 2001; Rudnick et al., 2002). In contrast to A. vinelandii, the relief of NifL inhibition in K. pneumoniae under nitrogen limitation depends on the interaction with modified GlnK (Arcondeguy et al., 1999; Arcondeguy et al., 2000; He et al., 1998; Jack et al., 1999). We have recently shown that GlnK effects the cellular localization of NifL in response to nitrogen limitation by direct protein interaction with the inhibitory NifL/NifA-complex resulting in complex dissociation (Stips et al., 2004) followed by NifL sequestration to the cytoplasmic membrane when oxygen is simultaneously limiting (Klopprogge et al., 2002) (see Fig. 3.1).

Recent genetic studies strongly suggest that the electrons, which are responsible for FAD reduction, are derived from the anaerobic respiratory chain of $K$. pneumoniae via the reduced quinone pool (Grabbe et al., 2001; Grabbe and Schmitz, 2003). In order to obtain further evidence we established an in vitro system to reduce purified NifL using proteoliposomes containing purified components of the anaerobic respiratory chain of the anaerobic rumen bacterium Wolinella succinogenes (formate dehydrogenase, hydrogenase and menaquinones) (Dietrich and Klimmek, 2002). Studying NifL reduction using this artificial membrane system and the respective electron donors (formate, hydrogen) we achieved strong evidence (i) that under anaerobic conditions electrons are directly transferred from the reduced menaquinone pool in the membrane onto NifL-bound FAD; and (ii) that the requirement for any further K. pneumoniae specific protein potentially interacting with NifL - such as a specific oxidoreductase or a NifL-specific receptor protein in the cytoplasmic membrane - can be excluded. 


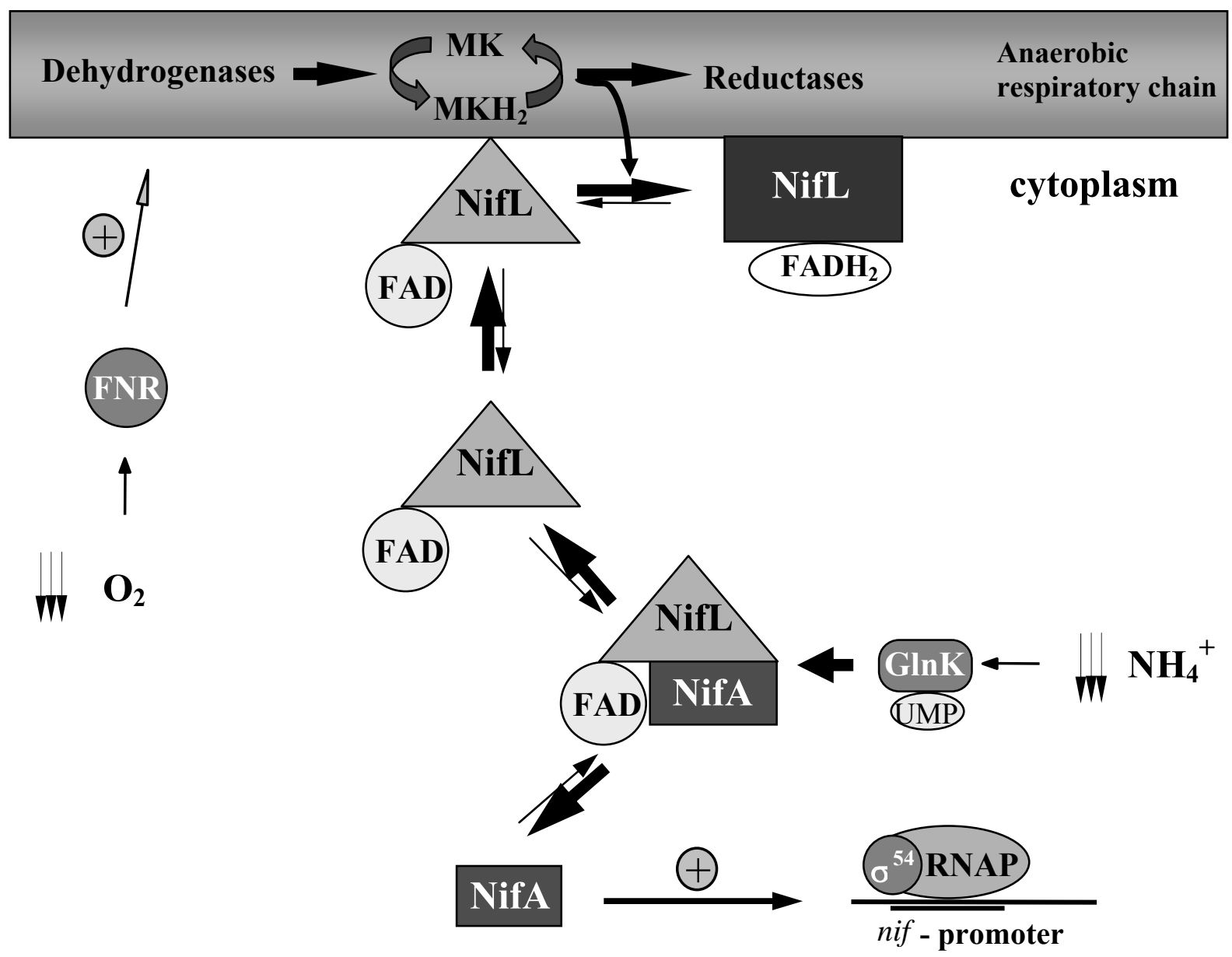

Figure. 3.1: Working model for oxygen and nitrogen signal transduction in K. pneumoniae. Sequestration of cytoplasmic NifL to the cytoplasmic membrane under simultaneous nitrogen and oxygen limiting conditions is the key mechanism of regulating cytoplasmic NifA activity. Interaction with modified GlnK in response to nitrogen limitation results in complex dissociation of the inhibitory NifL/NifA complex (Stips et al., 2004), followed by NifL reduction at the cytoplasmic membrane by electrons derived from the reduced menaquinone-pool (Grabbe et al., 2001; Grabbe and Schmitz, 2003)

\section{Experimental procedures}

Bacterial Strains. The bacterial strain used in this work was K. pneumoniae M5al (wild type) containing pJES794 (Narberhaus et al., 1995), which carries malE-nifL under the control of the tac promoter.

Purification and reconstitution of the FAD-cofactor of MBP-NifL. MBP-NifL was synthesized at $30^{\circ} \mathrm{C}$ in maximal induction medium (Ow and Ausubel, 1983) in K. pneumoniae carrying pJES794 (Narberhaus et al., 1995). Expression of fusion protein 
was induced from the tac promoter for $4 \mathrm{~h}$ with $100 \mu \mathrm{M}$ isopropyl- $\beta-\mathrm{D}$-thiogalactopyranoside (IPTG) at $19{ }^{\circ} \mathrm{C}$ when cultures reached a turbidity of O.D. $600=0.6$. After disruption of cells in breakage (B) buffer and centrifugation at $20.000 \mathrm{x} \mathrm{g}$, fusion proteins were purified from the supernatant by amylose affinity chromatography (Schmitz, 1997). In order to reconstitute the fraction of apo-flavoprotein, the method was modified in a way that a solution of $10 \mathrm{mM}$ FAD in B-buffer was added to matrix immobilized MBP-NifL and circulated over the column for $15 \mathrm{~h}$ at $4{ }^{\circ} \mathrm{C}$ (Fig. 3.2A) prior to the elution of MBPNifL.

To determine the molar extinction coefficient of NifL-bound FAD at least three independent MBP-NifL purifications were analyzed. Initially the red-ox spectra of these samples were monitored and the differences of absorbance at $452 \mathrm{~nm}$ were determined $\left(\Delta \mathrm{E}_{452-600 \mathrm{~nm}}\right)$ for each sample. Subsequently, the FAD-cofactor of the respective MBP-NifL fractions was extracted with TCA as recently described (Schmitz, 1997) and the amounts of extracted FAD were calculated by fluorescent quantification (excitation at $465 \mathrm{~nm}$ and monitoring emission at $520 \mathrm{~nm}$ ) using FAD-solutions in B-buffer containing $5 \%$ TCA as standards. The absorbance difference $\left(\Delta \mathrm{E}_{452-600 \mathrm{~nm}}\right)$ obtained from the red-ox spectra and the respective amount of extracted FAD were used to calculate the molar extinction coefficient which was determined to be $\varepsilon_{452 \mathrm{~nm}-600 \mathrm{~nm}}=6.63 \mathrm{mM}^{-1} \mathrm{x} \mathrm{cm}^{-1}$. Using this molar extinction coefficient, the FAD content of purified MBP-NifL fractions was routinely calculated from their respective red-ox spectra (Fig. 3.2B). Reconstituted MBP-NifL contained between 0.9 and 1.0 mol FAD-cofactor per mol MBP-NifL. In general, only fully reconstituted MBPNifL protein charges ( $1 \mathrm{~mol} \mathrm{FAD} / \mathrm{mol} \mathrm{MBP}-\mathrm{NifL})$ were used in the following reduction assays.

Purifiaction of formate dehydrogenase, hydrogenase and menaquinones of $W$. succinogenes. Formate dehydrogenase and hydrogenase of $W$. succinogenes were isolated as described previously (Dross et al., 1992; Kröger, 1994; Unden and Kroger, 1986). The quinones of $W$. succinogenes menaquinone ${ }_{6}\left(\mathrm{MK}_{6}\right)$ and 8-methylmenaquinone $_{6}\left(\mathrm{MMK}_{6}\right)$ were extracted from the membrane fraction using a mixture of petrol ether and methanol $(70 / 30 \mathrm{v} / \mathrm{v})$. The different quinones in the extract were separated by HPLC according to Unden (Unden, 1988). The separated quinones were quantified by HPLC using vitamin $\mathrm{K}_{1}$ (Fluka) as a standard (Dietrich and Klimmek, 2002). 
Preparation of proteoliposomes. Sonic liposomes containing menaquinones from $W$. succinogenes were prepared from a mixture of egg phospholipids $(95 \%$, w/w) (Singleton et al., 1965) and phosphatidylethanolamine (5\%, Fluka No 60650) as described (Unden and Kröger, 1982). Proteoliposomes were prepared by freeze-thawing sonic liposomes containing the quinones indicated and either hydrogenase or formate dehydrogenase as described by Unden and Kröger (Unden and Kroger, 1986).

Reduction of MBP-NifL by formate oxidation or hydrogen oxidation using artificial membrane systems. All analyses of MBP-NifL reduction were performed in the absence of oxygen under strictly anaerobic conditions in volume reduced glass cuvettes. The reduction of MBP-NifL by formate (or hydrogen respectively) was recorded as the absorbance difference at $452 \mathrm{~nm}$ minus $600 \mathrm{~nm}\left(\Delta \varepsilon_{452 \mathrm{~nm}-600 \mathrm{~nm}}=6.63 \mathrm{mM}^{-1} \mathrm{x} \mathrm{cm}^{-1}\right)$ in B-buffer containing a constant amount of $4.2 \mathrm{nmol}$ MBP-NifL in a total test volume of $400 \mu \mathrm{l}$ and solubilised formate dehydrogenase (or hydrogenase respectively) or in proteoliposomal membranes ( $5 \mathrm{mg}$ phospholipid in $400 \mu$ l test volume) integrated formate dehydrogenase with amounts indicated in Table 1. The additional electron mediator DMN or MK respectively in proteoliposomal membranes were also added as indicated in Table 1. The red-ox reaction was started by the addition of an initial concentration of $12.5 \mathrm{mM}$ formate (or by the addition of molecular hydrogen). The reduction of MBP-NifL was archived by recording spectra from $600 \mathrm{~nm}$ to $360 \mathrm{~nm}$ in time intervals using a spectral photometer (Spektrophotometer U-3000, Hitachi). The difference in absorbance at $452 \mathrm{~nm}$ corrected for the difference in absorbance at $600 \mathrm{~nm}$ (turbidity and additives) of the resulting red-ox spectra was used to calculate the amount of MBP-NifL reduction rate by time using the extinction coefficient of MBP-NifL bound FAD determined earlier $\left(\Delta \varepsilon_{452 \mathrm{~nm}-600 \mathrm{~nm}}=6.63 \mathrm{mM}^{-1} \mathrm{x} \mathrm{cm}^{-1}\right)$.

Analyzing membrane association of reduced MBP-NifL by sucrose gradient centrifugation. In a test volume of $250 \mu 16.1 \mathrm{nmol}$ MBP-NifL were fully reduced by an excess of formate (15 mM) using proteoliposomes (6.25 $\mathrm{mg}$ phospholipids) containing purified formate dehydrogenase $(2.5 \mathrm{nmol}$, i.e. $64 \mu \mathrm{g} / \mathrm{mg}$ phospholipid $)$ and menaquinone ${ }_{6}(18 \mathrm{nmol} / \mathrm{mg}$ phosholipid) from W. succinogenes (Dietrich and Klimmek, 2002). The complete assay was subsequently subjected under anaerobic conditions to an anaerobic sucrose gradient (10 - $40 \%$ sucrose in B-buffer complemented with $15 \mathrm{mM}$ formate by a total volume of $28 \mathrm{ml}$ ). After ultracentrifugation at $175.000 \mathrm{x} \mathrm{g}$ for $270 \mathrm{~min}$ formate dehydrogenase 
activity of the different fractions were assayed as described recently (Biel et al., 2002); and the various fractions were analyzed for NifL presence and by Western blot analysis. For ultracentrifugation experiments in the presence of oxygen, the presence of formate dehydrogenase in the fractions was analysed by Western blot analysis. Fractions were separated on denaturating polyacrylamide gels and transferred to nitrocellulose membranes (BioTrace ${ }^{\circledR}$ NT, Pall Life Science) (Sambrook, 1989). Membranes were subsequently exposed to specific polyclonal rabbit antisera directed against MBP-NifL of K. pneumoniae or formate dehydrogenase of $W$. succinogenes. Protein bands were detected with secondary antibodies directed against rabbit immunoglobulin $G$ and coupled to horseradish peroxidase (BioRad Laboratories), visualized and quantified using the ECLplus system (Amersham /Pharmacia) with a fluoroimager (DianaIII, Raytest).

\section{Results}

The goal of this work was to gain a deeper insight into the NifL reduction in K. pneumoniae resulting in membrane sequestration of the negative regulator under simultaneous nitrogen and oxygen limiting conditions. In order to address the question, whether electrons are directly transferred from the reduced quinone pool onto the flavoprotein NifL or via a NifL specific protein (reductase or receptor), we studied NifL reduction using a partial reconstituted anaerobic respiratory chain from $W$. succinogenes.

Purification of fully reconstituted NifL-protein and components of the W. succinogenes anaerobic respiratory chain. Following MBP-NifL synthesis in K. pneumoniae under low temperatures $\left(19^{\circ} \mathrm{C}\right)$ reconstitution of the $\mathrm{FAD}$-cofactor into the flavoprotein was achieved by continuous circulation of a $10 \mathrm{mM}$ FAD solution over matrix-immobilized MBP-NifL at $4{ }^{\circ} \mathrm{C}$ (see Fig. 3.2 and Experimental Procedures). The resulting purified MBP-NifL fractions, which in general contained approximately one mol FAD-cofactor per mol MBPNifL, were used in the in vitro reduction assays described below. The respective molar extinction coefficient at $452 \mathrm{~nm}$ for NifL-bound FAD was determined to be $\Delta \varepsilon_{452 \mathrm{~nm}-600 \mathrm{~nm}}=$ $6.63 \mathrm{mM}^{-1} \mathrm{x} \mathrm{cm}^{-1}$ as described in Experimental Procedures. For the reconstitution of partial anaerobic respiratory chains in liposomes native formate dehydrogenase, native hydrogenase and both menaquinones (menaquinone ${ }_{6}\left(\mathrm{MK}_{6}\right)$ and 8 -methyl-menaquinone 6 
$\left.\left(\mathrm{MMK}_{6}\right)\right)$ were purified from membranes of $W$. succinogenes as described by Dietrich and Klimmek (Dietrich and Klimmek, 2002) and Biel et al. (Biel et al., 2002).

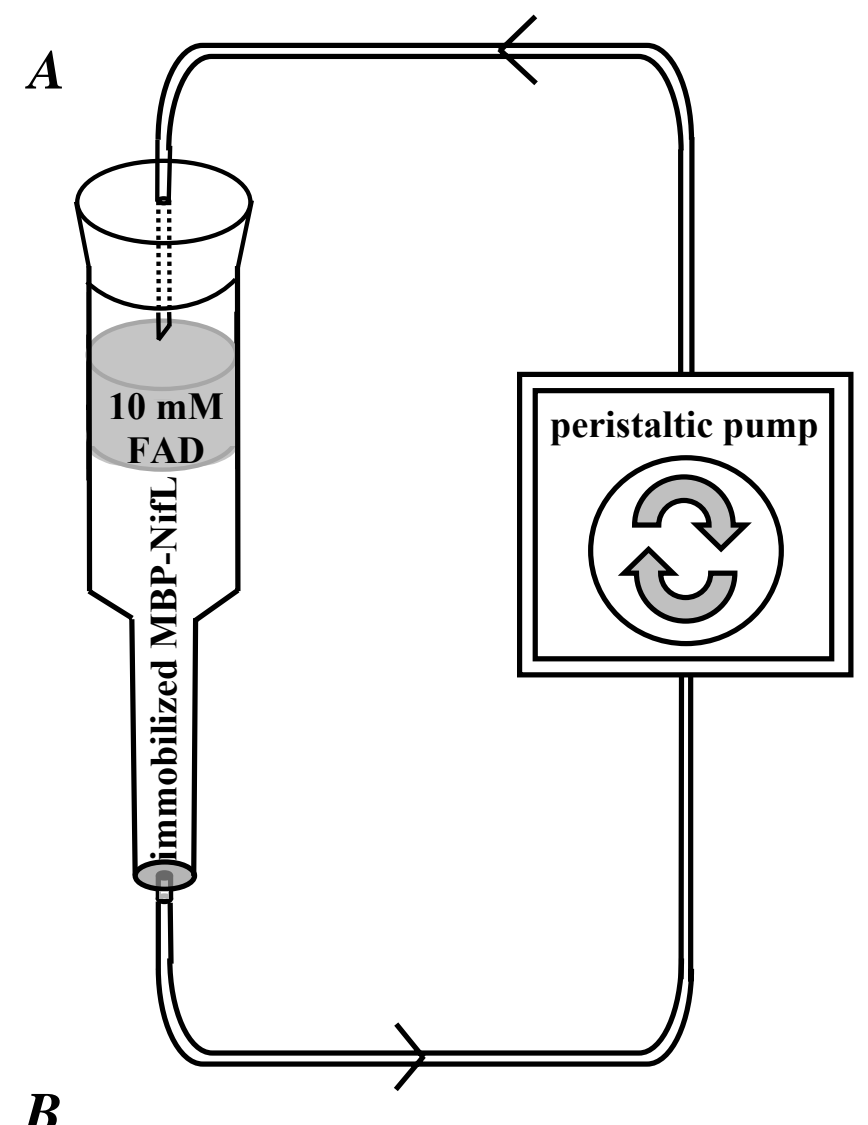

$\boldsymbol{B}$

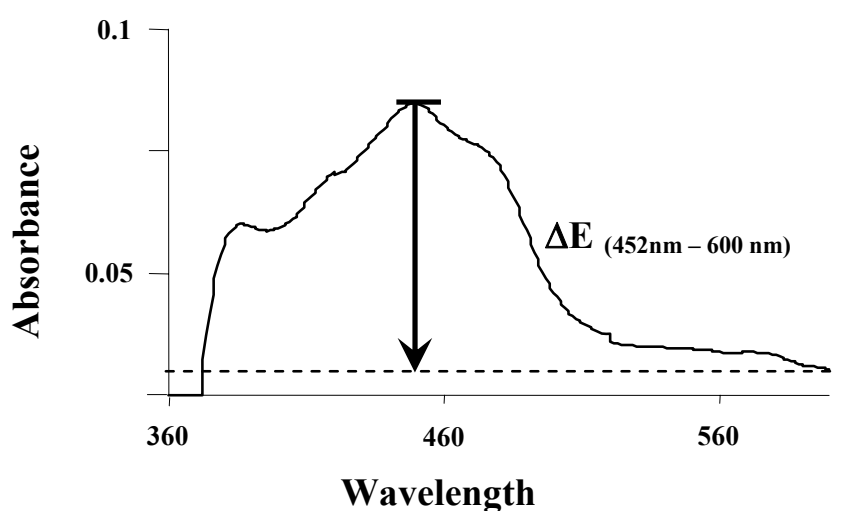

Figure 3.2: Reconstitution of immobilized MBP-NifL with FAD. MBP-NifL was expressed in K. pneumoniae and purified as described in Experimental Procedures. A) MBP-NifL of the cell extracts was immobilized to the affinity chromatography matrix; subsequently a $10 \mathrm{mM}$ FAD solution was added and circulated over the column for 15 hours at $4{ }^{\circ} \mathrm{C}$ yielding in a $100 \%$ reconstitution of the FAD-cofactor of MBP-NifL. B) UV-vis difference spectrum (oxidized reduced) of fully reconstituted MBP-NifL $(9.1 \mu \mathrm{M})$, oxidized with molecular oxygen and reduced with $5 \mathrm{mM}$ dithionite. 
Reduction of MBP-NifL by formate oxidation catalyzed by solubilised membranous formate dehydrogenase of $W$. succinogenes. All the following analyses of MBP-NifL reduction were performed in the absence of oxygen under strictly anaerobic conditions in a test volume of $400 \mu \mathrm{l}$ (see Experimental Procedures). As an initial control formate oxidation by purified solubilised formate dehydrogenase from membranes of the anaerobic rumen bacterium $W$. succinogenes was studied, which demonstrated that cytochrome $b$ of $W$. succinogenes formate dehydrogenase $(34 \mu \mathrm{g})$ was fully reduced in less than 1 min after adding $12.5 \mathrm{mM}$ formate (data not shown). Thus, in the following test assays $12.5 \mathrm{mM}$ formate was routinely added as electron donor. In order to analyze, whether electrons derived from formate oxidation can in principle be transferred to and reduce MBP-NifL in solution, formate was oxidized by purified solubilised $W$. succinogenes formate dehydrogenase $(34 \mu \mathrm{g})$ in the presence of $4.2 \mathrm{nmol}$ purified MBP-NifL in the test assay. In the presence of $0.2 \mathrm{mM}$ of the water soluble quinone dimethylnaphtoquinone (DMN), which is reduced by the cytochrome $b$ subunit of the formate dehydrogenase by formate and which acts as an electron mediator, MBP-NifL was significantly reduced in this in vitro assay. The kinetic of the respective reduction of NifL-bound FAD-cofactor was monitored by detecting the absorbance between 650 and $360 \mathrm{~nm}$ every $2 \mathrm{~min}$ as described in Experimental Procedures. As shown in Fig. 3.3, approximately 3.0 nmol MBP-NifL were reduced within 6 min after starting the reaction by the addition of $12.5 \mathrm{mM}$ formate to the assay. In the absence of formate no MBP-NifL reduction was observed. In the absence of DMN but in the presence of formate MBP-NifL reduction occurred about two orders of magnitude slower than with the presence of the red-ox mediator DMN (see Table 3.1, Exp. 3). To calculate the amount of FAD reduction of MBP-NifL by formate oxidation the absorbance decrease by time at $452 \mathrm{~nm}$ and the extinction coefficient for MBP-NifL bound FAD $\left(\Delta \varepsilon_{452 \mathrm{~nm}-600 \mathrm{~nm}}=6.63 \mathrm{mM}^{-1} \mathrm{x} \mathrm{cm}^{-1}\right)$ determined earlier was used. The UV-vis spectra in Fig. 3.3 further indicate that electrons derived from formate oxidation by formate dehydrogenase are subsequently transferred to DMN and later to the NifL-bound FAD, as no reduced cytochrome $b$ of formate dehydrogenase is visible (Fig. 3.3, absorbance at 424 and $552 \mathrm{~nm}$ ). 


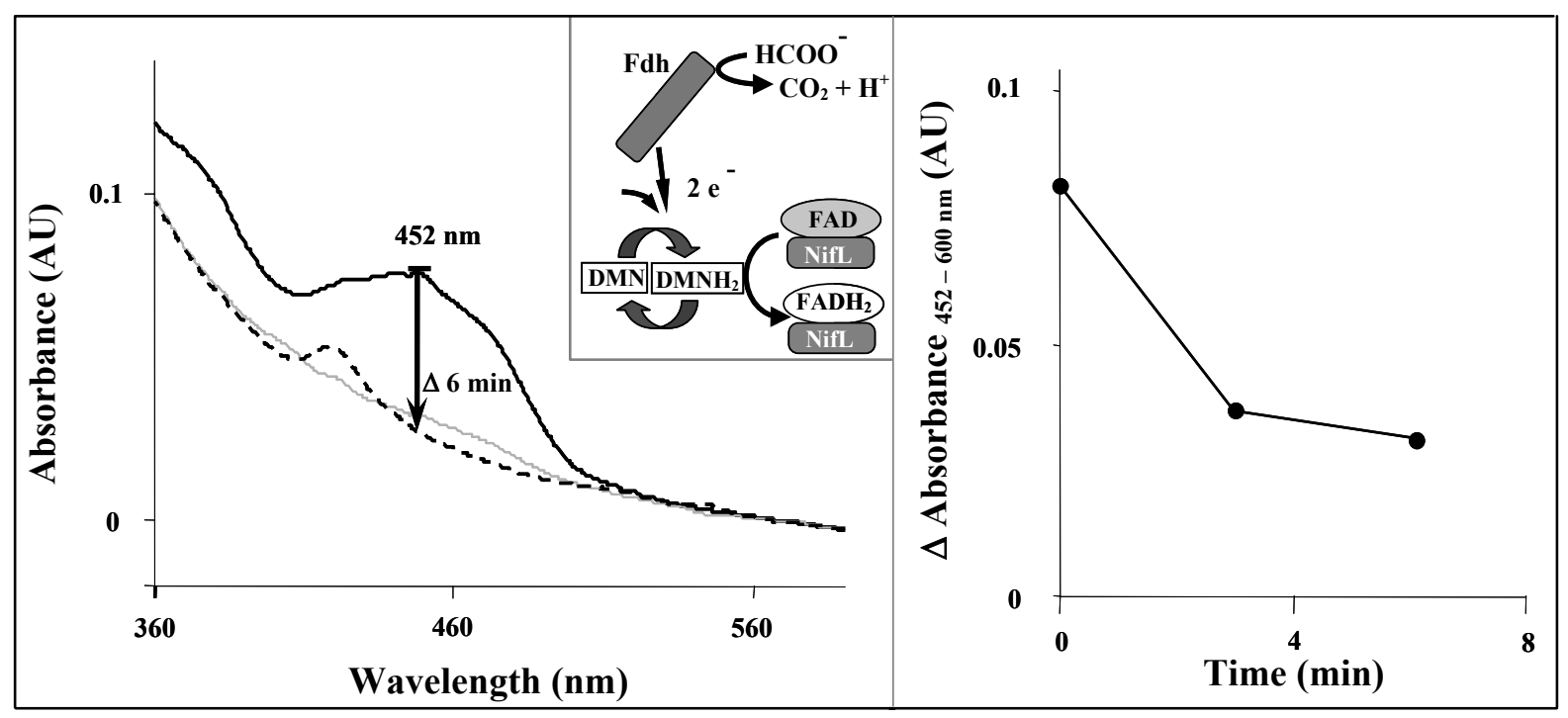

Figure 3.3: MBP-NifL reduction by formate in the presence of solubilised membranous formate dehydrogenase of $W$. succinogenes and DMN. Electrons derived from formate oxidation were transferred to the FAD-cofactor of purified MBP-NifL $(4.2 \mathrm{nmol})$ in the presence of solubilised Fdh isolated from $W$. succinogenes and in the presence of $0.2 \mathrm{mM}$ of the water soluble quinone analogon 2,3-dimethylnaphtoquinone (DMN) as electron mediator. UV-vis spectra were monitored every 2 or $3 \mathrm{~min}$ as described in Experimental Procedures. After 6 min reduction of approximately $3.0 \mathrm{nmol}$ MBP-NifL was achieved. In the left panel the original data of the initial spectrum (solid bold line), after $3 \mathrm{~min}$ (solid fine line) and after 6 min (dashed line) are indicated. The right panel shows the change in absorbance at $452 \mathrm{~nm}$ corrected for the absorbance at $600 \mathrm{~nm}$ in dependence to the incubation time. The electron flow in the in vitro system from formate to MBP-NifL is depicted in the cartoon.

MBP-NifL reduction driven by proteoliposomes containing partial reconstituted anaerobic respiratory chains of $W$. succinogenes. The next question we addressed was to analyze, whether MBP-NifL can be reduced by electrons derived from reconstituted anaerobic respiratory chains. In order to test whether an electron transfer from membrane-integrated reduced menaquinones to MBP-NifL can be achieved, proteoliposomes were prepared by inserting purified formate dehydrogenase or hydrogenase and menaquinones $\left(\mathrm{MK}_{6}\right.$ or $\mathrm{MMK}_{6}$ ), isolated from membranes of $W$. succinogenes into liposomes as described in Experimental Procedures. The components of the respiratory chain and their relative amounts were varied in order to further characterize the potential reduction and the rate of MBP-NifL reduction by electrons derived from the reconstituted anaerobic respiratory chains. The reduction of $4.2 \mathrm{nmol}$ MBP-NifL generally used in the $400 \mu \mathrm{l}$ test assays was studied under the respective conditions by monitoring the UV-vis-spectra every 2 min after starting the reaction by the addition of $12.5 \mathrm{mM}$ formate (final concentration). 
Table3.1: Characterization of in vitro reduction of $4.2 \mathrm{nmol}$ MBP-NifL (400 $\mu 1$ test volume) by formate oxidation using formate dehydrogenase (Fdh) from $W$. succinogenes

\begin{tabular}{|c|c|c|c|c|c|c|c|}
\hline $\begin{array}{l}\text { Experi- } \\
\text { ment }\end{array}$ & $\begin{array}{l}\text { Solubilised Fdh } \\
\text { amount }\end{array}$ & DMN amount & \multicolumn{2}{|c|}{$\begin{array}{c}\text { Initial FAD reduction } \\
\qquad \Delta \mathrm{E}_{\text {initial }}\left(\mathrm{min}^{-1}\right)^{1}\end{array}$} & $\underset{\text { (nmol) }}{\text { Reduced FAD }}$ & $\underset{(\min )}{\Delta \text { Time }}$ & $\begin{array}{l}\text { Depicted } \\
\text { in Figure }\end{array}$ \\
\hline 3 & $34 \mu \mathrm{g}$ & none & \multicolumn{2}{|c|}{0.00076} & 2.7 & $>45$ & $\begin{array}{c}\text { Not } \\
\text { shown }\end{array}$ \\
\hline 2 & $34 \mu \mathrm{g}$ & $0.2 \mathrm{mM}$ & \multicolumn{2}{|l|}{0.015} & 2.92 & 6.0 & Fig. 3.3 \\
\hline & $\begin{array}{c}\text { Fdh amount in } \\
\text { proteoliposomes } \\
\left.\text { (mg phospholipid }^{-1}\right)\end{array}$ & $\begin{array}{c}\text { MK }_{6} \text { amount } \\
\left.\text { (mg phospholipid }^{-1}\right)\end{array}$ & \multicolumn{2}{|c|}{$\begin{array}{c}\text { Initial FAD reduction } \\
\Delta \mathrm{E}_{\text {intial }}\left(\min ^{-1}\right)^{1}\end{array}$} & $\begin{array}{c}\text { Reduced FAD } \\
\text { (nmol) }\end{array}$ & $\underset{(\min )}{\Delta \text { Time }}$ & $\begin{array}{l}\text { Depicted } \\
\text { in Figure }\end{array}$ \\
\hline 5 & $13.6 \mu \mathrm{g}$ & $0.01 \mu \mathrm{mol}$ & 0.0036 & $1 \mathrm{x}^{2}$ & 2.8 & 21.0 & $\begin{array}{c}\text { Not } \\
\text { shown }\end{array}$ \\
\hline 6 & $27.2 \mu \mathrm{g}$ & $0.01 \mu \mathrm{mol}$ & 0.014 & $4 x^{2}$ & 3.11 & 8.0 & Fig. 3.5A \\
\hline 11 & $27.2 \mu \mathrm{g}$ & $0.026 \mu \mathrm{mol}$ & 0.032 & $9 x^{2}$ & 3.32 & 4.3 & Fig. $3.5 \mathrm{~B}$ \\
\hline 12 & $13.6 \mu \mathrm{g}$ & $0.026 \mu \mathrm{mol}$ & 0.0124 & $4 x^{2}$ & 2.84 & 12.0 & $\begin{array}{l}\text { Not } \\
\text { shown }\end{array}$ \\
\hline 9 & $27.2 \mu \mathrm{g}$ & none & 0.002 & $0.14 \mathrm{x}^{2}$ & 3.44 & 21.0 & Fig. 3.4 \\
\hline 10 & $13.6 \mu \mathrm{g}$ & none & 0.001 & $0.07 \mathrm{x}^{2}$ & 2.16 & 77.0 & $\begin{array}{l}\text { Not } \\
\text { shown }\end{array}$ \\
\hline 13 & $27.2 \mu \mathrm{g}$ & $0.017 \mu \mathrm{mol} \mathrm{MMK}_{6}$ & 0.015 & $4 x^{2}$ & 1.76 & 8.0 & $\begin{array}{c}\text { Not } \\
\text { shown }\end{array}$ \\
\hline
\end{tabular}

1 the reduction rates were calculated by determining the difference of the flavin-specific absorbance $2 \mathrm{~min}$ after formate addition.

${ }^{2}$ The reduction rates under the different conditions are indicated in relation to the reduction rate obtained under conditions present in exp. 5, which was set to 1.0.

Unexpectedly, in control assays using proteoliposomes, which solely contained purified formate dehydrogenase $(27.2 \mu \mathrm{g} / \mathrm{mg}$ phospholipids) in the absence of any additional menaquinones as electron carriers, significant reduction of MBP-NifL (3.44 nmol) was detected within a long time of $21 \mathrm{~min}$ as demonstrated in Fig. 3.4. This finding strongly indicates that electrons derived from formate oxidation have been finally transferred onto MBP-NifL. As seen from the spectrum (Fig. 3.4, absorbance at 424 and $552 \mathrm{~nm}$ ), cytochrome $b$ of the formate dehydrogenase was reduced within less than one min. It 
appears that the contaminating menaquinones, which are present in very small amounts in the fraction of purified formate dehydrogenase $(0.1-0.2 \mathrm{~mol} \mathrm{MK} \times \mathrm{mol}$ formate dehydrogenase $\mathrm{e}^{-1},{ }^{2}$ ), allowed the electron transfer from reduced cytochrome $b$ of formate dehydrogenase onto NifL by slow rates (insert left panel of Fig. 3.4, dashed line). This finding is further supported by the fact that isolated Triton-X100 solubilised formate dehydrogenase was also able to reduce MBP-NifL in the absence of the additional water soluble electron mediator DMN, however a reaction time longer than 45 min was required to reduce $2.7 \mathrm{nmol} \mathrm{MBP}-\mathrm{NifL}$ in the presence of formate and formate dehydrogenase (Table 3.1, experiment 3).

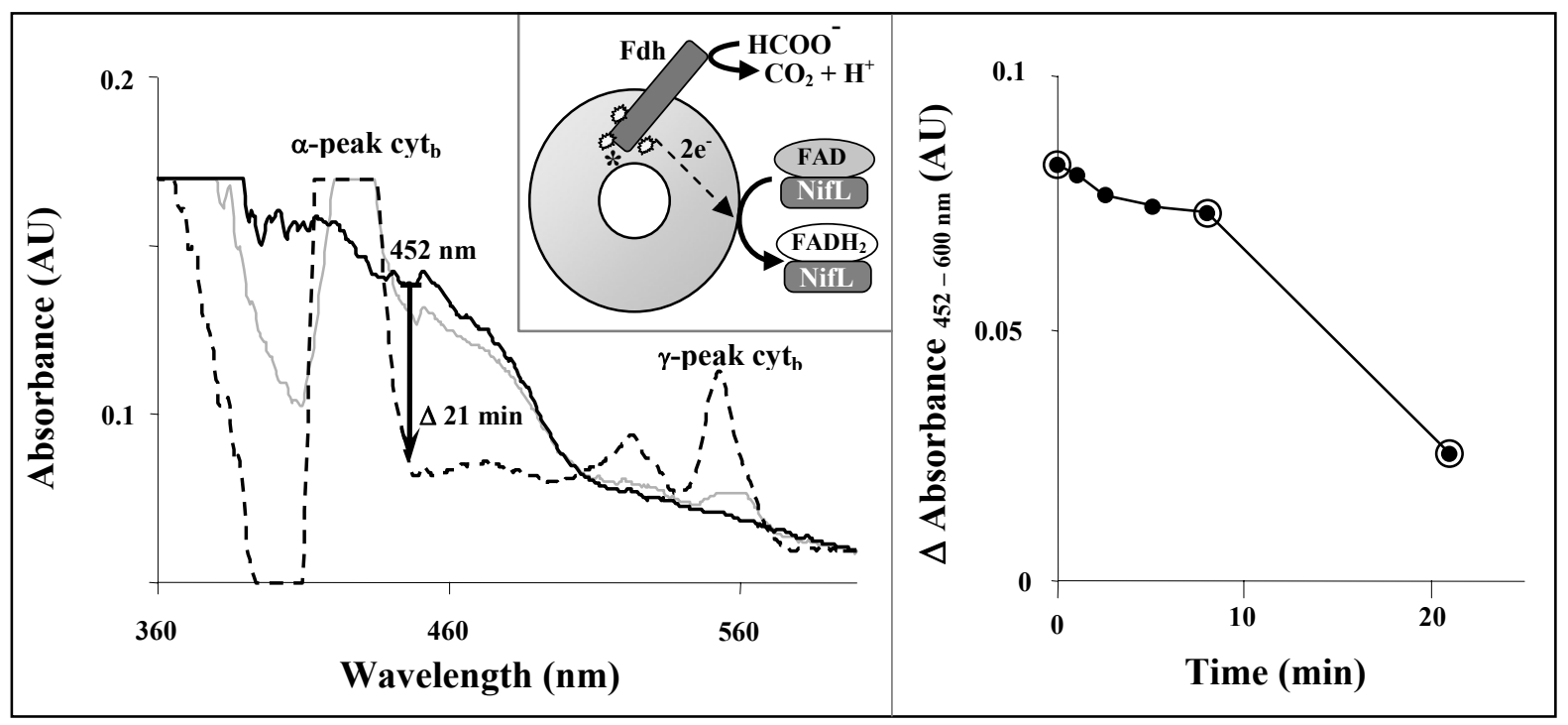

Figure 3.4: Reduction of MBP-NifL by formate oxidation using proteoliposomes containing purified formate dehydrogenase from $W$. succinogenes in the absence of any additional electron carrier. The FAD-cofactor of purified MBP-NifL (4.2 nmol) was reduced by electrons derived from formate oxidation by purified $W$. succinogenes formate dehydrogenase (Fdh) reconstituted into sonic liposomes ( $27.2 \mu \mathrm{g}$ formate dehydrogenase / $\mathrm{mg}$ phospholipid). The UVvis spectra were monitored every 3 to $10 \mathrm{~min}$. After $21 \mathrm{~min} 3.44 \mathrm{nmol}$ of MBP-NifL was reduced by electrons derived from formate oxidation. This is due to the presence of contaminating menaquinones $\left(^{*}\right)$ present in the purified formate dehydrogenase protein fraction $(0.1-0.2 \mathrm{~mol}$ MK $x$ mol formate dehydrogenase ${ }^{-1}$ ), which apparently allowed an electron transfer to NifL as indicated in the cartoon (dashed line). In the left panel the original data of the initial spectrum (bold dark line), after $8 \mathrm{~min}$ (fine grey line) and after $21 \mathrm{~min}$ (dashed bold line) are shown. The absorbance at $424 \mathrm{~nm}$ ( $\alpha$-peak) and $562 \mathrm{~nm}(\gamma$ peak) is due to the reduction of formate dehydrogenase cytochrome $b$, which occurs during the reduction process, indicating that the small amount of the menaquinone in the liposomal membrane is fully reduced. The right panel shows the change in absorbance at $452 \mathrm{~nm}$ corrected for the absorbance at $600 \mathrm{~nm}$ in dependence to the incubation time. The respective time points, for which the corresponding spectra are shown, are indicated by additional circles. The electron flow in the in vitro system from formate to MBP-NifL is depicted in the cartoon. 
When proteoliposomes were used in the in vitro assay, which contained in addition to formate dehydrogenase $\left(27 \mu \mathrm{g} / \mathrm{mg}\right.$ phospholipids) menaquinone $6\left(\mathrm{MK}_{6}\right)$ isolated from membranes of $W$. succinogenes $\left(0.01 \mu \mathrm{mol} \mathrm{MK}_{6} / \mathrm{mg}\right.$ phospholipid), MBP-NifL reduction was achieved significantly faster than in the absence of any added quinones (Table 3.1, experiment 6). As shown in Fig. 3.5A, 3.1 nmol MBP-NifL was reduced within 8 min after starting by the addition of formate. This finding indicates that the electrons from the reduced formate dehydrogenase cytochrome $b$ are transferred onto MBP-NifL in a much more efficient way via the additional menaquinones as electron carriers (insert left panel of Fig. 3.5A). The reduction rate was even further increased, when using higher amounts of menaquinones inserted into the lioposomes $\left(0.026 \mu \mathrm{mol} \mathrm{MK}_{6} / \mathrm{mg}\right.$ phospholipid) by a constant formate dehydrogenase content, reduction of $3.32 \mathrm{nmol}$ MBP-NifL was achieved within $4.3 \mathrm{~min}$ (see Fig. 3.5B). These findings strongly indicate that the reduction of MBPNifL is directly dependent on the menaquinone concentration in the membrane of the proteoliposomes. The reduction is further directly dependent on the formate dehydrogenase concentration in the proteoliposomes, as reducing the amount of formate dehydrogenase inserted in proteoliposomes to $13.6 \mu \mathrm{g} / \mathrm{mg}$ phospholipid resulted in a significant increase of reaction time for NifL reduction (Table 3.1, compare experiment 5 with experiment 6). Besides $\mathrm{MK}_{6}$, also 8-methyl-MK $\mathrm{MK}_{6}\left(\mathrm{MMK}_{6}\right)$, which comprises a much deeper redox potential, $(-220 \mathrm{mV})^{3}$, inserted into proteoliposomes together with formate dehydrogenase allowed the reduction of MBP-NifL in the in vitro assay with a comparable rate (see Table 3.1, compare experiments 13 and 6). The overall finding that electrons from reduced menaquinones of reconstituted partial anaerobic respiratory chains can be directly transferred onto MBP-NifL strongly supports our hypothesis that under oxygen limiting conditions NifL is able to receive electrons from the reduced menaquinone pool of the anaerobic respiratory chain in K. pneumoniae. 

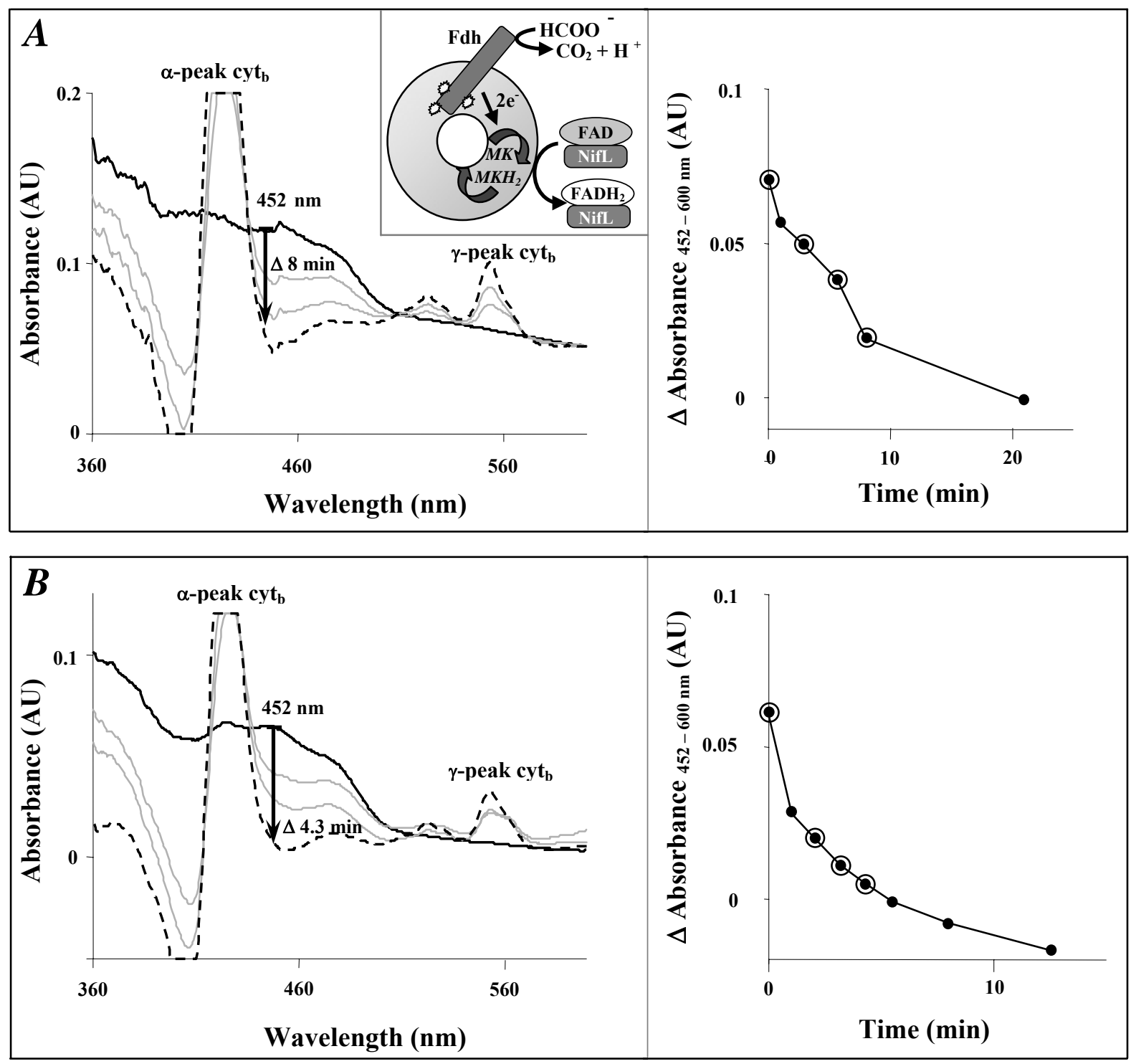

Figure 3.5: MBP-NifL reduction by formate oxidation using proteoliposomes containing purified formate dehydrogenase and $\mathbf{M K}_{\mathbf{6}}$ from $\boldsymbol{W}$. succinogenes. The NifL-bound FADcofactor $(4.2 \mathrm{nmol})$ was reduced by formate oxidation using proteoliposomes containing purified Fdh (27 $\mu \mathrm{g}$ formate dehydrogenase / mg phospholipid) and purified $\mathrm{MK}_{6}$ from $W$. succinogenes. MBP-NifL reduction was analyzed by monitoring the UV-vis spectra every $2 \mathrm{~min} .3 .11 \mathrm{nmol}$ MBP-NifL were reduced within $8 \mathrm{~min}$ when using $0.01 \mu \mathrm{mol} \mathrm{MK}_{6} / \mathrm{mg}$ phospholipid (A) and $3.32 \mathrm{nmol}$ within $4.3 \mathrm{~min}$ using $0.026 \mu \mathrm{mol} \mathrm{MK}_{6} / \mathrm{mg}$ phospholipid (B). In the left panels the original data are depicted: The initial spectra (bold dark lines); after $2.75 \mathrm{~min}$ and $5.7 \mathrm{~min}$ (A) and after $2 \mathrm{~min}$ and $3.15 \mathrm{~min}$ (B) (solid fine lines); and after $8 \mathrm{~min}$ (A) and after $4.3 \mathrm{~min}$ (B) (bold dashed line). The absorbance at $424 \mathrm{~nm}$ and $562 \mathrm{~nm}$ represents the reduction of Fdh cytochrome $b$. The right panels show the change in absorbance at $452 \mathrm{~nm}$ corrected for the absorbance at $600 \mathrm{~nm}$ in dependence to the incubation time; the respective time points, for which the corresponding spectra are shown, are indicated by additional circles. The electron flow in the in vitro system from formate to MBP-NifL is depicted in the cartoon. 
In order to compare the reduction rates for MBP-NifL in the in vitro assays using different amounts of formate dehydrogenase and $\mathrm{MK}_{6}$ inserted into the proteoliposomes, we determined the initial NifL reduction rates under the respective conditions. The initial reduction rates were calculated by determining the difference of the flavin-specific absorbance at $452 \mathrm{~nm}$ corrected for the absorbance at $600 \mathrm{~nm} 2$ min after formate addition. The rates obtained, which are summarized in Table 3.1 (initial FAD reduction), clearly demonstrate that the rate of NifL reduction is directly dependent on the formate dehydrogenase content and the menaquinone concentration in the proteoliposomal membrane. In general, an approximate 4-fold increase of the reduction rate was achieved by an twofold increase of the menaquinone concentration in the liposomal membrane (Table 3.1, compare experiments 5 and 12) or the formate dehydrogenase content in the proteoliposomes used (Table 3.1, compare experiments 5 and 6). Increasing both components in the membrane simultaneously resulted in a 9-fold increase of the reduction rate of MBP-NifL (Table 3.1, compare experiments 5 and 11). Comparing the reduction rates of MBP-NifL, when using protoeliposomes in the absence and presence of additional menaquinones, also clearly showed that the contaminating $\mathrm{MK}$ of purified formate dehydrogenase are not sufficient for high reduction rates and that an 7-fold increase in electron transfer rates is achieved in the presence of additional $\mathrm{MK}_{6}$ (Table 3.1, compare experiments 6 and 9).

As an independent control to confirm the data obtained, purified $W$. succinogenes hydrogenase and $\mathrm{MK}_{6}$ were inserted into liposomes. Using those proteoliposomes, MBPNifL reduction was achieved by hydrogen oxidation with comparable reduction rates as the ones determined with formate dehydrogenase (data not shown).

Association of reduced MBP-NifL to proteoliposomal membranes. Using two independent approaches, we have recently shown in vivo (i) that under derepressing conditions NifL is membrane-associated, allowing cytoplasmic NifA to activate nif-gene expression and (ii) that the membrane sequestration is directly dependent on FAD reduction (Grabbe and Schmitz, 2003; Klopprogge et al., 2002; Stips et al., 2004). In order to confirm our previous results we further analysed, whether MBP-NifL, which was reduced in the in vitro assay by electrons derived from formate oxidation by a reconstituted partial anaerobic respiratory chain is associated with the proteoliposomes or not. In order to study potential association of reduced MBP-NifL to proteoliposomal membranes we reduced $6.1 \mathrm{nmol}$ MBP-NifL with $15 \mathrm{mM}$ formate using proteoliposomes (6.25 $\mathrm{mg}$ phospholipids) 
containing formate dehydrogenase and $\mathrm{MK}_{6}(64 \mu \mathrm{g}$ formate dehydrogenase and $18 \mathrm{nmol}$ $\mathrm{MK}_{6}$ per mg phospholipid). After $20 \mathrm{~min}$ of anaerobic incubation the complete assay was applied to a $28 \mathrm{ml}$ sucrose gradient (10 - $40 \%$ sucrose in B-buffer) containing $15 \mathrm{mM}$ formate to keep MBP-NifL constantly in a reduced state. After ultracentrifugation for $4.5 \mathrm{~h}$ performed under anaerobic conditions, the different fractions $(1.5 \mathrm{ml})$ were analysed for (i) the presence of MBP-NifL by Western blot analysis using polyclonal antibodies raised against NifL and (ii) the presence of formate dehydrogenase activity as described in Experimental Procedures in order to detect fractions containing proteoliposomes with formate dehydrogenase. The results, which are depicted in Fig. 3.6, clearly demonstrated the simultaneous presence of reduced MBP-NifL and formate dehydrogenase in proteoliposomes mainly in fractions 4 - 8 (Fig. 3.6 D). However, when oxidized MBP-NifL and proteoliposomes were analyzed in the presence of oxygen the majority of MBP-NifL was found in fractions 9 - 16 (Fig. 3.6 C) as was the case for oxidized MBP-NifL in the absence of proteoliposomes (Fig. 3.6 B). The finding that exclusively reduced MBP-NifL is associated to the proteoliposomes strongly confirms our previous in vivo results that NifL is membrane associated under derepressing conditions. It further clearly demonstrates that no additional K. pneumoniae specific protein is required for NifL reduction or membrane association of reduced NifL. 
A
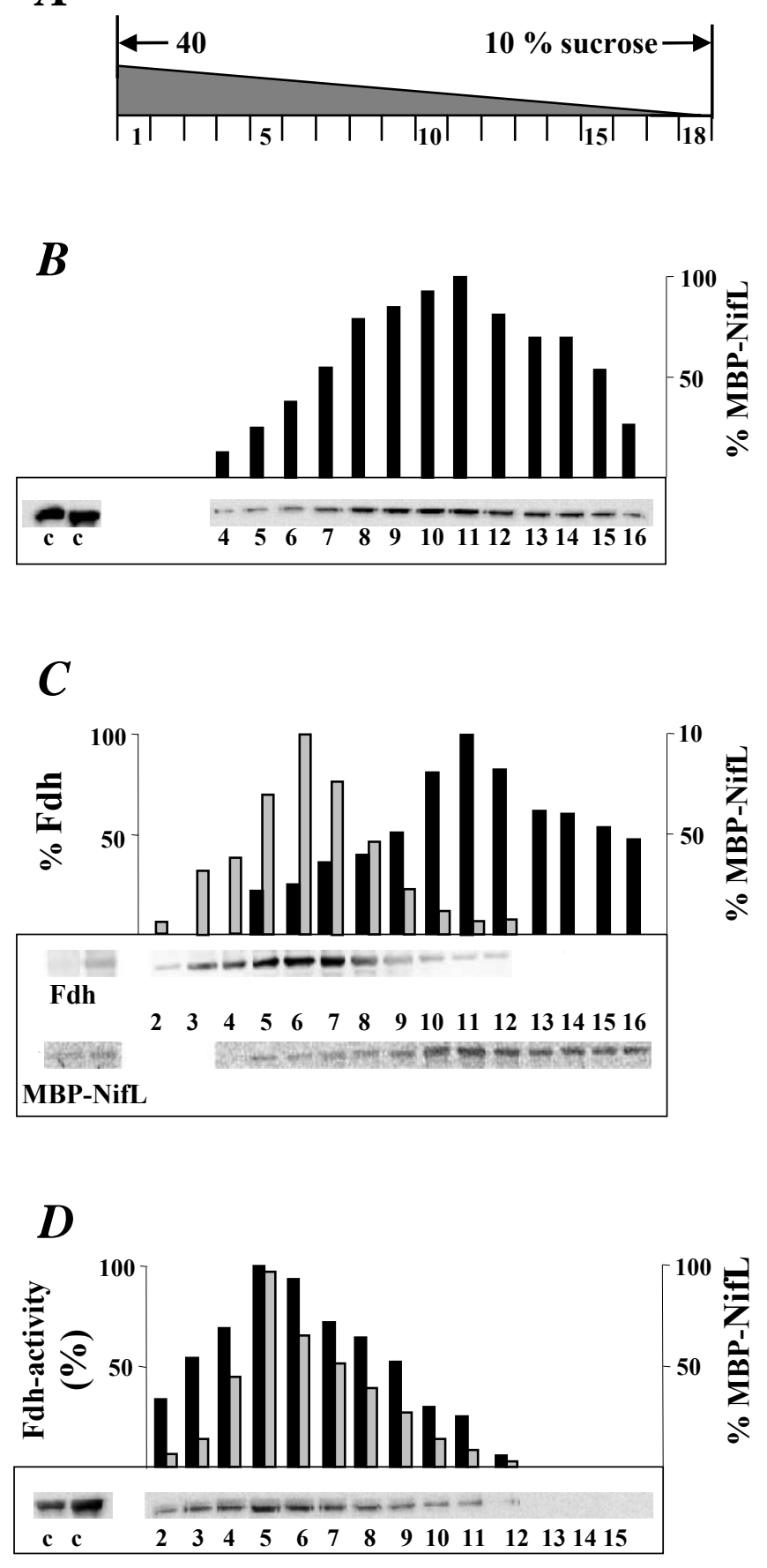

Figure 3.6: Analysis of association of in vitro reduced MBP-NifL to proteoliposomal membranes. MBP-NifL $(610 \mu \mathrm{g})$ was loaded onto a $28 \mathrm{ml}$ sucrose gradient (10 to $40 \%$ sucrose in B-buffer) followed by ultracentrifugation for $4.5 \mathrm{~h}$ at $175.000 \mathrm{x} \mathrm{g}$. The respective fractions of the gradient depicted in (A) were analysed for the presence of MBP-NifL by Western blot analysis using antibodies directed against NifL. In general, fractions of one experiment were analyzed on the same polyacrylamide gel together with purified MBP-NifL as control. (B) Ultracentrifugation of MBP-NifL under aerobic conditions in the absence of proteoliposomes. Original Western blot data (bottom) and relative amounts of NifL in the respective fractions setting the NifL amount of 
fraction 11 to $100 \%$ (black bars); (c), 210 and 260 ng purified MBP-NifL. (C) Ultracentrifugation of MBP-NifL under aerobic conditions in the presence of proteoliposomes: Original Western blot data (bottom) and relative amounts of NifL and Fdh in the respective fractions, setting the NifL amount of fraction 11 to $100 \%$ (black bars) and the Fdh amount of fraction 6 to $100 \%$ (grey bars). (D) Anaerobic ultracentrifugation of MBP-NifL after in vitro reduction by proteoliposomes (see Experimental Procedures). The relative amounts of NifL setting the NifL amount of fraction 5 to $100 \%$ are indicated as black bars above the original Western blot data. The Fdh activity of the respective fractions was determined as described in Experimental Procedures. The relative amounts are indicated as grey bars setting the Fdh activity of fraction $5(97 \mathrm{U} / \mathrm{ml})$ to $100 \%$.

\section{Discussion}

The enzymatic reduction of molecular nitrogen to ammonia is an extremely energy consuming process, and the presence of oxygen causes the catalyzing nitrogenase complex to be rapidly and irreversible inactivated (Burgess and Lowe, 1996; Rees and Howard, 1999). As a consequence diazotrophs tightly control both, the synthesis and activity of nitrogenase in response to environmental changes in oxygen and ammonium availability. In K. pneumoniae, nif-genes are exclusively expressed under oxygen and concurrent nitrogen limitation (reviewed in (Martinez-Argudo et al., 2004c; Schmitz et al., 2002)). Here the key regulatory mechanism for nif-gene induction by the transcriptional activator NifA is membrane sequestration of the reduced negative regulator NifL under simultaneous oxygen and nitrogen limitation (Grabbe and Schmitz, 2003; Klopprogge et al., 2002; Stips et al., 2004). The focus of this work was to investigate the proposed electron transfer from the anaerobic respiratory chain onto NifL resulting in membrane association of NifL.

Demonstration of a direct electron transfer from reduced menaquinones of artificial membrane systems to NifL. Using reconstituted partial anaerobic respiratory chains of $W$. succinogenes as artificial membrane systems consisting of defined components we revealed strong evidence that electrons are directly transferred from reduced menaquinone pools in membranes to the FAD-cofactor of soluble NifL (Figs. 3.4, 3.5). This is consistent with previous experiments, in which NifL reduction has been achieved by the anaerobic respiratory chain of inside-out vesicles derived from anaerobic K. pneumoniae cells after the addition of $\mathrm{NADH} / \mathrm{H}^{+}$(Grabbe and Schmitz, 2003). The current experimental data 
clearly demonstrate for the first time that NifL is able to receive electrons directly from reduced menaquinones in membranes in the absence of any additional oxidoreductase or any $K$. pneumoniae specific protein. This strongly indicates that neither a NifL-specific membrane bound receptor is required for NifL contacting the cytoplasmic membrane nor is a K. pneumoniae specific electron carrier or oxidoreductase required for the reduction of the NifL-bound FAD-cofactor. This is further supported by the results obtained from a saturated transposon mutagenesis, in which despite components of the anaerobic respiratory chains of K. pneumoniae, FNR and essential genes for nitrogen fixation (e.g. nif genes, $n \operatorname{tr} B, n \operatorname{tr} C, g \ln K)$ no further specific proteins were identified to be essential for NifL reduction $^{4}$.

Sucrose gradient ultracentrifugation analysis further showed that the majority of NifL reduced by the artificial membrane systems stays associated to the proteoliposomes (Fig. 3.6 D). In the presence of oxygen, however, oxidized NifL was mainly present in fractions with significantly lower sucrose concentration not containing the proteoliposomes (Fig. 3.6 C). This finding that only reduced NifL stays associated to the proteoliposomes in vitro is in accordance with our previous in vivo results on NifL localisation in response to oxygen availability (Klopprogge et al., 2002) (see Fig. 3.1). NifL presumably contacts the cytoplasmic membrane with its hydrophobic N-terminal domain based on either ionic or hydrophobic interactions. However, at the current experimental status we are not able to analyze (e.g. by incorporation of protonophores), whether membranes have to be energized or not for NifL contact. This is due to the fact that the oxidoreductases (formate dehydrogenase and hydogenase) were inserted into the proteoliposomes by the method of freeze and thawing leading to a more or less random orientation of the enzymes in the proteoliposomes and to more or less non proton dense liposomes, that possibly do not generated a membrane potential. For a distinct orientation of the dehydrogenases in the liposomal membranes and for a good impermeability of protons experimentally difficult methods with a high investment of time and work are necessary, e.g. preparation methods of proteoliposomes according to Lambert et al. (Lambert et al., 1998).

Following changes from aerobic to anaerobic environmental conditions under nitrogen limitation, rapid reduction of NifL is important to sequester the negative regulator to the membrane. The rates of NifL reduction achieved in the in vitro system were calculated to be approx. 2 nmol NifL per min when highest amounts of quinones were used in the proteoliposomes with a $\mathrm{MK}_{6}$ to NifL ratio in the range of 30 to 1 (experiment 11). Under nitrogen limitation approximately 100 molecules of NifL are present in a single 
K. pneumoniae cell ${ }^{5}$, whereas the amount of reduced menaquinones in the membranes in the steady state of anaerobic respiration is approximately three orders of magnitude higher. This in vivo ratio should allow much higher rates of NifL reduction in vivo than obtained in vitro; consequently, under derepressing conditions each NifL protein of a cell, which is contacting the cytoplasmic membrane, will immediately receive electrons from the reduced menaquinone pool. Thus, this unspecific electron transfer from reduced menaquinones onto NifL under physiological conditions should be sufficient to sequester NifL to the membrane and lead to a nif-gene induction in a short time.

A comparable regulatory mechanism has been described for the proline utilization protein A (PutA) in Escherichia coli and Salmonella typhimurium. The flavoprotein PutA acts as a transcriptional repressor of the proline utilization (put) operon by binding to an operator, when intracellular proline concentrations are low (Ostrovsky de Spicer et al., 1991b; Ostrovsky de Spicer and Maloy, 1993; Wood, 1987). However, high intracellular proline concentration have been shown to alter the function of PutA driven by the reduction of PutA-bound FAD (Brown and Wood, 1993; Zhang et al., 2004; Zhang et al., 2007). Reduced PutA associates with the membrane and catalyzes the two-step oxidation of proline to glutamate. Membrane sequestration of PutA disrupts the PutA-DNA complex and consequently induces the put-gene expression (Maloy, 1987; Muro-Pastor et al., 1997; Zhang et al., 2004).

In contrast to K. pneumoniae NifL, membrane-association has not been demonstrated for the redox-sensitive NifL protein of $A$. vinelandii, nor has the physiological electron donor been identified yet. It is currently proposed that under anaerobic conditions reduction of $A$. vinelandii-NifL occurs non-specifically and dependent on the availability of reducing equivalents in the cytoplasm (Dixon, 1998; Little et al., 2006; Macheroux et al., 1998; Martinez-Argudo et al., 2005). Under simultaneous nitrogen limitation, the NifL conformation with a reduced FAD moiety does not allow to interact and inhibit cytoplasmic NifA. However, upon oxidation of its FAD moiety NifL undergoes a conformational change and is competent to inhibit NifA irrespectively of the nitrogen status (reviewed in (Little et al., 2006; Martinez-Argudo et al., 2005)).

Hypothetical model: Menaquinones as the red-ox signal for $K$. pneumoniae NifL. In previous work we hypothesized that the nif-gene regulation is based on NifL monitoring the reduction state of components of the anaerobic respiratory chain. The presented work now clearly demonstrates a direct electron transfer from reduced menaquinones onto NifL 
and provides evidence for a direct link of the red-ox status of the menaquinone pool to nif gene regulation. Thus, the reduced menaquinone pool appears to act as the signal for anaerobiosis. To our knowledge only very few examples are known, for which the red-ox state of the quinone pool is proposed as red-ox signal for downstream signal transduction to regulate gene expression: (i) The Arc two-component regulatory system of E. coli (Georgellis et al., 2001), (ii) histidine kinases BvgS and EvgS of Bordetella pertussis and E. coli (Bock and Gross, 2002) and (iii) RegB of Rhodobacter capsulatus (Swem et al., 2006). Georgellis and collegues showed that in E. coli oxidized quinones act as direct negative signal inhibiting autophosphorylation of ArcB by oxidizing two red-ox active cysteine residues leading to an intermolecular disulfide bond formation (Malpica et al., 2004). In case of the global red-ox two component regulatory system RegA/RegB of $R$. capsulatus Bauer and co-workers very recently demonstrated that autophosphorylation of the sensor kinase RegB in vitro is inhibited by the addition of oxidized coenzyme $\mathrm{Q}_{1}$, whereas reduced coenzyme $\mathrm{Q}_{1}$ exhibits no inhibitory effect on kinase activity (Swem et al., 2006). In contrast to those red-ox signalling processes, in which quinones provide the oxidative power under aerobic conditions, we now revealed strong evidence that in K. pneumoniae the reducing power of menaquinones is involved in red-ox signalling for nif-gene regulation. We further propose that the electron transfer from the menaquinone pool to NifL occurs by direct contact of reduced menaquinone and the regulatory protein. However, specific menaquinone binding sites, which are known for transmembrane domains of components of respiratory complexes (Gross et al., 2004; Iverson et al., 1999; Matsson et al., 2000), have not been identified yet in NifL. At the current experimental status we cannot completely exclude that NifL reduction also occurs by electrons derived from the ubiquinone pool, which might be in equilibrium with the menaquinone pool by a MQ:UQ oxidoreductase in K. pneumoniae. However, under aerobic conditions, this will not allow to sequester NifL to the cytoplasmic membrane, as reduced NifL will be rapidly re-oxidized by molecular oxygen. Linking the regulatory mechanism of NifL to the anaerobic respiratory chain and the reducing power of the respective menaquinone pool (see Fig. 3.1), further allows the possibility to integrate the energy status of the cell in addition to the signal of oxygen status. This is particularly attractive as nitrogen fixation is a high energy and high reducing power consuming process (Rees and Howard, 1999). 


\section{Footnotes}

This work was supported by the Deutsche Forschungsgemeinschaft (SCHM1052/8-1) and the Fonds der Chemischen Industrie.

${ }^{1}$ The abbreviations used are: DMN, dimethylnaphtoquinone; FAD, flavin adenine dinucleotide; $\mathrm{MMK}_{6}, 8$-methyl-menaquinone $6{ }_{6} \mathrm{MK}_{6}$, menaquinone ${ }_{6}$

${ }^{2}$ O. Klimmek, unpublished results

${ }^{3}$ O. Klimmek \& Mac Millan, unpublished results

${ }^{4}$ J. Stips \& R.A. Schmitz, upublished results

${ }^{5}$ R. Thummer \& R.A. Schmitz, upublished data 
Chapter 4:

\section{Analysis of membrane association of $K$. pneumoniae NifL: A mutational analysis of the regulatory protein}

\section{Summary}

In Klebsiella pneumoniae nitrogen fixation is tightly controlled in response to the availability of ammonium and the presence of molecular oxygen. Under repressing conditions, NifL directly inhibits the nif specific transcriptional activator NifA by direct protein-protein interaction and thus nif-gene transcription is repressed. Under nitrogen fixing conditions, the reduced flavoprotein NifL is sequestered to the cytoplasmic membrane, impairing the inhibition of cytoplasmic NifA. We report here on a random mutagenesis of NifL to identify amino acids essential for membrane association or binding the FAD-cofactor. Overall, 11.500 clones of three independently generated pools of mutant NifL were screened for mutants conferring a nif--phenotype under nitrogen fixing conditions. 26 mutants showing no nitrogen fixation at all and 41 mutants showing a significantly reduced nitrogen fixation were identified and confirmed in several independent screening steps. By sequence analysis amino acid residues located mainly within the N-terminal domain were identified in the various mutants and selected for separation by site directed mutagenesis.

\section{Introduction}

In the free living nitrogen fixing K. pneumoniae the highly energy consuming process of nitrogen fixation is strictly regulated by the environmental signals molecular oxygen and ammonium availability to avoid unnecessary consumption of energy (Burgess and Lowe, 1996; Rees and Howard, 1999; Schmitz et al., 2002). Only in the absence of combined nitrogen and simultaneous absence of oxygen, $K$. pneumoniae is able to fix molecular nitrogen (Hill et al., 1981; Merrick, 1982; Merrick and Edwards, 1995; Schmitz et al., 
2002). Regulation of the nif-specific transcriptional regulator NifA in response to oxygen and nitrogen availability mediated by NifL is achieved by direct protein-protein interaction under conditions inappropriate for nitrogen fixation (Govantes et al., 1998; Henderson et al., 1989; Stips et al., 2004). Under nitrogen fixing conditions NifA induces nif-gene expression by activating the alternative $\sigma^{54}$ RNA-Polymerase (Hoover et al., 1990; Morett and Buck, 1989). We have recently demonstrated that upon anaerobiosis and simultaneous absence of combined nitrogen the flavoprotein NifL is sequestered to the cytoplasmic membrane releasing NifA inhibition in the cytoplasm (Grabbe and Schmitz, 2003; Klopprogge et al., 2002). We further obtained that membrane sequestration of NifL is achieved by the reduction of the N-terminally bound FAD-cofactor by electrons derived from the reduced menaquinone pool (Grabbe and Schmitz, 2003; Thummer et al., 2007). In vitro assays with reconstituted anaerobic respiratory chains of Wolinella succinogenes demonstrated that the electron transfer onto NifL occurs unspecifically as no further oxidoreductase or K. pneumoniae specific receptor protein present in the cytoplasmic membrane is required for FAD reduction (Thummer et al., 2007). This membrane association of the negative regulator NifL, leading to active NifA in the cytoplasm, is the key regulatory mechanism for regulating nitrogen fixation in K. pneumoniae in response to the environmental signals oxygen and nitrogen availability (Klopprogge et al., 2002; Stips et al., 2004).

For Azotobacter vinelandii, another free living organism capable of nitrogen fixation regulating nif-gene transcription by a nifLA operon, NifA activity is also regulated by direct protein-protein interaction with the negative regulator NifL (Lei et al., 1999; Money et al., 1999; Money et al., 2001). In contrast to K. pneumoniae it has not been shown for A. vinelandii, that a change in the localization of NifL is responsible for the activation of nif-gene expression. Although A. vinelandii NifL exhibits approximately $30 \%$ homology and $50 \%$ similarity to K. pneumoniae NifL on amino acid level the negative regulator is located in the cytoplasm under all conditions. The electrons for FAD reduction are presumably derived from reduction equivalents present in the cytoplasm during anaerobiosis (Dixon, 1998; Macheroux et al., 1998).

The nitrogen signal is transduced towards the NifL/NifA system by the PII-like nitrogen regulatory protein GlnK which senses the cellular nitrogen status (Arcondeguy et al., 2000; Arcondeguy et al., 2001; Atkinson and Ninfa, 1999; He et al., 1998; Jack et al., 1999; van Heeswijk et al., 1996; Xu et al., 1998). In K. pneumoniae it was demonstrated that GlnK is required for the relief of NifL inhibition under nitrogen limiting conditions (Arcondeguy et 
al., 1999; Arcondeguy et al., 2000; He et al., 1998; Jack et al., 1999). Recently pull-down experiments revealed that under nitrogen limiting conditions GlnK interacts with both regulatory proteins, NifL and NifA, forming transient trimeric complexes, and thus effecting the formation of the NifL/NifA complex. As a result the inhibitory NifL-NifA complex dissociates and NifL is sequestered to the cytoplasmic membrane if molecular oxygen is not available (Klopprogge et al., 2002; Stips et al., 2004).

In order to get deeper insights into NifL structure and function the nifL gene was randomly mutagenized by PCR amplification under reduced Taq-polymersase fidelity (Leung et al., 1989) to identify amino acid residues involved in either membrane association or in binding the FAD-cofactor. A screening system was established to identify nifL mutants which are no longer sequestered to the cytoplasmic membrane under derepressing conditions thus constitutively inhibiting NifA-activity resulting in a nif these conditions exclusively mutations in the N-terminal domain of NifL should result in a nif ${ }^{-}$-phenotype when due to the amino acid exchanges (i) the coordination of the FADcofactor is affected, (ii) mutated amino acids on the protein surface effect contacting the cytoplasmic membrane (iii) the electron transfer onto NifL is negatively influenced. Those mutations were separated by site-directed mutagenesis and further analyzed.

\section{Materials and Methods}

Bacterial Strains and Plasmids. The bacterial strains and plasmids used in this work are listed in Table 1. Plasmid DNA was transformed into E. coli and into K. pneumoniae cells according to the method of Inoue et al. (Inoue et al., 1990).

Construction of pRS315: A $1.7 \mathrm{kbp}$ PCR fragment carrying nifL under the control of the T7 promoter was generated using the Taq-polymerase for amplification, pJES283 as template and a set of primers, of which the sense primer was overlaying the T7-promoter region of the P(T7)-nifL construct in pJES283 (5'TAATATCGACTCACTATAGGGAGACC3', sense primer; 5'TAAACTGCTGGGAGAGATCGAAAC3', antisense primer). The resulting $1.7 \mathrm{kbp}$ PCR product was cloned into the TOPO TA-cloning vector pCRII-TOPO (Invitrogen) and the plasmid was designated pRS315 (Fig. 4.1). 
Table 4.1: Bacterial strains and plasmids

\begin{tabular}{|c|c|c|}
\hline Strain / plasmid & Relevant genotype or description & Source, reference \\
\hline \multicolumn{3}{|l|}{ Strains: } \\
\hline \multicolumn{3}{|l|}{ K. pneumoniae } \\
\hline M5a1 & Wild type & \\
\hline UN4495 & $\begin{array}{l}\phi(\text { nifK-lacZ)5935 } \Delta l a c-4001 \text { his } \\
\text { D4226 Gal }\end{array}$ & $\begin{array}{l}\text { (MacNeil et al., } \\
\text { 1981) }\end{array}$ \\
\hline K2674 & M5a1 + pRS315 & This study \\
\hline clone I (= K2844) & M5a1 + pRS315* (NifL Q57L) & This study \\
\hline clone II (= K2841) & M5a1 + pRS315* (NifL R80C) & This study \\
\hline clone XXI (= K 2885) & M5a1 + pRS315* (NifL R335G) & This study \\
\hline clone XXII (= K 2887) & $\mathrm{M} 5 \mathrm{a} 1+\mathrm{pRS} 315^{*}(\mathrm{NifL}$ T478A) & This study \\
\hline clone XXIII (= K 2901) & $\mathrm{M} 5 \mathrm{a} 1+\mathrm{pRS} 315^{*}(\mathrm{NifL}$ D353G) & This study \\
\hline clone XXIV & M5a1 + pRS315* (NifL E285G) & This study \\
\hline clone XXV & M5a1 + pRS315* (NifL Q273R) & This study \\
\hline \multicolumn{3}{|l|}{ E. coli } \\
\hline DH5 $\alpha$ & General cloning strain & $\begin{array}{l}\text { (Miller and } \\
\text { Mekalanos, 1988) }\end{array}$ \\
\hline \multicolumn{3}{|l|}{ Plasmids: } \\
\hline pCRII & TOPO-TA cloning vector & Invitrogen \\
\hline pJES283 & $\begin{array}{l}\mathrm{P}(\mathrm{T} 7)-n i f L / n i f A_{(100 \mathrm{bp})} \text { fragment in } \\
\text { pT7-7 }\end{array}$ & (Lee et al., 1993b) \\
\hline pRS315 & $\begin{array}{l}\mathrm{P}(T 7) \text {-nifL fragment (pJES283) in } \\
\text { pCRII }\end{array}$ & This study \\
\hline
\end{tabular}

Growth conditions. K. pneumoniae wild type and mutant strains were aerobically grown on Luria Bertani (LB) medium at $37{ }^{\circ} \mathrm{C}$. Under nitrogen limiting conditions cells were grown anaerobically on minimal medium supplemented with $0.5 \%$ sucrose as sole carbon and energy source at $30{ }^{\circ} \mathrm{C}$ in closed bottles with molecular nitrogen $\left(\mathrm{N}_{2}\right.$, grade 5.0, Linde, Germany) in the gas phase (at least 0.2 bar over normal pressure). The medium was further supplemented with $0.3 \mathrm{mM}$ sulfide and $0.002 \%$ resazurin to monitor anaerobiosis (Schmitz et al., 1996). Overnight precultures were grown anaerobically in the same 
medium containing $4 \mathrm{mM}$ glutamine as nitrogen source lacking sulfide and resazurin and were used to inoculate main cultures. The growth of the main cultures was then monitored at $600 \mathrm{~nm}$ for at least $60 \mathrm{~h}$.

For solid medium purified agar (Difco, Difco Laboratories, Detroit, USA) was used to avoid contamination with organic nitrogen and cells were incubated in anaerobic pots for 6 days at $30{ }^{\circ} \mathrm{C}$ with molecular nitrogen in the gas phase. To ascertain the availability of molecular nitrogen for nitrogen fixation 0.5 bar $\mathrm{N}_{2}\left(\mathrm{~N}_{2}\right.$, grade 5.0, Linde, Germany) was added once a day.

Generating mutant pools of $\boldsymbol{K}$. pneumoniae nifL. The DNA region of pJES283 carrying nifL under the control of the $T 7$ promoter (P(T7)nifL) (Fig. 4.1) was amplified at $53{ }^{\circ} \mathrm{C}$ using the primer set nifLT7for and nifLT7rev in the presence of $80 \mu \mathrm{M} \mathrm{MnCl}_{2}$, leading to reduced Taq-polymerase fidelity (Leung et al., 1989) (see Table 4.2). The PCR fragments were purified and TA-cloned into TOPO TA-cloning vector pCRII-TOPO (Invitrogen). The resulting plasmids, pRS315*, carrying the mutated nifL-gene were transferred into E. coli DH5 $\alpha$ and the clones were separated on solid medium selecting for kanamycin and ampicillin resistance conferred by the plasmid. Three independently generated pRS315* plasmid pools of mutated nifL were constructed derived from 3.494 (RT1), 4347 (RT2) and 4.400 (RT3) clones respectively. To determine the range of the mutation frequencies 20 individual plasmids were randomly chosen and the respective nifL gene was sequenced.

Table 4.2: List of primers used for PCR amplification, sequencing and site-directed mutagenesis

\begin{tabular}{ccl}
\hline Description & $\begin{array}{c}\text { Amino acid } \\
\text { mutation }\end{array}$ & \\
\hline niflT7 for & none & TAATACGACTCACTATAGGGAGACC \\
niflT7 rev & none & TAAACTGCTGGGAGAGATCGAAAC \\
M13 For(-20) & none & GTAAAACGACGGCCAG \\
TOPO & & \\
M13 Rev TOPO & none & CAGGAAACAGCTATGAC \\
nifL-int-for & none & CATATGACGCTAATGGAAGCG \\
nifL mut 1 for & V31A & GCTGTTTTTTACAATGGCCGAACAGGCATCG \\
nifL mut 1 rev & V31A & CGATGCCTGTTCGGCCATTGTAAAAAACAGC
\end{tabular}




\begin{tabular}{|c|c|c|}
\hline nifL mut 2 for & Q33R & CAATGGTCGAACGGGCATCGGTAGCG \\
\hline nifL mut 2 rev & Q33R & CGCTACCGATGCCCGTTCGACCATTG \\
\hline nifL mut 3 for & N46D & GATGCCCGGGCGGATATTATCTACGC \\
\hline nifL mut 3 rev & N46D & GCGTAGATAATATCCGCCCGGGCACT \\
\hline nifL mut 4 for & Q57L & GCGTTTTGCCGCCTGACTGGATACTCG \\
\hline nifL mut 4 rev & Q57L & CGAGTATCCAGTCAGGCGGCAAAACGC \\
\hline nifL mut 5 for & L62P & GGATACTCGCCGGCGCAATTGC \\
\hline nifL mut 5 rev & L62P & GCAATTGCGCCGGCGAGTATCC \\
\hline nifL mut 6 for & Q64R & CTCGCTGGCGCGATTGCTCAATC \\
\hline nifL mut 6 rev & Q64R & GATTGAGCAATCGCGCCAGCGAG \\
\hline nifL mut 7 for & $\mathrm{R} 80 \mathrm{C}$ & GCCAGACGCCGTGCGAGATCTACCAG \\
\hline nifL mut $7 \mathrm{rev}$ & $\mathrm{R} 80 \mathrm{C}$ & CTGGTAGATCTCGCACGGCGTCTGGC \\
\hline nifL mut 8 for & $\mathrm{I} 82 \mathrm{~T}$ & CGCCGCGCGAGACCTACCAGGAGATGTGG \\
\hline nifL mut 8 rev & $\mathrm{I} 82 \mathrm{~T}$ & CCACATCTCCTGGTAGGTCTCGCGCGGCG \\
\hline nifL mut 9 for & I116V & GGTAGATATCGATGTCACGCCGGTGCTG \\
\hline nifL mut 9 rev & I116V & CAGCACCGGCGTGACATCGATATCTACC \\
\hline nifL mut 10 for & A45V & CCGATGCCCGGGTGAATATTATCTACG \\
\hline nifL mut 10 rev & A45V & CGTAGATAATATTCACCCGGGCATCGG \\
\hline nifL mut 11 for & N67S & GCGCAATTGCTCAGTCAAAACCCGCGC \\
\hline nifL mut $11 \mathrm{rev}$ & N67S & GCGCGGGTTTTGACTGAGCAATTGCGC \\
\hline nifL mut 12 for & Q88R & CAGGAGATGTGGCGAACCCTGCTCCAGC \\
\hline nifL mut 12 rev & Q88R & GCTGGAGCAGGGTTCGCCACATCTCCTG \\
\hline nifL mut 13 for & G98S & GCCGTGGCGCAGTCAGCTAATTAATCAGC \\
\hline nifL mut 13 rev & G98S & GCTGATTAATTAGCTGACTGCGCCACGGC \\
\hline nifL mut 14 for & L193Q & CGGGAAAGAGCTGCAGGTCGAGCTCCAGG \\
\hline nifL mut 14 rev & L193Q & CCTGGAGCTCGACCTGCAGCTCTTTCCCG \\
\hline nifL mut 15 for & $\mathrm{K} 271 \mathrm{E}$ & GGCTCGACCGTCTGGAACAGCAAATGACC \\
\hline nifL mut 15 rev & K271E & GGTCATTTGCTGTTCCAGACGGTCGAGCC \\
\hline nifL mut 16 for & Q86L & CGCGAGATCTACCTGGAGATGTGGCAAAC \\
\hline nifL mut 16 rev & Q86L & GTTTGCCACATCTCCAGGTAGATCTCGCGC \\
\hline nifL mut 17 for & E191G & CTGCGGCGGGAAAGGGCTGCTGGTCGAGC \\
\hline nifL mut $17 \mathrm{rev}$ & E191G & GCTCGACCAGCAGCCCTTTCCCGCCGCAG \\
\hline nifL mut 18 for & E195G & GAGCTGCTGGTCGGGCTCCAGGTTTCCC \\
\hline nifL mut 18 rev & E195G & GGGAAACCTGGAGCCCGACCAGCAGCTC \\
\hline
\end{tabular}




$\begin{array}{lll}\text { nifL mut 21 for } & \text { R335G } & \text { CGCCTGCAGCGCTGCGGCCCTTCTCTTGAGC } \\ \text { nifL mut 21 rev } & \text { R335G } & \text { GCTCAAGAGAAGGGCCGCAGCGCTGCAGGC } \\ & & \text { G } \\ \text { nifL mut 22 for } & \text { T478A } & \text { CGCGACCGCAGGGAGGCGCCAGCCTGGTTCT } \\ & & \text { GCG } \\ \text { nifL mut 22 rev } & \text { T478A } & \text { CGCAGAACCAGGCTGGCGCCTCCCTGCGGTC } \\ & & \text { GCG } \\ \text { nifL mut 23 for } & \text { D353G } & \text { CAGCCCTTTTTTGGCGACCTGTACG } \\ \text { nifL mut 23 rev } & \text { D353G } & \text { CGTACAGGTCGCCAAAAAAGGGCTG }\end{array}$

Establishing a screening system to identify nif ${ }^{-}$mutants. The $\mathrm{pRS} 315^{*}$ pools containing the mutated nifL-genes were transformed into K. pneumoniae M5a1, separated on plates by selecting for kanamycin and ampicillin resistance and stored in 96 well microtiter plates. Single K. pneumoniae clones were grown in parallel under nitrogen fixing conditions on solid minimal medium and aerobically on LB as a control.

Mutants showing a nif ${ }^{-}$-phenotype under nitrogen fixing conditions were picked from the corresponding clones grown on LB medium. Subsequently each confirmed mutant was streaked out for single colonies from which 2 to 4 were again screened on solid medium.

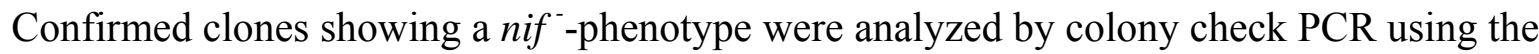
primer set nifL-T7-for and nifL-T7-rev (Tab. 4.2) in order to confirm the correct insert size of $1.7 \mathrm{~kb}$. Finally growth of nif-'-clones carrying a $1.7 \mathrm{kbp}$ was confirmed in liquid minimal medium with molecular nitrogen in the gas phase as sole nitrogen source.

Sequencing PCR positive clones. After confirming the proper insert size of the $1.7 \mathrm{kbp}$ fragment on plasmid pRS315* the DNA sequence of the respective mutated nifL-genes were independently determined for both strands in the Institut für Klinische Molekularbiologie (AG Prof. Dr. S. Schreiber, University of Kiel) using the primer sets M13 for/rev and the internal sequencing primer nifL-int-for (see Table 4.2).

Site directed mutagenesis. Site specific single mutations were introduced into nifL-gene of pRS315 using the QuickChange XL Site directed Mutagenesis Kit (Stratagene, La Jolla, USA) according to the protocol of the manufacturer and using the respective primer pairs as listed in Table 4.2. 


\section{Prediction of critical amino acid residues by analysis of $A$. vinelandii PAS1 domain of NifL.}

The structure of the FAD containing PAS1 domain of $A$. vinelandii NifL was illustrated using PyMOL тм v 0.99 (DeLano Scientific, LLC) providing the basis of predicting K. pneumoniae NifL amino acid residues which might be critical for membrane association (Key et al., 2007).

\section{Results}

We recently showed that under nitrogen fixing conditions the electrons from the reduced menaquinone pool are transferred to the FAD-cofactor of NifL in an unspecific way resulting in membrane sequestration of the negative regulator. Here we report on a general mutagenesis of nifL to identify single amino acid residues or surface region of NifL essential for membrane association.

Generating a pool of mutated nifL-gene. To construct pools of randomly mutagenized nifL we performed a saturated mutagenesis by PCR-amplification under low Taqpolymerase fidelity (see Materials and Methods). pJES283 containing the nifL-gene under control of the $\mathrm{T} 7$ promoter was used as template; to avoid mutations in the $T 7$ promoter the forward PCR-primer included the T7 promoter region (Fig. 4.1B) (see Materials and Methods). The amplification products were TA-cloned into the pCRII vector (Invitrogen), separated by transformation in E. coli and the resulting plasmids designated pRS315*. Three plasmid pools (RT1 - RT3) were generated independently consisting of approximately 12.000 mutated pRS315*. The mutation frequency of nifL in the plasmid pools determined by sequencing randomly chosen plasmids was in the range of 2 to 16 point mutations per nifL resulting in up to 9 parallel amino acid changes in the protein. 


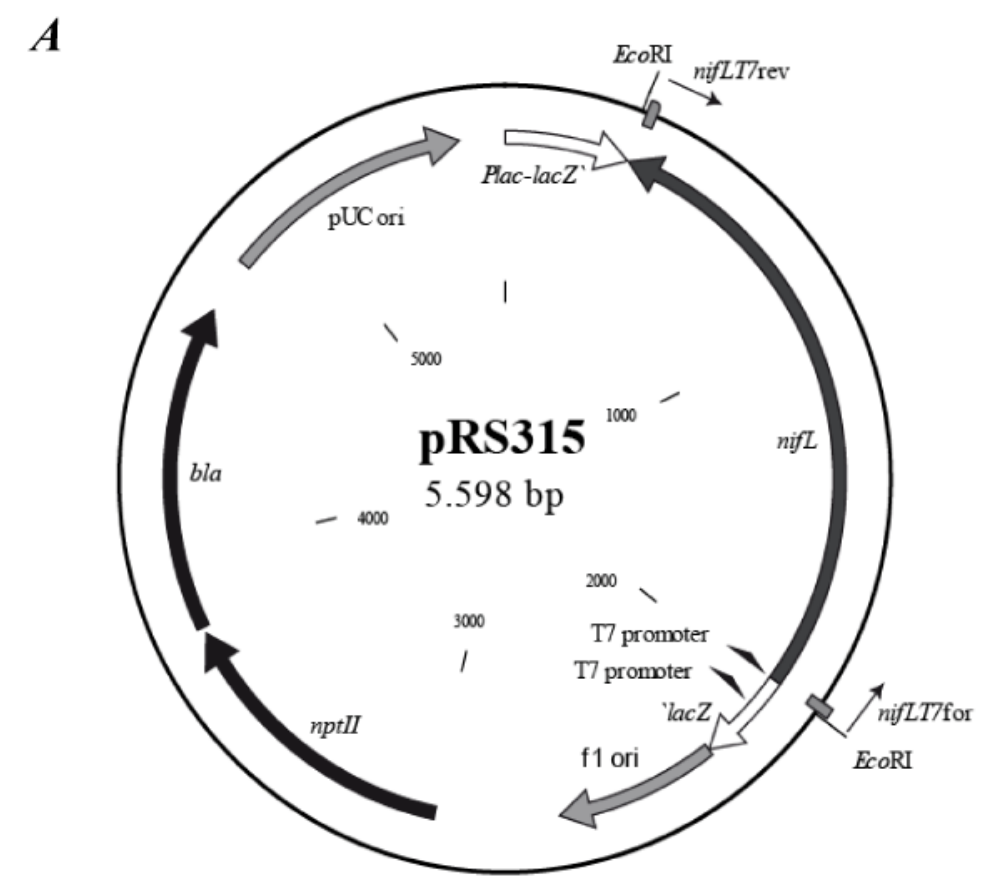

$\boldsymbol{B}$

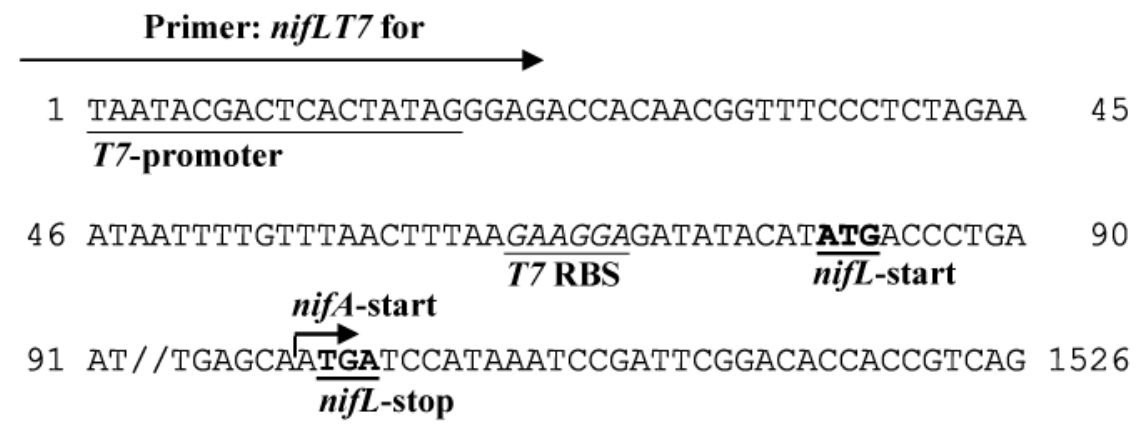

1527 ACGTTTCGATCTCTCCCAGCAGTTTA 1553

Primer: nifLT7 rev

Figure 4.1: Construction of pRS315. The nifL-gene was PCR amplified under high and low fidelity of the Taq-polymerase using pJES283 as template and the primer set nifLT7 forward/reverse. The $1.7 \mathrm{kbp}$ PCR-product was TA-cloned into pCRII TOPO (Invitrogen) and designated pRS315 for wild type nifL and pRS315* for mutated nifL.

A) Plasmid map of pRS315 B) Detailed section showing the location of the forward PCR primer overlaying the $T 7$ promoter region. The $T 7$ promoter region is underlined and the start and stop codons of nifL are marked in bold; besides the T7-ribosome binding site is shown in italics. The reverse primer is binding approximately $30 \mathrm{bp}$ after the stop codon of nifL in the nifA coding region.

Screening for nifL-mutants conferring a nif-phenotype under nitrogen fixing conditions. In order to identify NifL mutants which show a reduced membrane sequestration under nitrogen fixing conditions we transformed the respective constructed 
plasmid pools into K. pneumoniae wild type cells. Under nitrogen fixing conditions NifL expressed from the chromosomal nifLA-operon is completely sequestered to the cytoplasmic membrane. Additional expression of nifL wild type from the plasmid pRS315 slightly reduced the nitrogen fixation rate yielding in growth reduction of K. pneumoniae cells due to higher amounts of the negative regulator in relation to NifA (Fig. 4.2). In clones expressing mutated NifL from plasmids (pRS315*) and which showed a significantly reduced growth in comparison to K. pneumoniae/pRS315, plasmid encoded NifL* is apparently not completely sequestered to the cytoplasmic membrane. Thus, a certain amount of NifL* remained in the cytoplasm inhibiting cytoplasmic NifA resulting in a significantly reduced nitrogen fixation rate (Fig. 4.3C). NifL mutants with mutations in the C-terminal domain yielding in non-inhibitory NifL proteins are not detected in the screen as nitrogen fixation is not effected.

Initially approximately $11.500 \mathrm{~K}$. pneumoniae clones carrying pRS315* derived from 3 independently generated mutant pools were screened for growth on solid minimal medium under nitrogen fixing conditions (Fig. 4.3A) (see also Materials and Methods). K. pneumoniae wild type (nif ${ }^{+}$), UN4495 (nif-) and K. pneumoniae carrying pRS315 were analyzed as controls. During this initial screen 1203 mutants showed a nif--phenotype under conditions appropriate for nitrogen fixation. The nif ${ }^{-}$-phenotype of 495 clones was confirmed in a second screen on solid medium. (Tab. 4.3). Each of these confirmed mutant clones were streaked for single colonies and 2 to 4 colonies of each mutant were analyzed again for a nif - -phenotype on solid medium. In this screening step 313 clones respectively were confirmed to result a nif ${ }^{-}$-phenotype on solid minimal medium (Tab. 4.3). 


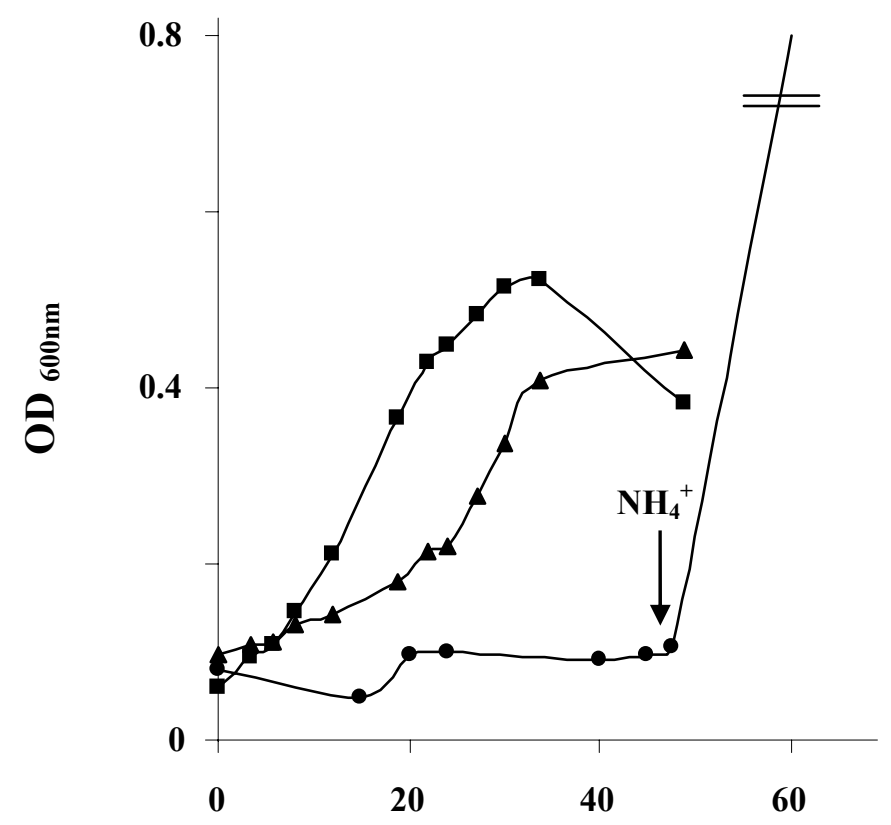

Time (h)

Figure 4.2: Screening system for nif -growth in liquid media under nitrogen fixing conditions. K. pneumoniae cells were grown under nitrogen and oxygen limitation with sucrose as carbon and energy source and molecular nitrogen as sole nitrogen source. To screen for nif phenotype the following strains were used as controls: K. pneumoniae wild type (M5a1; - ), K. pneumoniae carrying pRS315 (nifL under T7-control; $\mathbf{\Delta}$ ) and UN4495 (•) which is unable to fix molecular nitrogen. The additional expressed NifL in K. pneumoniae carrying pRS315 lead to reduced growth compared to the wild type. After approximately $48 \mathrm{~h} 10 \mathrm{mM}$ ammonium was added to the UN4495 (indicated by an arrow).

To avoid false positive clones which e.g. carry only the C-terminal domain of NifL, which is sufficient to inhibit NifA under all conditions, we analyzed the 313 plasmids by colonycheck PCR using the primers nifLT7-forward und nifLT7-reverse (see also Materials and Methods). Interestingly RT1 and RT2 exhibited a much higher number of mutants which were not carrying the complete nifL-gene compared to RT3. A total of approximately $33 \%$ of mutants conferring a nif ${ }^{-}$-phenotype on solid medium showed inserts smaller than $1.7 \mathrm{kbp}$. Sequence analysis of 8 randomly chosen plasmids with an insert size smaller than $1.7 \mathrm{kbp}$ indicated that the central region of the gene was deleted resulting in mutants carrying the inhibitory $\mathrm{C}$-terminal part of the protein but lacking parts of the redox sensing N-terminal domain and thus yielding in a nif - ${ }^{-}$phenotype (Tab. 4.3). 73 clones (RT1 + RT2) and 134 clones (RT3) which showed a DNA fragment of accurate size corresponding to nifL were further characterized by growth analysis in liquid medium under nitrogen fixing conditions. In this screen we identified 4 different types of mutant phenotypes to which the 207 positive clones after confirmation of the $1.7 \mathrm{kbp}$ DNA fragment by colonycheck PCR 

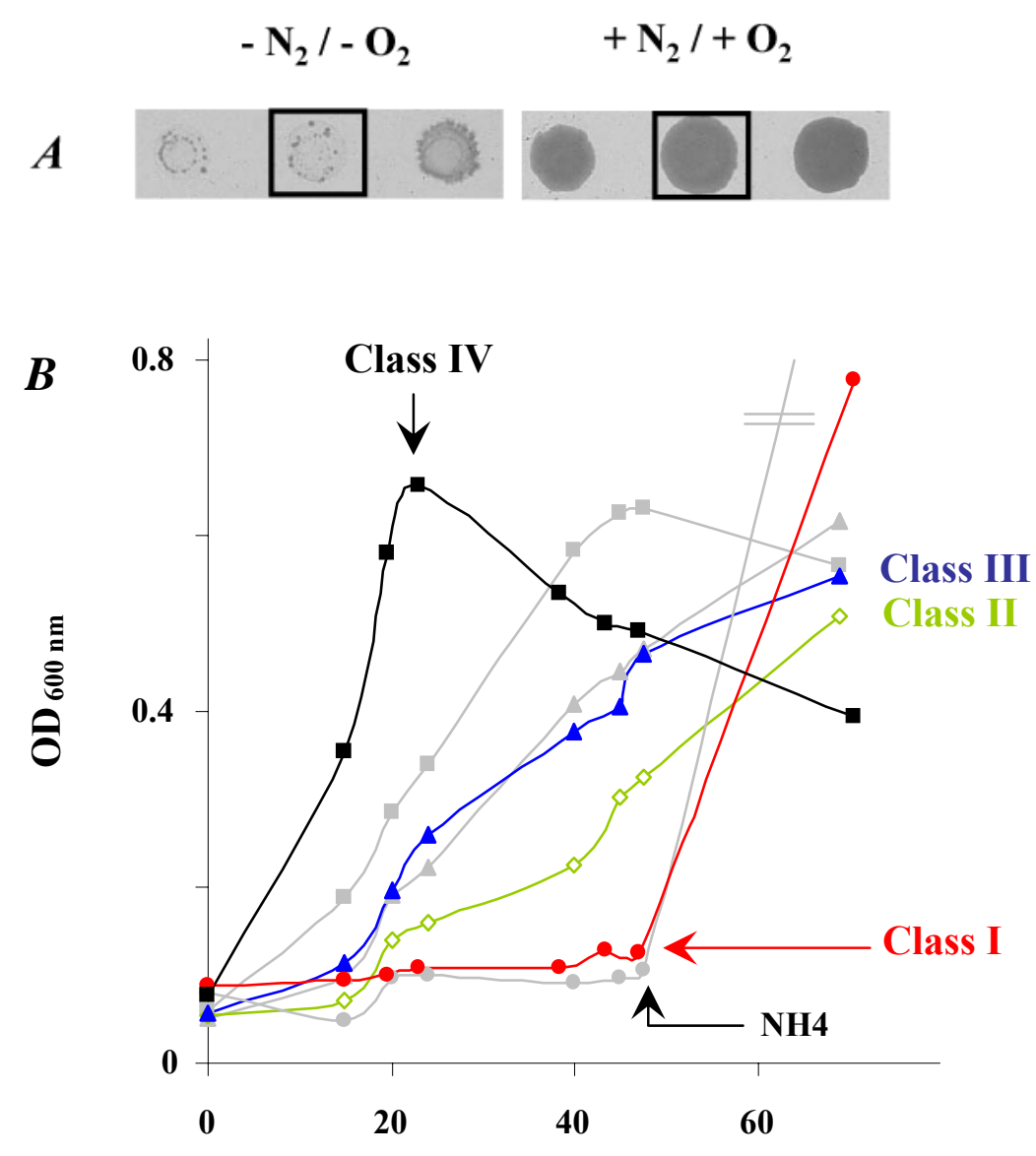

Time (h)

Figure 4.3: Screening nifL-mutants on solid medium and in liquid medium. Mutant clones (K. pneumoniae/pRS315*) were screened on solid medium under nitrogen fixing conditions (A, left panel) and in parallel on LB medium (A, right panel). Clones conferring a nif -phenotype (indicated boxed) were validated in another screening step and single colonies of confirmed mutants were analyzed.

B) Single colonies showing a nif -phenotype on solid medium were grown in $25 \mathrm{ml}$ minmal medium under nitrogen fixing conditions with molecular nitrogen as sole nitrogen source. Four different classes of mutants were defined and are shown here with red, green, blue and black lines: Class I, no growth $(\bullet)$; class II, decreased growth compared to K. pneumoniae/pRS315 ( $\diamond)$; class III growth in the range of K. pneumoniae/pRS315 and K. pneumoniae wild type ( $\Delta$ ); class IV, increased growth compared to K. pneumoniae wild type ( $\square)$. Control strains, M5a1 ( $\square$ ); K. pneumoniae/pRS315 ( $\triangle)$; UN4495 ( $\bullet$ ).

were classified. (i) Class I mutants showing a severe nif - - phenotype (growth comparable to the negative control UN4495), (ii) class II mutants growing slightly faster than UN4495, (iii) clones growing in the range of K. pneumoniae wild type carrying pRS315 (positive control; class III) and (iv) class IV mutants showing even a faster growth than K. pneumoniae wild type (Fig. 4.3B). After approximately $50 \mathrm{~h}$ incubation at $30^{\circ} \mathrm{C} 10 \mathrm{mM}$ 
ammonium was added to the cultures to exclude secondary mutations and demonstrate viability of the clones. The results of this screening step and the classification of the positive clones are summarized in Table 4.3. After this intensive screening procedure about $0.5 \%($ RT $1+2)$ and $0.7 \%$ (RT3) of all clones initially tested showed a nif - - phenotype. Sequence analysis of plasmids carrying the mutated nifL-gene revealed that 1 to 9 amino acid mutation(s) per NifL protein were introduced resulting in a significant phenotype. Besides mutations in the N-terminal domain, interestingly, approximately $10 \%$ of nifLmutants showing a nif - ${ }^{-}$phenotype carried mutations exclusively in the $\mathrm{C}$-terminal domain (Fig. 4.5) and one mutant appeared to carry one single amino acid exchange within the Qlinker region of NifL.

Table 4.3: Progress of the screening system

\begin{tabular}{|c|c|c|c|c|}
\hline Pool & $\begin{array}{l}\text { No. of } \\
\text { clones }\end{array}$ & Screeening step & $\begin{array}{c}\text { Clones with nif - } \\
\text { phenotype }\end{array}$ & $\begin{array}{c}\text { Rate of analyzed } \\
\text { clones }(\%)\end{array}$ \\
\hline $\mathrm{RT} 1+2$ & 5760 & $1^{\text {st }}$, solid medium & 648 & 11.25 \\
\hline RT 3 & 5760 & & 555 & 9.6 \\
\hline $\mathrm{RT} 1+2$ & & $2^{\text {nd }}$, solid medium & 170 & 3 \\
\hline RT 3 & & & 325 & 5.6 \\
\hline $\mathrm{RT} 1+2$ & & $3^{\mathrm{rd}}$, solid medium, & 155 & 2.7 \\
\hline RT 3 & & single colonies & 158 & 3.7 \\
\hline RT $1+2$ & & colony check PCR & 73 & 1.3 \\
\hline RT 3 & & & 134 & 2.3 \\
\hline $\mathrm{RT} 1+2$ & 73 & & 12 (class I) & 0.21 \\
\hline & & liquid medium & 15 (class II) & 0.26 \\
\hline RT 3 & 134 & & $\begin{array}{l}14 \text { (classI) } \\
26 \text { (classII) }\end{array}$ & $\begin{array}{l}0.24 \\
0.45\end{array}$ \\
\hline
\end{tabular}


Selection of amino acids for separation by site-directed mutagenesis and construction of single mutations. In order to identify single amino acid residues which might be critical for membrane association of NifL the FAD containing PAS1 domain of $A$. vinelandii for which a crystal structure is available (Key et al., 2007) was compared with K. pneumoniae NifL for which the structure is unknown at present. Those amino acid residues were separated which (i) coordinate the FAD-cofactor in A. vinelandii NifL, (ii) are likely to reside near the FAD-binding site and finally (iii) are predicted to be located on the protein surface potentially involved in contacting the cytoplasmic membrane. Additionally we evaluated which amino acid residues were frequently changed in different mutants in the general screen. Mutated amino acid residues which were predicted to belong to one of the groups indicated above were chosen for separation (Tab. 4.2).

The single amino acid exchange was performed using the plasmid pRS315 as template for the site-directed-mutagenesis using the respective primers (Tab. 4.2). After confirming the introduced mutation by sequencing the plasmid was transformed into K. pneumoniae M5a1 and the nif ${ }^{-}$-phenotype was determined in liquid medium under nitrogen fixing conditions. Only 2 out of 20 mutants investigated (R80C and Q57L) showed a nif--phenotype (Fig. 4.4). 
A

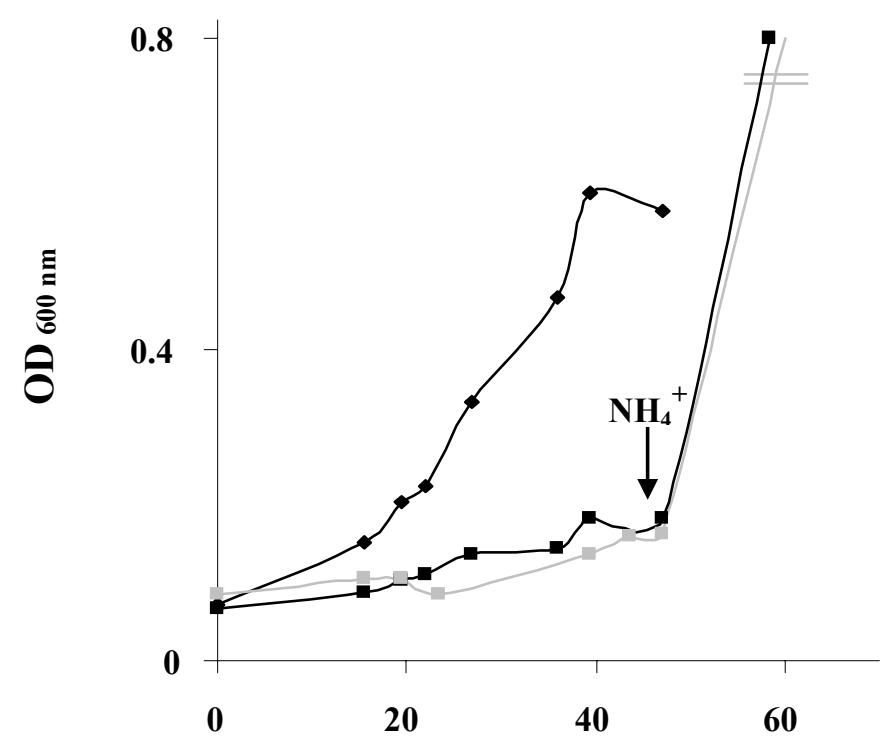

Time (h)

$\boldsymbol{B}$

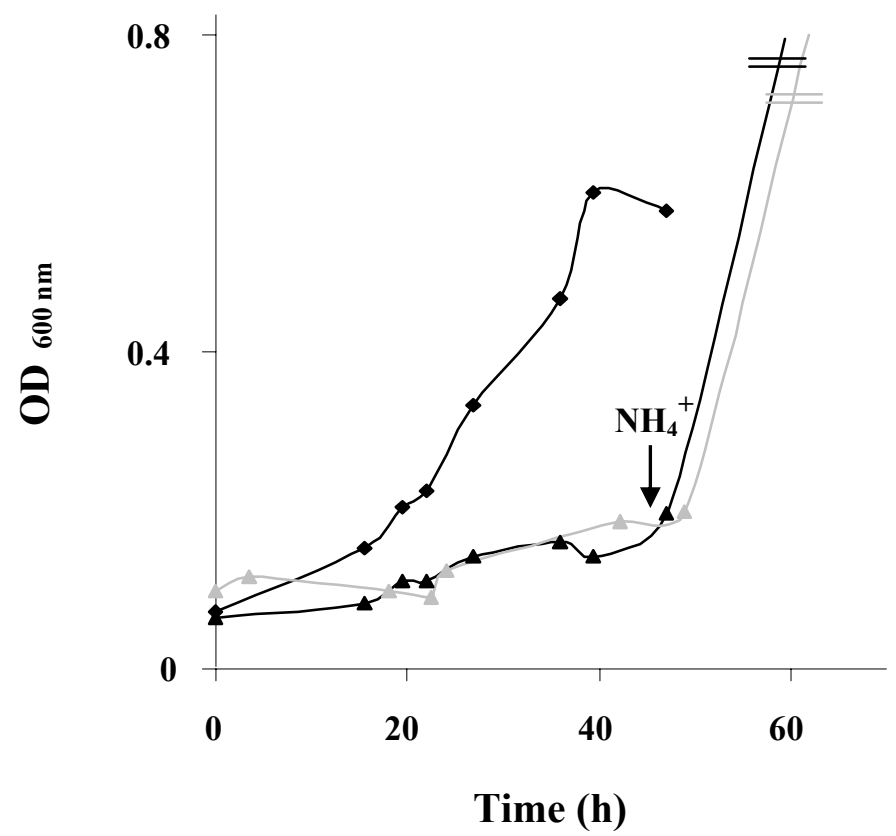

Figure 4.4: Growth of K. pneumoniae carrying NifL R80C and Q57L on a plasmid. Growth of clones carrying single amino acid mutations under nitrogen fixing conditions was analyzed in comparison with originally identified multiple mutants and control strain K. pneumoniae/pRS315.

A) K. pneumoniae/pRS315* (R80C, Q88R; $)^{*}$ is depicted as gray line with gray squares. Black line with black squares show $K$. pneumoniae/pRS315* (R80C, clone I; - ). Control strain K. pneumoniae/pRS315* is indicated as black line with black rhombs $(\bullet)$. 
B) Gray line and gray triangles indicate multiple mutant K. pneumoniae/pRS315* (Q57L, V119A; $\triangle$ ), K. pneumoniae/pRS315 (Q57L, clone II; $\Delta$ ) is depicted as black line with black squares (clone II). K. pneumoniae/ pRS315 as control is shown as black line with black rhombs (•).

Besides one of the multiple mutants with a class I nif carrying exclusively mutations in the C-terminal domain was further analyzed. Therefore 3 single amino acid mutations were introduced to pRS315 (R335G, D353G and Q478A) and growth of the respective mutants was monitored (Fig. 4.5). Interestingly, in contrast to the original multiple mutant all three single amino acid mutations indicated a class II phenotype.

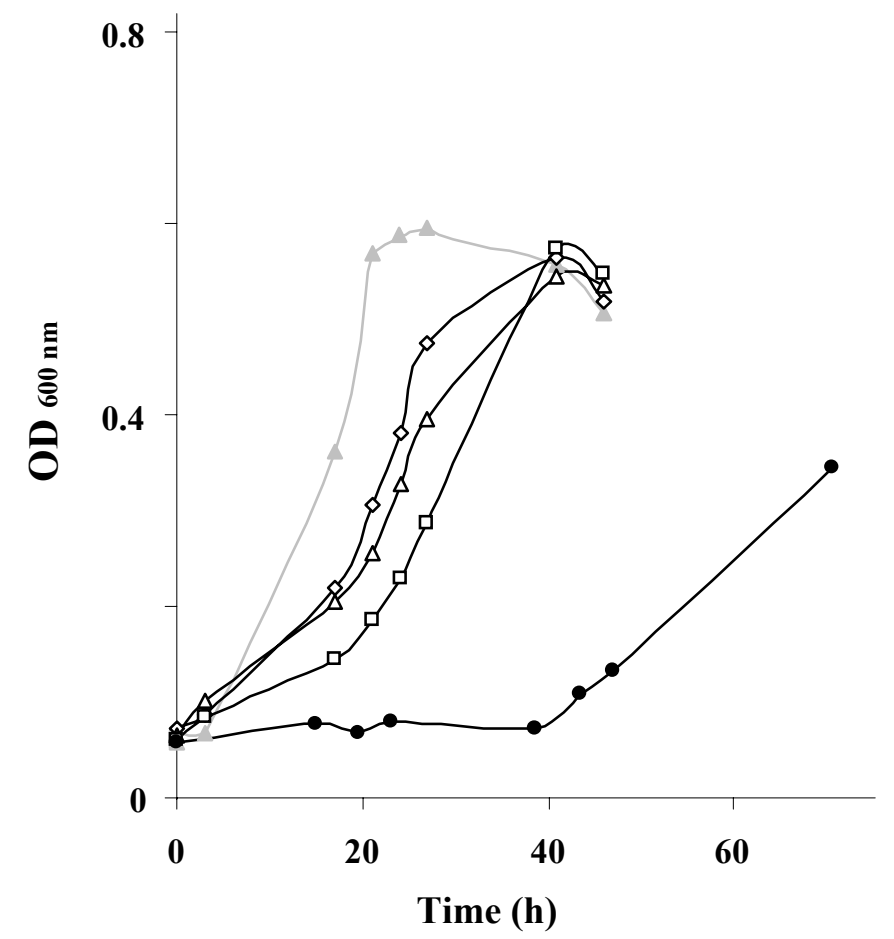

Figure 4.5: Growth analysis of NifL*-mutants carrying mutations in the C-terminal domain. Growth of clones carrying single amino acid mutations located in the C-terminal domain of NifL compared to the respective multiple mutant and control strain K. pneumoniae/pRS315 under nitrogen limitation.

K. pneumoniae/pRS315* (R335G; $\diamond),(\mathrm{D} 353 \mathrm{G} ; \square)$ and $(\mathrm{N} 478 \mathrm{~A} ; \Delta)$ and the respective originally identified multiple mutant $(\bullet)$ are depicted as black lines. Control strain K. pneumoniae/pRS315 is marked with a gray line and gray triangles $(\triangle)$ 


\section{Discussion}

Nitrogen fixing organisms need to tightly control transcription and activity of nitrogenase in response to the environmental signals oxygen and nitrogen. In order to avoid unnecessary consumption of energy nif - gene expression occurs exclusively in the absence of combined nitrogen under simultaneous anaerobic conditions (Burgess and Lowe, 1996; Halbleib and Ludden, 2000; Howard and Rees, 1996; Rees and Howard, 1999). Under these conditions the transcriptional activator NifA is not inhibited by its negative regulator NifL and activates transcription of nif-genes (Hoover et al., 1990; Morett and Buck, 1989; Ray et al., 2002). This regulation is based on NifL sequestration to the cytoplasmic membrane under appropriate conditions for nitrogen fixation (Klopprogge et al., 2002; Stips et al., 2004). In this study a PCR based mutagenesis of nifL was performed to obtain mutants which show a nif--phenotype under nitrogen fixing conditions.

Architecture of NifL. The negative regulator NifL consists of an N-terminal redox sensing domain (amino acids 1 to 255) carrying an FAD containing PAS domain, a C-terminal domain (amino acids 281 to 495) interacting with NifA and a Q-linker which is a hydrophilic interdomain linker connecting N-terminal and C-terminal domains located between amino acid residues 256 and 280 (Fig. 4.6 A). Most of the mutants obtained in this study conferring a nif -phenotype showed mutations in the N-terminal domain. Three mutants were identified carrying mutations exclusively in the C-terminal domain and one mutant was showing one single amino acid mutation in the Q-linker region.

Structure of the PAS1 domain of A. vinelandii NifL was used to predict critical amino acid mutations. The crystal structure of K. pneumoniae NifL has not been solved yet, however, the structure of the N-terminal FAD containing PAS domain of $A$. vinelandii (amino acids 11 to 137 ) is available (Key et al., 2007). As the K. pneumoniae and A. vinelandii NifL shows approximately $40 \%$ homology and approximately $60 \%$ similarity concerning the PAS domain on the amino acid level (Fig. 4.6 B) the known structure was used to predict the position of obtained amino acid exchanges resulting in a nif -phenotype. Mutations presumably involved in either FAD binding or located on the surface and thus potentially contacting the cytoplasmic membrane were chosen to be separately introduced into nifL wild type by site directed mutagenesis. The structure of $A$. vinelandii NifL revealed amino 
A

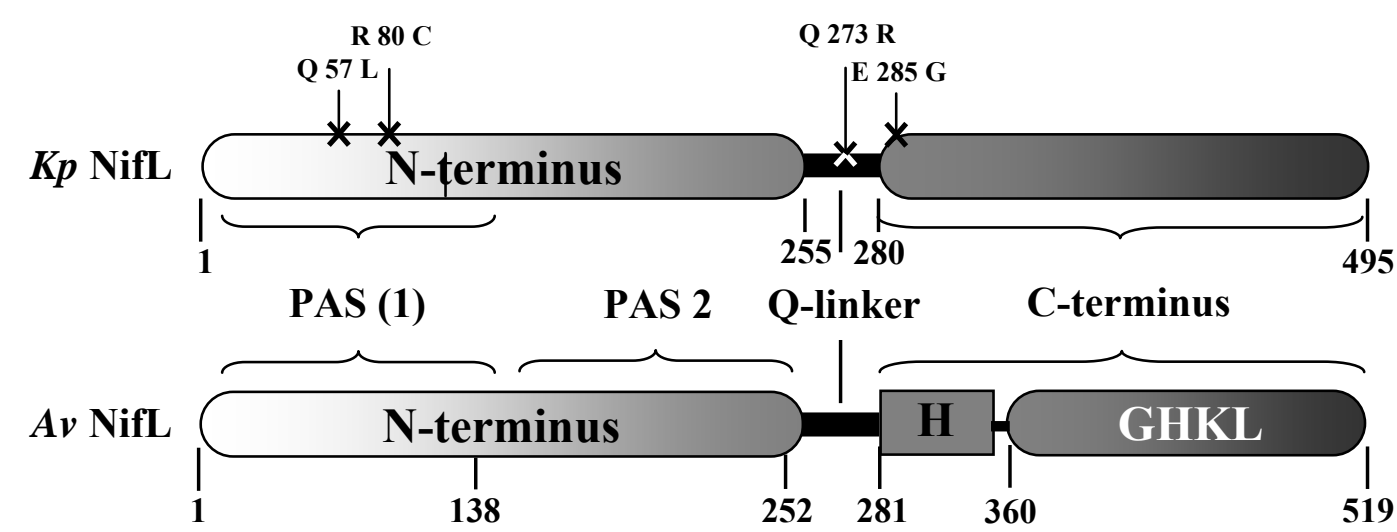

$\boldsymbol{B}$

Kp 1 MTLNMMLDNAVPEAIAGALTQQHPGLFFTMVEQASVAISLTDARANI IYANPAFCRQTGY MT $\quad \mathrm{P}+\mathrm{A}+\mathrm{P}+\mathrm{F}$ VE A +AIS+TD +ANI+YAN AF TGY AV 1 MTPANPTLSNEPQAPHAESDELLPEIFRQTVEHAPIAISITDLKANILYANRAFRTITGY 60

61 SLAQLLNQNPRLLASSQTPREIYQEMWQTLLQRQPWRGQLINQRRDGGLYLVDIDITPVL $++\mathrm{L}+\mathrm{N}+\mathrm{L}++$ TPR $+\mathrm{YQ}+\mathrm{W} \mathrm{L}$ Q++PW G L+N+R+D LYL ++ + PVL

61 GSEEVLGKNESILSNGTTPRLVYQALWGRLAQKKPWSGVLVNRRKDKTLYLAELTVAPVL

120

121 NPQGELEHYLAMQRDISVSYTLEQRLRNHMTLMEAVLNNIPAAVVVVDEQDRVVMDNLAY $\mathrm{N}$ GE +YL M RD S + LEQR+ N ++EAV+N PAA+VV+D Q RV++ N ++

121 NEAGETIYYLGMHRDTSELHELEQRVNNQRLMIEAVVNAAPAAMVVLDRQHRVMLSNPSF 120

180 191 KTFCADC - - - GGKELLVEL - - - - - - - - QVSPRKMGPGAEQILPVVVRGAVRWLSVTCW

ALPGVSEEASRYFVDSAPARTLMVIADCTQQRQQQEQGRLDRLKQQMMTAGKLLAAIRESL $\mathrm{A}+\quad+\mathrm{E}+\mathrm{A}+\mathrm{F}+\mathrm{L}+\mathrm{I} \mathrm{D}++\mathrm{RQ}+\mathrm{Q}+\mathrm{RL}+\mathrm{LK} M+\mathrm{ML}+\mathrm{R} \overline{\mathrm{E}}+$

DAALIQLNCPINMLAAARRL - - - - NGEGSGNVALDAAWRE - - - - GEEAMARLQRCRPSLE $+\mathrm{AA}++\mathrm{L} \quad \mathrm{P}+\mathrm{N}+++\mathrm{AA} \mathrm{R}+\quad \mathrm{G}++\mathrm{GN}++\mathrm{A} \mathrm{RE} \quad \mathrm{G} \mathrm{EA}+\mathrm{L}{ }_{\mathrm{P}}$

301 NAAIHRLQGPVNLISAAMRMLERRLGDKAGNDPVLSAMREASTAGMEALENLSGSIPVRM 371

340 LESNAVWPLQPFFDDLYALYRTRFDDRARL - - QVDMASPHLVGFGQRTQLLACLSLWLD $\mathrm{ES} \quad \mathrm{L}++\mathrm{L}+++\mathrm{Q}+\mathrm{P}++\mathrm{G}++\mathrm{Q}+++\mathrm{D}$

372 AESKMPVNLNQLIREVITLCTDQLLAQGIVVDWQPALRLPWVMG-GESSQ-RSMIKHLVD 429

397 RTLALAAELPSVPLEIELYAEEDEGWLSLYLNDN - - - VPLLQVRYAHSPDALNSP - - - - G 449

$$
+\quad++\quad \mathrm{E}++\quad+++\mathrm{D}+\quad+\mathrm{P} \quad \mathrm{V} \quad \mathrm{P} \quad \mathrm{P} \quad \mathrm{G}
$$

430 NAIESMSQNQVSRRELFISTRVENHLVRMEITDSGPGIPPDLVLKVFEPFFSTKPPHRVG 489

450 KGMELRLIQTLVAYHRGAIELASRPQGGTSLVLRFPLFNTLTGGEQ 495 $+\mathrm{GM} \mathrm{L}++\mathrm{Q}+\mathrm{VA} \mathrm{H} \mathrm{G}++++\mathrm{G}^{-}+\mathrm{V}+\mathrm{P}$

$49 \odot$ RGMGLPVVQEIVAKHAGMVHVDTDYREGCRIVVELPFSAST

Figure 4.6: Domain structure and amino acids of $K$. pneumoniae and $A$. vinelandii NifL. A) K. pneumoniae NifL and A. vinelandii NifL show high homology in the N-terminal domain containing the PAS domain(s). The C-terminal domain, however, is less homologous on amino acid level. Confirmed single amino acid mutations conferring a nif ${ }^{-}$-phenotype are marked with an $\mathrm{x}$ in 
the K. pneumoniae NifL cartoon which is modified from Narberhaus et al. (Narberhaus et al., 1995). The A. vinelandii NifL cartoon is modified from Little et al. (Little et al., 2007).

B) Alignement of $K$. pneumoniae and A. vinelandii NifL. The amino acid residues which coordinate the FAD-cofactor of $A$. vinelandii and the respective conserved amino acid residues of K. pneumoniae are marked with light grey bars. Confirmed single amino acid residues of K. pneumoniae NifL which result in a nif - -phenotype when mutated are underlined and marked in bold.

acid residues 78 to 88 forming an $\alpha$-helix and residues 69, 70 and 102, are presumably required to directly coordinate the FAD-cofactor (Fig. 4.7) (Key et al., 2007). Two of the amino acids within the $\alpha$-helix, R80 and W87, and residues N69, E70 and N102 form hydrogen bonds with the FAD and except E70 these residues are conserved in A. vinelandii NifL and K. pneumoniae NifL (Fig. 4.6 B). Mutations in this areas would probably lead to NifL apoenzyme which can not be reduced and associated to the cytoplasmic membrane under anaerobic conditions resulting in cytoplasmic NifL inhibiting NifA activity.

Mutated amino acid residues which are not situated near the FAD binding site causing a nif ${ }^{-}$-phenotype were expected to be located on the protein surface and are likely to be involved in contacting the cytoplasmic membrane. One can speculate that mutations of amino acid residues which are required to sequester NifL to the membrane also result in cytoplasmic NifL under nitrogen fixing conditions. Consulting the structure of A. vinelandii NifL we identified 16 mutants which presumably are located on the protein surface and four which were predicted to be located near the FAD binding site.

Mutations potentially affecting FAD coordination. Three of four mutations which were predicted to be located near the FAD binding site of NifL were separated by site directed mutagenesis and the nif -phenotype (class I) was confirmed for amino acid exchange R80C strongly indicating that amino acid residues R80 plays a crucial role in oxygen signaling. As the original multiple mutant obtained in the screen carries only one additional mutation it is very likely that R80 is responsible for the nif - ${ }^{-}$phenotype of the clone. This is further verified by the fact that the second mutation of the original mutant carrying R80C was identified to be Q88R. This mutation did not show an effect on the phenotype after separation clearly indicating that $\mathrm{R} 80 \mathrm{C}$ is sufficient to interrupt oxygen signaling. This might be in accordance to findings for $A$. vinelandii NifL for which the arginine at position 80 has been shown to be one of the amino acids coordinating the FAD (Fig. 4.7) (Key et al., 2007). 


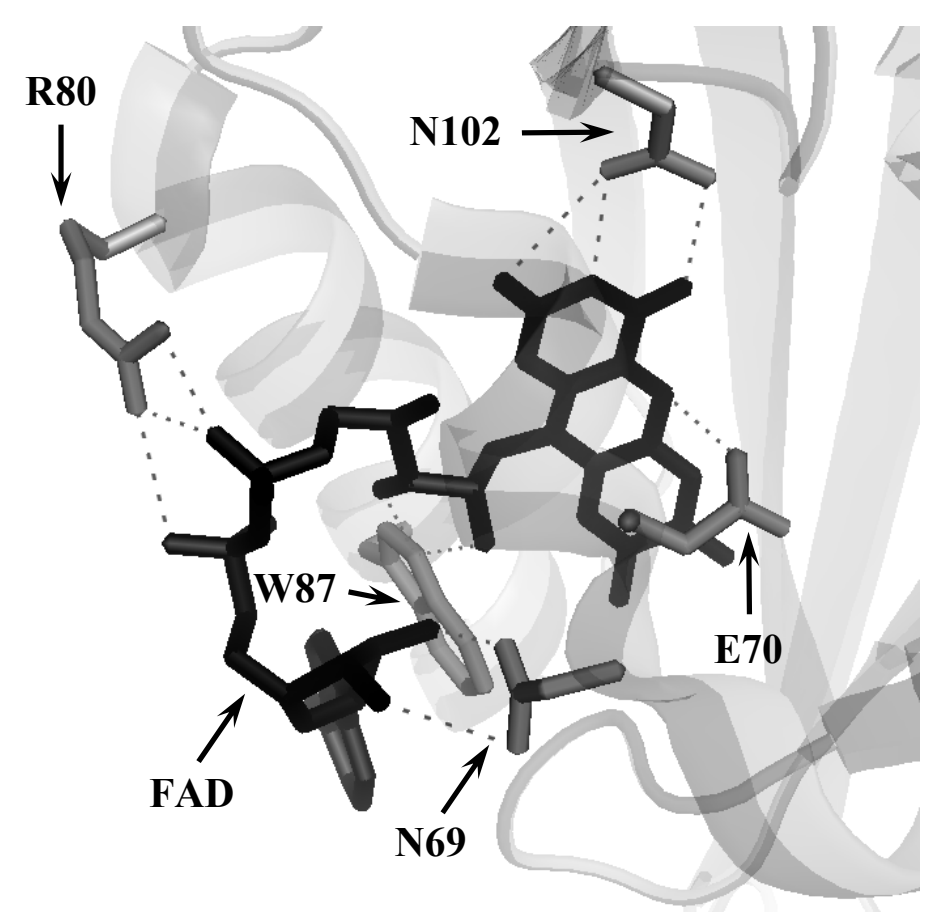

Figure 4.7: Section of the PAS1 domain of A. vinelandii NifL structure. The section of A. vinelandii NifL structure shows part of the FAD-cofactor containing PAS1 domain (Key et al., 2007). The amino acid residues directly coordinating the FAD-cofactor by forming hydrogen bonds are indicated by arrows and position and amino acid are presented.

Mutations of NifL amino acid residues located at the protein surface. Overall 16 mutated amino acid residues have been identified to be presumably located on the protein surface. After separation of the respective amino acid mutations the nif - - phenotype (class I) has been confirmed for a mutant carrying mutation Q57L. The original multiple mutant obtained during screening process offered one additional mutation which was shown to be V119A. Because Q57L is sufficient to prevent K. pneumoniae of fixing molecular nitrogen resulting in a nif - -phenotype under nitrogen fixing conditions and due to the fact that V119 seems to be neither located on the protein surface nor in the near of the FAD binding site this amino acid residue potentially does not to play a role in oxygen signaling. In contrary it is very likely that residue 57 is involved in contacting cytoplasmic membrane and thus the mutation at this position results in cytoplasmic inhibitory NifL. This finding has to be further supported by constructing a single amino acid mutation at position V119. An effect on membrane contact of this mutation has to be shown by membrane preparation analysis. 
Mutations conferring a nif -phenotype located in the Q-linker region of NifL. Interestingly Clone XXV (Tab. 4.1) exclusively carries a mutation within the Q-linker at position 273 resulting in a class II phenotype. Austin and coworkers suggest that in A. vinelandii this Qlinker probably is involved in conformational changes of NifL resulting in complex formation with NifA (Money et al., 2001). Although in K. pneumoniae the Q-linker is not required for NifA inhibition (Narberhaus et al., 1995) the observation that a single amino acid mutation in this region results in a decreased growth rate of the mutant strain under conditions appropriate for nitrogen fixation lead one to assume that the Q-linker of K. pneumoniae plays a certain role in NifL conformation. Mutations in this region probably destabilize inhibitory NifL/NifA complexes or prevent the protein from associating to the cytoplasmic membrane.

C-terminal mutations resulting in a nif-phenotype. Unexpectedly we also received mutants carrying amino acid mutations located exclusively in the C-terminal domain of NifL. In addition to clone XXIV which bears one single mutation close to the Q-linker at position 285 (E285G) potentially also effecting conformation of NifL (discussed below), two more mutants were identified carrying solely C-terminal mutations. One of the original multiple mutants showing a class I phenotype (no growth) in liquid minimal medium was selected to further investigate which amino acid residues of NifL might be involved in binding to NifA resulting in a "super-inhibiting" NifL mutant. Three out of five amino acid mutations were separated (R335G, D353G and T478A) and growth under nitrogen fixing conditions has been monitored revealing that all three single mutations show a decrease in the growth rate of K. pneumoniae but no mutation seems to be exclusively responsible for the severe nif ${ }^{-}$-phenotype of the original mutant (Fig. 4.5). For A. vinelandii it has been demonstrated that mutations in the C-terminal domain resulting in relief of NifA show decreased binding affinity to ADP. Binding of adenosine nucleotides increases the inhibitory effect of NifL on NifA-activity (Perry et al., 2005). In contrary nucleotide binding has no effect on NifL mediated regulation of NifA in K. pneumoniae but direct protein-protein interaction is the exclusive mechanism for regulation (Govantes et al., 1998; Henderson et al., 1989; Stips et al., 2004). Therefore the findings obtained here indicate that the amino acid residues R335, D353 and T478 are either jointly responsible for a NifL conformation allowing NifL to form highly inhibitory complexes with NifA or directly interact with the transcriptional activator both resulting in a highly increased stability of NifL/NifA complexes. 
Mutations in the C-terminal domain corresponding to A. vinelandii $H$ domain. In A. vinelandii it has been demonstrated that mutations in the $\mathrm{C}$-terminal $\mathrm{H}$ domain of NifL lead to proteins which are not able to properly regulate NifA activity upon the oxygen signal or even both nitrogen and oxygen signal. These findings indicate that the $\mathrm{H}$ domain of NifL is required for relaying the oxygen and the nitrogen signal to influence the interaction with NifA (Little et al., 2007). In addition to multiple mutants with mutations also in the region of $K$. pneumoniae NifL corresponding to the $\mathrm{H}$ domain of $A$. vinelandii one single mutation at position 285 (E285G) was obtained. Although E285 is conserved in K. pneumoniae and A. vinelandii (E298) this residue has not been demonstrated to be critical for nitrogen or oxygen signaling in A. vinelandii (Little et al., 2007). Due to the fact that no further single mutations have been identified exclusively in the respective region of $K$. pneumoniae NifL and due to the fact that the $\mathrm{H}$ domain of $A$. vinelandii NifL does not show high homology to K. pneumoniae NifL it remains unclear whether this region also plays an important role in regulating NifA activity in K. pneumoniae. Probably it is more likely that the resulting nif - ${ }^{-}$phenotype of E285G results from the adjecency to the Q-linker region which potentially is necessary for keeping NifL in a conformation which allows regulating NifA-activity in response to environmental signals.

Conclusion. With this work we demonstrated that the majority of randomly introduced mutations into K. pneumoniae NifL, resulting in a nif ${ }^{-}$-phenotype under nitrogen fixing conditions, are located in the N-terminal redox sensing PAS domain. Separation of mutated amino acid residues resulted in two amino acid mutations which are apparently sufficient to inhibit NifA-activity as revealed by growth experiments in minimal medium. One amino acid mutation $(\mathrm{Q} 57 \mathrm{~L})$ is predicted to be located on the protein surface probably interfering membrane contact of the protein whereas the second mutation, $\mathrm{R} 80 \mathrm{C}$, is presumably involved in coordinating the redox sensitive flavin moiety of NifL which is in accordance to findings in A. vinelandii. As three more conserved amino acid residues coordinate the FAD-cofactor in A. vinelandii NifL it has to be further investigated whether mutations of these residues also result in constitutively inhibiting NifL* mutants. Besides additional amino acid residues situated on the protein surface have to be mutated in order to rule out which amino acids are involved in contacting the cytoplasmic membrane. Further biochemical studies have to demonstrate whether confirmed mutations result in a change of NifL localization under nitrogen fixing conditions. For that reason membrane preparations of different mutants have to be performed. To verify that amino acid mutations at residues 
which are predicted to coordinate the FAD-cofactor result in NifL apoenzyme yielding in a nif - ${ }^{-}$phenotype the amount of FAD in those mutants has to be determined compared to NifL holoenzyme. 
Chapter 5:

\section{Conclusion}

In nitrogen fixing organisms the transcription of nitrogen fixation (nif)-genes has to be strictly controlled to prevent unnecessary consumption of energy (Burgess and Lowe, 1996; Howard and Rees, 1996). In the free living diazotrophs K. pneumoniae and A. vinelandii the activity of the transcriptional activator NifA is modulated by the regulatory protein NifL in response to the environmental signals oxygen and nitrogen limitation (Dixon, 1998; Filser et al., 1983; Schmitz et al., 2002). Different studies revealed that the inhibition of NifA by NifL results from direct protein-protein interactions (Govantes et al., 1998; Henderson et al., 1989; Lei et al., 1999; Money et al., 1999; Money et al., 2001). Under conditions inappropriate for nitrogen fixation both proteins form an inhibitory complex impairing NifA to contact and activate the alternative $\sigma^{54}$ RNApolymerase thus inhibiting nif-gene expression (Hoover et al., 1990; Morett and Buck, 1989).

The nitrogen signal is transduced towards the NifL/NifA regulatory system by a small regulator, the PII-like protein, GlnK which is uridylylated/deuridylylated in response to the internal nitrogen status of the cell. (Atkinson and Ninfa, 1999; He et al., 1998; Jack et al., 1999; van Heeswijk et al., 1996; Xu et al., 1998). Although GlnK transduces the nitrogen signal to the nif-system in K. pneumoniae and A. vinelandii there are apparently species specific differences in the mechanism of nitrogen signal transduction. In A. vinelandii it has been shown that non-modified GlnK binds to the C-terminal domain of NifL enforcing the inhibitory function of NifL (Little et al., 2000; Little et al., 2002; Reyes-Ramirez et al., 2000). On the contrary for K. pneumoniae it has been demonstrated that GlnK is required for activating the nif-gene expression under nitrogen fixing conditions irrespective of its modification state (Arcondeguy et al., 1999; He et al., 1998; Jack et al., 1999). Although uridylylation of GlnK apparently has no influence on the relief of NifL inhibitory function (Arcondeguy et al., 1999; He et al., 1998) presumably only uridylylated GlnK stays in the cytoplasm under nitrogen limiting conditions thus allowing the NifL-NifA complex to dissociate. Merrick and coworkers have obtained evidence that non-uridylylated E. coli GlnK is sequestered from the cytoplasm to the cytoplasmic membrane in an AmtB dependent manner (Coutts et al., 2002). Very recently, crystal structures of E. coli GlnK 
confirmed that only the non-modified form of GlnK binds to AmtB thereby blocking the ammonium channel (Conroy et al., 2007).

The transduction of the oxygen signal towards NifA is mediated by the flavoprotein NifL in K. pneumoniae and A.vinelandii (Hill et al., 1996; Little et al., 2000; Schmitz, 1997; Soderback et al., 1998). Although in both organisms the N-terminal FAD moiety of NifL is reduced under anaerobic conditions, there are again species specific differences in sensing the oxygen signal. In $A$. vinelandii NifL is located in the cytoplasm independently of environmental conditions. Electrons for FAD reduction appear to be derived from intracellular reducing equivalents which are generated during growth under anoxic conditions (Dixon, 1998; Macheroux et al., 1998). It is suggested that a conformational change in the $\mathrm{H}$ domain of NifL relayed by the oxygen (signal reduction of the FADcofactor) results in dissociation of the inhibitory NifL/NifA complex due to a switch from inhibitory form of NifL to the non-inhibitory form (Little et al., 2007; Martinez-Argudo et al., 2004a, b).

In K. pneumoniae the reduction of the NifL bound FAD-cofactor occurs differently. Under conditions appropriate for nitrogen fixation the inhibitory NifL/NifA complexes dissociate and NifL is reduced at the cytoplasmic membrane by electrons derived from the reduced menaquinone pool (Grabbe et al., 2001; Grabbe and Schmitz, 2003; Klopprogge et al., 2002; Thummer et al., 2007). Upon electron transfer onto FAD the reduced NifL protein stays associated to the cytoplasmic membrane resulting in transcriptional active NifA. Thus the localizational change of NifL apparently is the key regulatory mechanism resulting in nif-gene expression in $K$. pneumoniae which clearly differs from the mechanism of conformational changes observed for $A$. vinelandii (Klopprogge et al., 2002).

The focus of the present thesis was to further investigate signal transduction of both environmental signals, nitrogen availability and absence of oxygen, onto the NifL/NifA regulatory system in K. pneumoniae. Therefore the interaction between the three regulatory proteins NifL/NifA and GlnK was analyzed and biochemical and genetic approaches were carried out to determine electron transfer onto NifL and to examine which amino acid residues are necessary for membrane association.

\section{Complex formation between MBP-NifL/NifA and GInK effecting this complex.}

The regulation of nitrogen fixation occurs in $K$. pneumoniae via direct protein-protein interactions. In the recent work we demonstrated by pull-down experiments that MBP- 
NifL and NifA form inhibitory complexes with a stoichiometric 1:1 ratio independently of the prevalent growth conditions. Under nitrogen fixing conditions $\left(-\mathrm{N} /-\mathrm{O}_{2}\right)$, however, only approximately $1 \%$ of inhibitory complexes were formed as compared to conditions inappropriate for nitrogen fixation which is due to the fact that under the respective conditions the majority of MBP-NifL is sequestered to the cytoplasmic membrane. Sequestration of NifL to the cytoplasmic membrane is the key regulatory mechanism and exclusively responsible for NifA activity and consequently nif-gene expression. Shift experiments from nitrogen fixing conditions $\left(-\mathrm{N} /-\mathrm{O}_{2}\right)$ to either nitrogen or oxygen excess revealed that $85 \%$ to $90 \%$ of MBP-NifL was located in the cytoplasm (Chapter 2). The nitrogen signal is transduced towards the NifL/NifA system via the PII-like protein GlnK. By pull-down experiments it has been demonstrated that GlnK binds to MBP-NifL. Unexpectedly pull-down experiments with MBP-NifA and GlnK showed that GlnK also directly interacts with NifA although interaction appeared to be less intense compared to MBP-NifL. Due to these findings the question arises whether GlnK is even able to bind to the NifL/NifA complex. Thus we carried out pull-down experiments with the three regulatory proteins expressed $\mathrm{NtrC}$ independently and demonstrated for the first time that in K. pneumoniae MBP-NifL/NifA and GlnK form trimeric complexes which are presumably transient during nitrogen signalling. Promoterfusion experiments further revealed that binding of GlnK to the inhibitory NifL/NifA complex yields in the destabilization of the complex allowing NifL to be sequestered to the cytoplasmic membrane under anaerobic conditions (Chapter 2). Complex formation of NifL, NifA and GlnK has been investigated also in $A$. vinelandii and, interestingly, species specific differences have been obtained concerning nitrogen signal transduction in K. pneumoniae and A. vinelandii. In contrary to K. pneumoniae, where modification of GlnK has no direct effect on the function of NifL/NifA complexes, unmodified GlnK stimulates the inhibitory form of NifL under nitrogen excess. Uridylylated GlnK which reflects conditions of nitrogen limitation is not able to activate inhibitory function of NifL. Complex formation of GlnK and the C-terminal GHKL domain of NifL has been demonstrated for unmodified GlnK preventing NifA activity by NifL (Little and Dixon, 2003; Martinez-Argudo et al., 2005). Even in the presence of 2-oxoglutarate, which has been shown to bind to NifA resulting in NifL dissociation from the inhibitory NifA/NifL complexes, unmodified GlnK antagonizes the inducing effect of 2-oxoglutarate and promotes the formation of inhibiting ternary GlnK/NifA/NifL complexes under conditions inappropriate for nitrogen fixation. (Martinez-Argudo et al., 2005). 


\section{Electron transfer onto the FAD-cofactor of NifL.}

NifL reduction in $K$. pneumoniae occurs by electrons derived from the reduced menaquinone pool under anaerobic conditions. To determine whether this electron transfer is achieved directly from menaquinones to the NifL bound FAD-cofactor or whether further oxidoreductases are required for reduction we established an in vitro assay monitoring the reduction of the FAD moiety spectroscopically. (i) A soluble formate dehydrogenase from $W$. succinogenes is able to transfer electrons derived from formate oxidation onto MBP-NifL in the presence of a soluble quinone analogon as electron mediator. (ii) MBP-NifL reduction was observed using liposomes reconstituted with a partial anaerobic respiratory chain containing $\mathrm{Fdh}$ and menaquinones purified from $W$. succinogenes. This clearly proves that electron transfer onto NifL occurs directly from the menaquinone pool and does not require any further $K$. pneumoniae specific oxidoreductase(s) (Chapter 3). As nitrogen fixation is a highly energy consuming process it is attractive to speculate that linking the regulatory mechanism of membrane sequestration to the anaerobic respiratory chain provides the possibility to integrate the energy status of the cell to the regulation of nitrogen fixation. Under anaerobic conditions the fully reduced menaquinone pool might also function as signal for a high energy status whereas a partially reduced menaquinone pool potentially is not able to transfer electrons onto NifL bound FAD. Other mechanism of sensing the energy status via NifL have not been demonstrated for K. pneumoniae yet whereas in A. vinelandii strong evidence has been obtained that the energy status is transduced towards NifL by binding of adenosine nucleotides to the C-terminal GHKL domain of NifL. Dixon and coworkers propose a model for A. vinelandii in which the intracellular ADP/ATP ratio reflects the energy status of the cell. Binding of ADP activates the inhibitory function of NifL resulting in complex formation with NifA whereas high amounts of ATP under conditions appropriate for nitrogen fixation might stimulate NifA and $\sigma^{54}$-RNA-Polymerase association (Money et al., 1999; Money et al., 2001).

\section{NifL membrane association upon reduction occurs unspecifically.}

The key regulatory mechanism of regulating NifA-activity and thus nif-gene expression is the localizational change of NifL to the cytoplasmic membrane upon FAD reduction under nitrogen fixing conditions. As the physiological electron donor for NifL is apparently the 
reduced menaquinone pool located in the cytoplasmic membrane we investigated in vitro whether reduction of FAD itself is sufficient to sequester NifL to the cytoplasmic membrane or whether K. pneumoniae specific receptor proteins are involved in associating the reduced NifL to the membrane. Using the artificial membrane system containing of proteoliposomes reconstituted with Fdh and menaquinones of $W$. succinogenes the FADcofactor of MBP-NifL was reduced. After reduction the samples were loaded on a sucrose density gradient containing formate in excess to ensure reducing conditions throughout the experiment. After ultracentrifugation we demonstrated that reduced MBP-NifL was detected in the same fractions as in which the liposomes were present. On contrary oxidized MBP-NifL was mainly found in fractions with lower sucrose concentrations than the proteoliposomes due to the protein's minor mass compared to the mass of proteoliposomes (Chapter 3). Thus we concluded that membrane association of NifL occurs apparently unspecific and is not dependent on K. pneumoniae specific constituents at all.

A comparable regulatory mechanism is known for PutA (proline utilization protein $\underline{\mathrm{A}}$ ) in Escherichia coli and Salmonella typhimurium. PutA, a flavoprotein consisting of a FADcofactor, is described to be bifunctional. In the presence of high concentrations of proline PutA catalyzes the two-step oxidation of proline to glutamate. The electrons derived from this reaction consequently lead to reduction of the PutA bound FAD-cofactor resulting in membrane association of the protein due to a $>300$ fold increased membrane binding affinity of reduced PutA (Brown and Wood, 1993; Zhang et al., 2004). Additionally membrane association of the reduced PutA leads to dissociation of the PutA-DNA complex, which represses the transcription of put-genes under low concentrations of proline (Maloy, 1987; Muro-Pastor et al., 1997; Ostrovsky de Spicer et al., 1991a; Wood, 1987; Zhang et al., 2004).

However, in the put-regulatory system electrons are not derived from quinones but directly from the reaction catalyzed by the PutA protein. In other regulatory systems where the redox state of quinones is used for transducing red-ox signals to regulate gene expression, it has been demonstrated for a few examples that exclusively the oxidative power of quinones is employed under aerobic conditions. Georgellis and collegues showed e.g. for E. coli that oxidized ubiquinones inhibit autophosphorylation of $\mathrm{ArcB}$, the sensor kinase of the global transcriptional regulatory Arc two-component system, during aerobiosis. Under these conditions the dephosphorylated response regulator ArcA induces the transcription of many genes involved in respiratory metabolism. Under anaerobic conditions the 
phosphorylated ArcA-P activates gene expression of proteins involved in fermentative metabolism (Georgellis et al., 2001; Sawers and Suppmann, 1992). Further it was shown for $\mathrm{ArcB}$ that the oxidative power of ubiquinones generates a disulfide bond in two adjacent cysteine residues resulting in reduced kinase activity of ArcB (Malpica et al., 2004). Another regulatory system using the oxidative power of quinones has been demonstrated for the RegA/RegB two-component system of Rhodobacter capsulatus. RegA is one of the global red-ox regulators which is responsible for the transcriptional control of e.g. compounds of aerobic and anaerobic respiratory chains, compounds of a bacterial photosystem and other cellular processes under red-ox control such as carbon fixation, nitrogen fixation and utilization of hydrogen (Dubbs et al., 2000; Elsen et al., 2000; Madigan and Gest, 1978). Here it was shown that autophosphorylation of the sensor kinase RegB is inhibited by the addition of oxidized coenzyme $\mathrm{Q}_{1}$ whereas the addition of reduced $\mathrm{Q}_{1}$ did not have an inhibitory effect on phosphorylation (Swem et al., 2006). To exclude that ubiquinone does not affect the redox state of amino acid residue $\mathrm{C} 265$, which has been shown to influence autophosphorylation, the addition of oxidized $\mathrm{Q}_{1}$ to a $\mathrm{RegB}$ mutant $(\mathrm{C} 265 \mathrm{~A})$ still results in inhibition of autophosphorylation showing that Q1 overrides the effect of C265. However, it can not be excluded that besides the redox mechanism also an allosteric effect of ubiquinone is involved in regulating kinase activity of RegB (Swem et al., 2006). Due to these findings and the findings obtained for the ArcA/ArcB system Bauer and coworkers propose that the interaction between cysteine residues and ubiquinones might be a widespread mechanism for red-ox sensing and red-ox signalling (Swem et al., 2006).

However the findings obtained by reducing NifL using reconstituted proteoliposomes strongly indicate that in the nif-regulatory system the reducing power of menaquinones is involved in oxygen signalling.

\section{Mutational analysis of NifL to reveal amino acids residues involved in oxygen signal transduction.}

To identify crucial amino acid mutations in the N-terminal domain of NifL responsible for the uptake of the oxygen signal we performed a PCR-based mutagenesis approach to introduce mutations to NifL and established a screening system based on a nif (no growth) under nitrogen fixing conditions. The nif - ${ }^{-}$phenotype results from mutated NifL* proteins no longer able to release inhibition of NifA as they carry mutations at 
amino acid residues which are either involved in contacting the cytoplasmic membrane or which are required to stabilize the FAD-cofactor of NifL. 11.500 mutants carrying an additional mutated nifL* - gene on plasmid pRS315 were screened resulting in 67 clones showing a nif ${ }^{-}$-phenotype. After predicting the localization of the mutated amino acid residues based on the resolved structure of the A. vinelandii PAS1 domain of NifL we identified mutations which were presumably located on the protein surface or which were likely to be situated near the FAD-cofactor (Key et al., 2007). Subsequently the nif phenotype was confirmed for clones carrying only one amino acid mutations: R80C and Q57L. Mutation of R80C presumably is defective in coordinating the FAD-cofactor whereas Q57L probably is located on the surface of NifL yielding in reduced affinity to the cytoplasmic membrane. These findings clearly indicate that single amino acid residues of the N-terminal NifL domain are sufficient to disrupt the transduction of the oxygen signal (Chapter 4). Besides R80, four further amino acid residues have been identified for A. vinelandii NifL which directly coordinate the FAD-cofactor within the protein: N69, E70, W87 and N102 (Key et al., 2007). Except E70 these residues are conserved in K. pneumoniae NifL therefore one might assume that mutation of these residues also lead to mutants conferring a nif incorporated into the protein. Further experiments have to elucidate whether mutations of other residues predicted to be involved in oxygen signalling are also able to disconnect the oxygen signal from nitrogen fixation.

\section{Current working model for oxygen and nitrogen signalling in $K$. pneumoniae.}

Taking together the recent findings concerning nitrogen and oxygen signalling in K. pneumoniae we postulate the following working model for signal transduction onto the nif-regulatory system (see also Fig. 5.1). The nitrogen signal is mediated by the PII-like protein GlnK. Under nitrogen limiting conditions GlnK is NtrC dependently synthesized and forms trimeric complexes in vivo. Each monomer of GlnK is uridylylated in the absence of combined nitrogen, however, the modification of the protein appears not to be essential for transducing the signal of nitrogen limitation onto the NifL/NifA system. Under nitrogen fixing $\left(-\mathrm{N} /-\mathrm{O}_{2}\right)$ conditions modified $\mathrm{GlnK}$ directly interacts with the inhibitory NifL/NifA complex and presumably forms a transient trimeric complex allowing NifL to dissociate. Subsequently NifL is sequestered to the cytoplasmic membrane upon unspecific FAD reduction by electrons derived from the reduced menaquinone pool $\left(\mathrm{MQ}_{\mathrm{red}}\right)$. Under anaerobic conditions oxidoreductases (OR) required for anaerobic 
metabolism are expressed Fnr (fumarate nitrate reductase regulator) dependently. These oxidoreductases transfer the reducing equivalents occurring during anaerobic growth to the menaquinone pool resulting in its reduction (Melville and Gunsalus, 1996; Spiro and Guest, 1990; Spiro, 1994; Unden and Schirawski, 1997).

Results obtained by shift experiments complete the model concerning other environmental conditions. Upon shift to nitrogen excess $\left(+\mathrm{N} /-\mathrm{O}_{2}\right) \mathrm{GlnK}$ is deuridylylated and sequestered to the cytoplasmic membrane either in an AmtB dependent manner comparable to E. coli GlnK (Conroy et al., 2007; Coutts et al., 2002) or potentially in a NifL dependent manner. However, the precise mechanism of GlnK sequestration has to be further investigated. Subsequently under conditions of nitrogen excess further synthesis of NifL, NifA and GlnK is repressed NtrC dependently.

Upon nitrogen limitation and a shift to aerobic growth $\left(-\mathrm{N} /+\mathrm{O}_{2}\right)$ the uridylylated $\mathrm{GlnK}$ interacts with the NifL/NifA complex. Due to rapid oxidation rate of the membrane associated NifL the localization of the protein changes and the majority of NifL is found to be located in the cytoplasm. As the menaquinone pool immediately is oxidized and the transcriptional activator Fnr is inactivated by molecular oxygen (Green et al., 1996; Kiley and Beinert, 1998; Unden and Schirawski, 1997) newly synthesized NifL is not reduced anymore thus not adhering at the cytoplasmic membrane yielding in inhibitory NifL/NifA complexes (indicated in Chapter 3).

In cells growing under nitrogen and oxygen excess $\left(+\mathrm{N} /+\mathrm{O}_{2}\right)$ inhibitory complexes are formed in the cytoplasm and nif-gene expression is inhibited. 

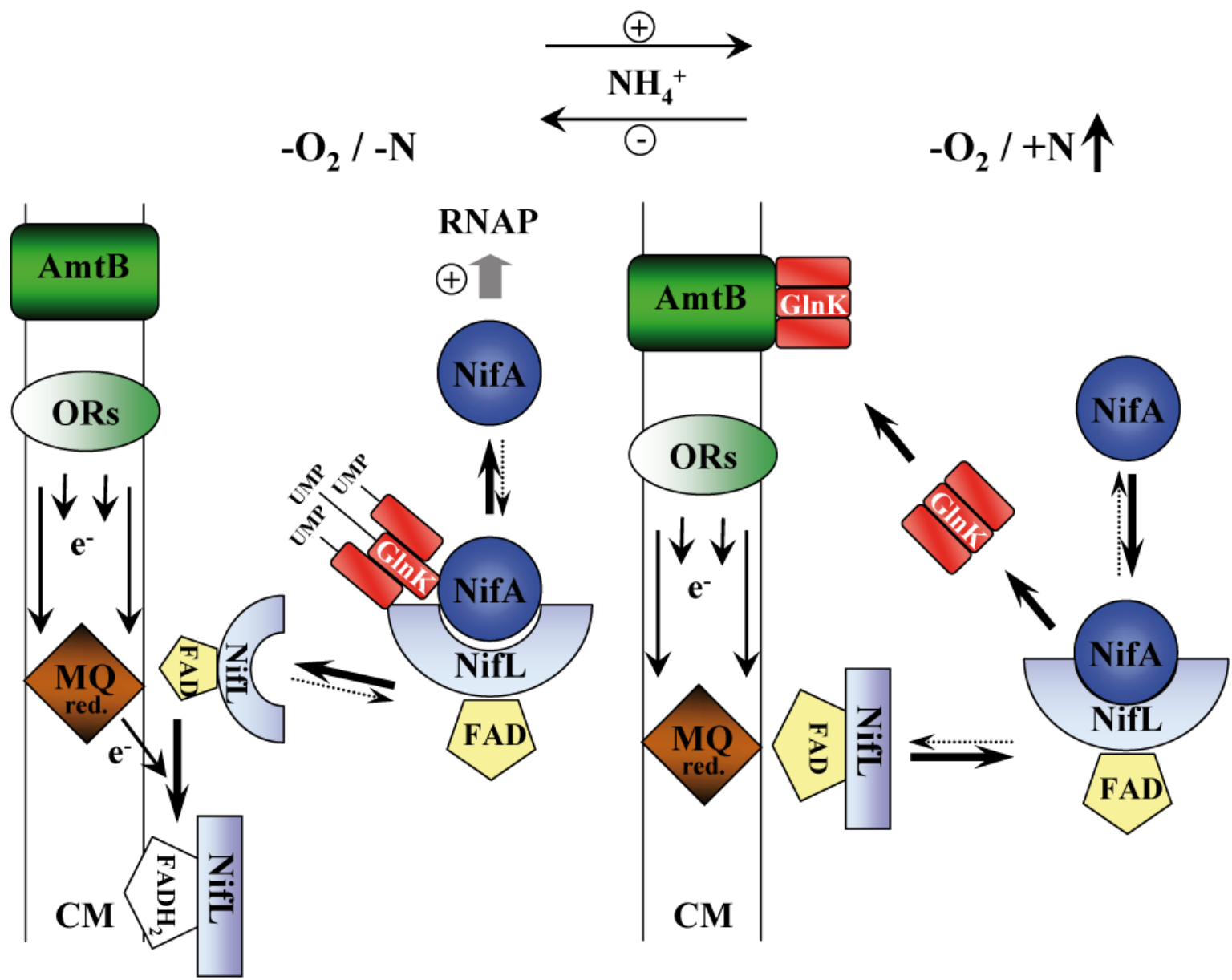

$\oplus \mid \sigma^{N} \odot$

个 $+\mathrm{O}_{2} /-\mathrm{N}$

$\oplus \mid 0^{N} \odot$

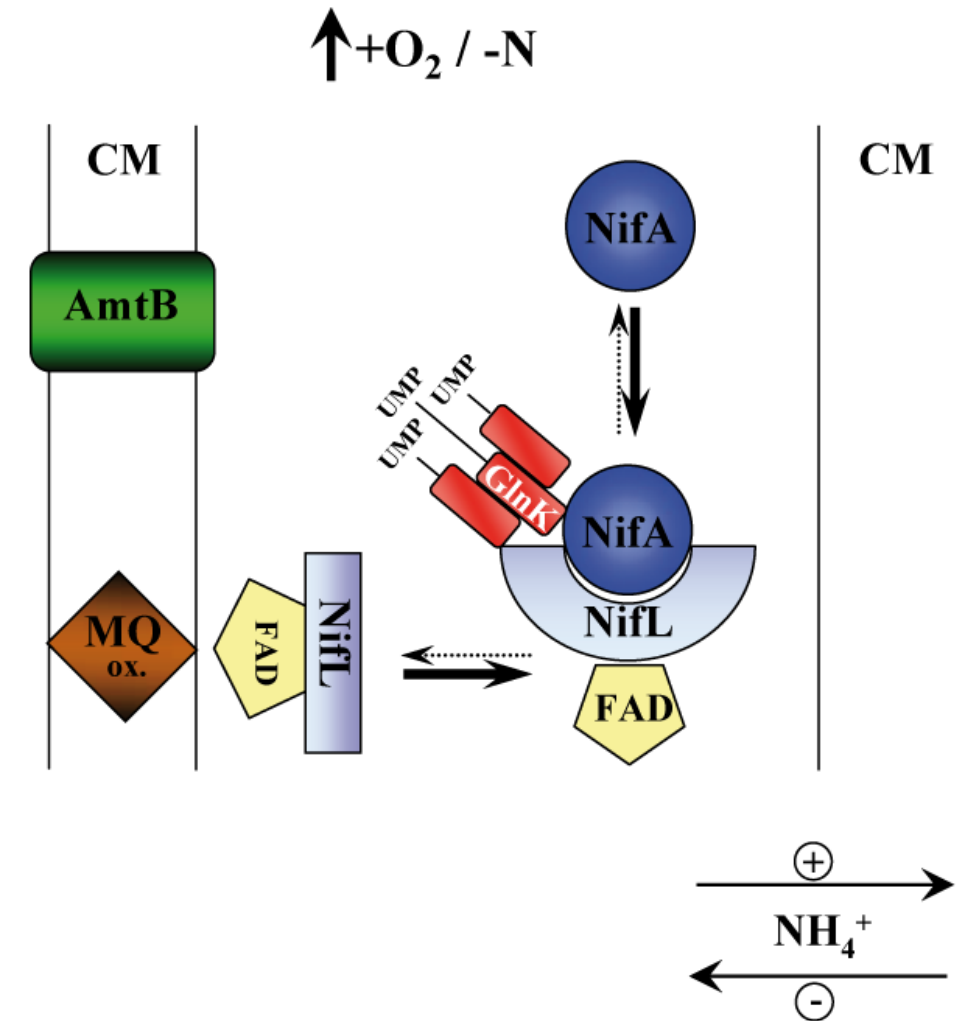

Figure 5.1: Current working model for nitrogen and oxygen signal transduction onto the NifA/NifL regulatory system in $K$. pneumoniae. 


\section{Further studies:}

- Clones carrying a mutagenized nifL* on pRS315 showing a nif-phenotype under nitrogen fixing conditions have to be biochemically analyzed. After separation of single mutated amino acid residues with confirmed nif--phenotype indicating a crucial role in oxygen signalling different experiments have to follow to further characterize NifL.

(i) It is necessary to perform membrane preparations to demonstrate that mutated NifL* is defective in membrane sequestration. These experiments should be performed in an E. coli background lacking chromosomal wild type nifL. Additionally membrane association of NifL* can be verified by sucrose density centrifugation using chemically reduced MBP-NifL* or using soluble Fdh in the presence of formate and a soluble electron mediator for MBP-NifL* reduction incubated with liposomes. Mutated NifL defective in membrane association presumably will not interact with liposomes.

(ii) Furthermore binding characteristics of mutated NifL* could be revealed by Biacore experiments using immobilized proteoliposomes containing Fdh. Analyzing the interaction of reduced mutated NifL* with proteoliposomes preincubated in the presence of excess formate (reducing conditions) should reveal whether single amino acid mutations prevent NifL binding to proteoliposomes.

(iii) Clones carrying mutations predicted to be involved in FAD coordination have to be investigated concerning the relative amount of FAD-cofactor in the protein. Therefore FAD extraction experiments need to be performed to determine if single amino acid mutations result in NifL-apoenzyme.

(iv) Pull-down experiments with "super-inhibiting" C-terminal mutants will potentially reveal which residues of NifL directly interact with NifA or which amino acid mutations yielding in a locked-to-NifA conformation of NifL. To determine which amino acid residues of NifA are involved in binding NifL it would be interesting to develop a screen for NifA suppressor mutants restoring dissociation of "super-inhibiting" NifL/NifA complexes in NifL mutations.

(v) Besides N-terminal and C-terminal domains of NifL, mutations also occurred in the Q-linker region possibly altering the conformation of NifL. However, it remained unclear whether this region is directly involved in transducing the oxygen signal onto NifA in K. pneumoniae. Partial tryptic digestion experiments of NifL* carrying mutations exclusively in the Q-linker region could reveal whether this mutations result in a conformational change which is responsible for nif ${ }^{-}$mutants. 
- Additionally it would be interesting to crystallize the PAS-domain of K. pneumoniae NifL to compare the structure with A. vinelandii NifL and to further investigate FADbinding, electron transfer and the surface structure.

- Interestingly, under oxygen limitation and nitrogen sufficiency $70 \pm 5 \%$ of NtrC independently synthesized GlnK under control of the inducable Tac-promoter expressed from a plasmid appeared to be membrane associated in K. pneumoniae. In E. coli GlnK binds to AmtB thus resulting in membrane association of GlnK under nitrogen limitation. As K. pneumoniae AmtB is not expressed under nitrogen sufficiency GlnK appears to be differently sequestered to the cytoplasmic membrane. To examine in which way sequestration of GlnK occurs in vivo membrane preparations of different mutant strains ( $\Delta$ amtB, $\Delta$ nifL) have to be carried out. 
References:

Arcondeguy, T., van Heeswijk, W.C., and Merrick, M. (1999) Studies on the roles of GlnK and GlnB in regulating Klebsiella pneumoniae NifL-dependent nitrogen control. FEMS Microbiol Lett 180: 263-270.

Arcondeguy, T., Lawson, D., and Merrick, M. (2000) Two residues in the T-loop of GlnK determine NifL-dependent nitrogen control of nif gene expression. J Biol Chem 275: 38452-38456.

Arcondeguy, T., Jack, R., and Merrick, M. (2001) P(II) signal transduction proteins, pivotal players in microbial nitrogen control. Microbiol Mol Biol Rev 65: 80-105.

Arnott, M., Sidoti, C., Hill, S., and Merrick, M. (1989) Deletion analysis of the nitrogen fixation regulatory gene nifL of Klebsiella pneumoniae. Arch. Microbiol. 151: $180-182$.

Arsene, F., Kaminski, P.A., and Elmerich, C. (1996) Modulation of NifA activity by PII in Azospirillum brasilense: evidence for a regulatory role of the NifA N-terminal domain. J Bacteriol 178: 4830-4838.

Arsene, F., Kaminski, P.A., and Elmerich, C. (1999) Control of Azospirillum brasilense NifA activity by P(II): effect of replacing Tyr residues of the NifA Nterminal domain on NifA activity. FEMS Microbiol Lett 179: 339-343.

Atkinson, M.R., and Ninfa, A.J. (1999) Characterization of the GlnK protein of Escherichia coli. Mol Microbiol 32: 301-313.

Austin, S., Buck, M., Cannon, W., Eydmann, T., and Dixon, R. (1994) Purification and in vitro activities of the native nitrogen fixation control proteins NifA and NifL. J Bacteriol 176: 3460-3465.

Belay, N., Sparling, R., and Daniels, L. (1984) Dinitrogen fixation by a thermophilic methanogenic bacterium. Nature 312: 286-288.

Berger, D.K., Narberhaus, F., and Kustu, S. (1994) The isolated catalytic domain of NIFA, a bacterial enhancer-binding protein, activates transcription in vitro: activation is inhibited by NIFL. Proc Natl Acad Sci U S A 91: 103-107.

Berger, D.K., Narberhaus, F., Lee, H.S., and Kustu, S. (1995) In vitro studies of the domains of the nitrogen fixation regulatory protein NIFA. J Bacteriol 177: 191-199.

Bergmayer, H. (1983) Methods of enzymatic analysis. In Methods of enzymatic analysis. Vol. II. Weinheim: Verlag Chemie. 
Biel, S., Simon, J., Gross, R., Ruiz, T., Ruitenberg, M., and Kroger, A. (2002) Reconstitution of coupled fumarate respiration in liposomes by incorporating the electron transport enzymes isolated from Wolinella succinogenes. Eur J Biochem 269: 1974-1983.

Blanco, G., Drummond, M., Woodley, P., and Kennedy, C. (1993) Sequence and molecular analysis of the nifL gene of Azotobacter vinelandii. Mol Microbiol 9: 869-879.

Bock, A., and Gross, R. (2002) The unorthodox histidine kinases BvgS and EvgS are responsive to the oxidation status of a quinone electron carrier. Eur J Biochem 269: 3479-3484.

Brown, E.D., and Wood, J.M. (1993) Conformational change and membrane association of the PutA protein are coincident with reduction of its FAD-cofactor by proline. J Biol Chem 268: 8972-8979.

Buck, M., Cannon, W., and Woodcock, J. (1987) Transcriptional activation of the Klebsiella pneumoniae nitrogenase promoter may involve DNA loop formation. Mol Microbiol 1: 243-249.

Burgess, B.K., and Lowe, D.J. (1996) Mechanism of Molybdenum Nitrogenase. Chem Rev 96: 2983-3012.

Conroy, M.J., Durand, A., Lupo, D., Li, X.D., Bullough, P.A., Winkler, F.K., and Merrick, M. (2007) The crystal structure of the Escherichia coli AmtB-GlnK complex reveals how GlnK regulates the ammonia channel. Proc Natl Acad Sci U S A 104: 1213-1218.

Coutts, G., Thomas, G., Blakey, D., and Merrick, M. (2002) Membrane sequestration of the signal transduction protein GlnK by the ammonium transporter AmtB. Embo $J$ 21: $536-545$.

Daesch, G., and Mortenson, L.E. (1972) Effect of ammonia on the synthesis and function of the $\mathrm{N} 2$-fixing enzyme system in Clostridium pasteurianum. J Bacteriol 110: 103-109.

de Lorenzo, V., Herrero, M., Jakubzik, U., and Timmis, K.N. (1990) Mini-Tn5 transposon derivatives for insertion mutagenesis, promoter probing, and chromosomal insertion of cloned DNA in gram-negative eubacteria. $J$ Bacteriol 172: 6568-6572. 
Dean, D.R., and Jacobson, M.R. (1992) Biochemical genetics of nitrogenase. In Biological nitrogen fixation. Stacey, G., Burris, R.H. and Evans, H.J. (eds). New York: Chapman \& Hall, pp. 763-834.

Deppenmeier, U., Johann, A., Hartsch, T., Merkl, R., Schmitz, R.A., MartinezArias, R., Henne, A., Wiezer, A., Baumer, S., Jacobi, C., Bruggemann, H., Lienard, T., Christmann, A., Bomeke, M., Steckel, S., Bhattacharyya, A., Lykidis, A., Overbeek, R., Klenk, H.P., Gunsalus, R.P., Fritz, H.J., and Gottschalk, G. (2002) The genome of Methanosarcina mazei: evidence for lateral gene transfer between bacteria and archaea. J Mol Microbiol Biotechnol 4: 453-461.

Dietrich, W., and Klimmek, O. (2002) The function of methyl-menaquinone-6 and polysulfide reductase membrane anchor (PsrC) in polysulfide respiration of Wolinella succinogenes. Eur J Biochem 269: 1086-1095.

Dixon, R. (1998) The oxygen-responsive NIFL-NIFA complex: a novel two-component regulatory system controlling nitrogenase synthesis in gamma-proteobacteria. Arch Microbiol 169: 371-380.

Drepper, T., Gross, S., Yakunin, A.F., Hallenbeck, P.C., Masepohl, B., and Klipp, W. (2003) Role of GlnB and GlnK in ammonium control of both nitrogenase systems in the phototrophic bacterium Rhodobacter capsulatus. Microbiology 149: 2203-2212.

Dross, F., Geisler, V., Lenger, R., Theis, F., Krafft, T., Fahrenholz, F., Kojro, E., Duchene, A., Tripier, D., Juvenal, K., and et al. (1992) The quinone-reactive Ni/Fe-hydrogenase of Wolinella succinogenes. Eur J Biochem 206: 93-102.

Drummond, M.H., and Wootton, J.C. (1987) Sequence of nifL from Klebsiella pneumoniae: mode of action and relationship to two families of regulatory proteins. Mol Microbiol 1: 37-44.

Drummond, M.H., Contreras, A., and Mitchenall, L.A. (1990) The function of isolated domains and chimaeric proteins constructed from the transcriptional activators NifA and NtrC of Klebsiella pneumoniae. Mol Microbiol 4: 29-37.

Dubbs, J.M., Bird, T.H., Bauer, C.E., and Tabita, F.R. (2000) Interaction of CbbR and RegA* transcription regulators with the Rhodobacter sphaeroides cbbIPromoteroperator region. J Biol Chem 275: 19224-19230.

Egener, T., Sarkar, A., Martin, D.E., and Reinhold-Hurek, B. (2002) Identification of a NifL-like protein in a diazotroph of the beta-subgroup of the Proteobacteria, Azoarcus sp. strain BH72. Microbiology 148: 3203-3212. 
Ehlers, C., Grabbe, R., Veit, K., and Schmitz, R.A. (2002) Characterization of GlnK1 from Methanosarcina mazei strain Go1: complementation of an Escherichia coli glnK mutant strain by GlnK1. J Bacteriol 184: 1028-1040.

Elsen, S., Dischert, W., Colbeau, A., and Bauer, C.E. (2000) Expression of uptake hydrogenase and molybdenum nitrogenase in Rhodobacter capsulatus is coregulated by the RegB-RegA two-component regulatory system. J Bacteriol 182: 2831-2837.

Filser, M., Merrick, M., and Cannon, F. (1983) Cloning and characterisation of nifLA regulatory mutations from Klebsiella pneumoniae. Mol Gen Genet 191: 485-491.

Fischer, H.M. (1994) Genetic regulation of nitrogen fixation in rhizobia. Microbiol Rev 58: $352-386$.

Fischer, H.M. (1996) Environmental regulation of rhizobial symbiotic nitrogen fixation genes. Trends Microbiol 4: 317-320.

Fluckiger, R., Paz, M.A., and Gallop, P.M. (1995) Redox-cycling detection of dialyzable pyrroloquinoline quinone and quinoproteins. Methods Enzymol 258: 140149.

Forchhammer, K., and Hedler, A. (1997) Phosphoprotein PII from cyanobacteria-analysis of functional conservation with the PII signal-transduction protein from Escherichia coli. Eur J Biochem 244: 869-875.

Galagan, J.E., Nusbaum, C., Roy, A., Endrizzi, M.G., Macdonald, P., FitzHugh, W., Calvo, S., Engels, R., Smirnov, S., Atnoor, D., Brown, A., Allen, N., Naylor, J., Stange-Thomann, N., DeArellano, K., Johnson, R., Linton, L., McEwan, P., McKernan, K., Talamas, J., Tirrell, A., Ye, W., Zimmer, A., Barber, R.D., Cann, I., Graham, D.E., Grahame, D.A., Guss, A.M., Hedderich, R., IngramSmith, C., Kuettner, H.C., Krzycki, J.A., Leigh, J.A., Li, W., Liu, J., Mukhopadhyay, B., Reeve, J.N., Smith, K., Springer, T.A., Umayam, L.A., White, O., White, R.H., Conway de Macario, E., Ferry, J.G., Jarrell, K.F., Jing, H., Macario, A.J., Paulsen, I., Pritchett, M., Sowers, K.R., Swanson, R.V., Zinder, S.H., Lander, E., Metcalf, W.W., and Birren, B. (2002) The genome of M. acetivorans reveals extensive metabolic and physiological diversity. Genome Res 12: $532-542$.

Georgellis, D., Kwon, O., and Lin, E.C. (2001) Quinones as the redox signal for the arc two-component system of bacteria. Science 292: 2314-2316.

Govantes, F., Andujar, E., and Santero, E. (1998) Mechanism of translational coupling in the nifLA operon of Klebsiella pneumoniae. Embo J 17: 2368-2377. 
Grabbe, R., Klopprogge, K., and Schmitz, R.A. (2001) Fnr Is required for NifLdependent oxygen control of nif gene expression in Klebsiella pneumoniae. $J$ Bacteriol 183: 1385-1393.

Grabbe, R., and Schmitz, R.A. (2003) Oxygen control of nif gene expression in Klebsiella pneumoniae depends on NifL reduction at the cytoplasmic membrane by electrons derived from the reduced quinone pool. Eur J Biochem 270: 1555-1566.

Green, J., Bennett, B., Jordan, P., Ralph, E.T., Thomson, A.J., and Guest, J.R. (1996) Reconstitution of the [4Fe-4S] cluster in FNR and demonstration of the aerobic-anaerobic transcription switch in vitro. Biochem J 316 ( Pt 3): 887-892.

Gross, R., Pisa, R., Sanger, M., Lancaster, C.R., and Simon, J. (2004) Characterization of the menaquinone reduction site in the diheme cytochrome $b$ membrane anchor of Wolinella succinogenes NiFe-hydrogenase. J Biol Chem 279: 274-281.

Halbleib, C.M., and Ludden, P.W. (2000) Regulation of biological nitrogen fixation. $J$ Nutr 130: 1081-1084.

He, L., Soupene, E., Ninfa, A., and Kustu, S. (1998) Physiological role for the GlnK protein of enteric bacteria: relief of NifL inhibition under nitrogen-limiting conditions. J Bacteriol 180: 6661-6667.

Henderson, N., Austin, S., and Dixon, R. (1989) Role of metal ions in negative regulation of nitrogen fixation by the nifL gene product from Klebsiella pneumoniae. Mol. Gen. Genet. 216: 484-491.

Hill, S., Kennedy, C., Kavanagh, E., Goldberg, R.B., and Hanau, R. (1981) Nitrogen fixation gene (nifL) involved in oxygen regulation of nitrogenase synthesis in $\mathrm{K}$. pneumoniae. Nature 290: 424-426.

Hill, S., Austin, S., Eydmann, T., Jones, T., and Dixon, R. (1996) Azotobacter vinelandii NIFL is a flavoprotein that modulates transcriptional activation of nitrogen-fixation genes via a redox-sensitive switch. Proc Natl Acad Sci U S A 93: 2143-2148.

Hoover, T.R., Santero, E., Porter, S., and Kustu, S. (1990) The integration host factor stimulates interaction of RNA polymerase with NIFA, the transcriptional activator for nitrogen fixation operons. Cell 63: 11-22.

Howard, J.B., and Rees, D.C. (1996) Structural Basis of Biological Nitrogen Fixation. Chem Rev 96: 2965-2982. 
Inoue, H., Nojima, H., and Okayama, H. (1990) High efficiency transformation of Escherichia coli with plasmids. Gene 96: 23-28.

Iverson, T.M., Luna-Chavez, C., Cecchini, G., and Rees, D.C. (1999) Structure of the Escherichia coli fumarate reductase respiratory complex. Science 284: 1961-1966.

Jack, R., De Zamaroczy, M., and Merrick, M. (1999) The signal transduction protein GlnK is required for NifL-dependent nitrogen control of nif gene expression in Klebsiella pneumoniae. J Bacteriol 181: 1156-1162.

Kessler, P.S., McLarnan, J., and Leigh, J.A. (1997) Nitrogenase phylogeny and the molybdenum dependence of nitrogen fixation in Methanococcus maripaludis. $J$ Bacteriol 179: 541-543.

Kessler, P.S., Blank, C., and Leigh, J.A. (1998) The nif gene operon of the methanogenic archaeon Methanococcus maripaludis. J Bacteriol 180: 1504-1511.

Key, J., Hefti, M., Purcell, E.B., and Moffat, K. (2007) Structure of the redox sensor domain of Azotobacter vinelandii NifL at atomic resolution: signaling, dimerization, and mechanism. Biochemistry 46: 3614-3623.

Kiley, P.J., and Beinert, H. (1998) Oxygen sensing by the global regulator, FNR: the role of the iron-sulfur cluster. FEMS Microbiol Rev 22: 341-352.

Klopprogge, K., and Schmitz, R.A. (1999) NifL of Klebsiella pneumoniae: redox characterization in relation to the nitrogen source. Biochim Biophys Acta 1431: $462-$ 470 .

Klopprogge, K., Grabbe, R., Hoppert, M., and Schmitz, R.A. (2002) Membrane association of Klebsiella pneumoniae NifL is affected by molecular oxygen and combined nitrogen. Arch Microbiol 177: 223-234.

Kröger, A., V. Geisler and A. Duchêne (1994) Isolation of Wolinella succinegenes hydrogenase, Chromatofucusing. In A practical guide to membrane protein purification. von Jagow, G. and Schägger, H. (eds): Academic Press, pp. 141-147.

Lambert, O., Levy, D., Ranck, J.L., Leblanc, G., and Rigaud, J.L. (1998) A new "gel-like" phase in dodecyl maltoside-lipid mixtures: implications in solubilization and reconstitution studies. Biophys J 74: 918-930.

Lee, H.S., Berger, D.K., and Kustu, S. (1993a) Activity of purified NIFA, a transcriptional activator of nitrogen fixation genes. Proc Natl Acad Sci U S A 90: 2266-2270.

Lee, H.S., Narberhaus, F., and Kustu, S. (1993b) In vitro activity of NifL, a signal transduction protein for biological nitrogen fixation. J Bacteriol 175: 7683-7688. 
Lei, S., Pulakat, L., and Gavini, N. (1999) Genetic analysis of nif regulatory genes by utilizing the yeast two-hybrid system detected formation of a NifL-NifA complex that is implicated in regulated expression of nif genes. J Bacteriol 181: 6535-6539.

Leigh, J.A. (2000) Nitrogen fixation in methanogens: the archaeal perspective. Curr Issues Mol Biol 2: 125-131.

Leung, D.W., Chen, E., and Goeddel, D.V. (1989) A method for random mutagenesis of a defined DNA segment using a modified polymerase chain reaction. Technique A Journal of Methods in Cell and Molecular Biology 1: 11-15.

Liang, Y.Y., de Zamaroczy, M., Arsene, F., Paquelin, A., and Elmerich, C. (1992) Regulation of nitrogen fixation in Azospirillum brasilense Sp7: involvement of nifA, glnA and glnB gene products. FEMS Microbiol Lett 79: 113-119.

Little, R., Reyes-Ramirez, F., Zhang, Y., van Heeswijk, W.C., and Dixon, R. (2000) Signal transduction to the Azotobacter vinelandii NIFL-NIFA regulatory system is influenced directly by interaction with 2-oxoglutarate and the PII regulatory protein. Embo J 19: 6041-6050.

Little, R., Colombo, V., Leech, A., and Dixon, R. (2002) Direct interaction of the NifL regulatory protein with the GlnK signal transducer enables the Azotobacter vinelandii NifL-NifA regulatory system to respond to conditions replete for nitrogen. J Biol Chem 277: 15472-15481.

Little, R., and Dixon, R. (2003) The amino-terminal GAF domain of Azotobacter vinelandii NifA binds 2-oxoglutarate to resist inhibition by NifL under nitrogenlimiting conditions. J Biol Chem 278: 28711-28718.

Little, R., Martinez-Argudo, I., and Dixon, R. (2006) Role of the central region of NifL in conformational switches that regulate nitrogen fixation. Biochem Soc Trans 34: 162-164.

Little, R., Martinez-Argudo, I., Perry, S., and Dixon, R. (2007) Role of the H Domain of the Histidine Kinase-like Protein NifL in Signal Transmission. J Biol Chem 282: 13429-13437.

Lobo, A.L., and Zinder, S.H. (1992) Nitrogen fixation by methanogenic bacteria. In Biological nitrogen fixation. Stacey, G., Burris, R.H. and Evans, H.J. (eds). New York: Chapman \& Hall, pp. 736-762.

Macheroux, P., Hill, S., Austin, S., Eydmann, T., Jones, T., Kim, S.O., Poole, R., and Dixon, R. (1998) Electron donation to the flavoprotein NifL, a redox-sensing transcriptional regulator. Biochem J 332 ( Pt 2): 413-419. 
MacNeil, D., Zhu, J., and Brill, W.J. (1981) Regulation of nitrogen fixation in Klebsiella pneumoniae: isolation and characterization of strains with nif-lac fusions. J Bacteriol 145: 348-357.

Madigan, M.T., and Gest, H. (1978) Growth of a photosynthetic bacterium anaerobically in darkness, supported by "oxidant-dependent" sugar fermentation. Arch Microbiol 117: 119-122.

Maloy, S. (1987) The proline utilization operon. In Salmonella typhimurium: cellular and molecular biology. Neidhardt FC, I.J., Low KB, Magasanik B, Schaechter M, Umbarger HE (ed): American Society for Microbiology, Washington, DC, pp, pp. $1513-1519$.

Malpica, R., Franco, B., Rodriguez, C., Kwon, O., and Georgellis, D. (2004) Identification of a quinone-sensitive redox switch in the ArcB sensor kinase. Proc Natl Acad Sci U S A 101: 13318-13323.

Martinez-Argudo, I., Little, R., and Dixon, R. (2004a) A crucial arginine residue is required for a conformational switch in NifL to regulate nitrogen fixation in Azotobacter vinelandii. Proc Natl Acad Sci U S A 101: 16316-16321.

Martinez-Argudo, I., Little, R., and Dixon, R. (2004b) Role of the amino-terminal GAF domain of the NifA activator in controlling the response to the antiactivator protein NifL. Mol Microbiol 52: 1731-1744.

Martinez-Argudo, I., Little, R., Shearer, N., Johnson, P., and Dixon, R. (2004c) The NifL-NifA System: a multidomain transcriptional regulatory complex that integrates environmental signals. J Bacteriol 186: 601-610.

Martinez-Argudo, I., Little, R., Shearer, N., Johnson, P., and Dixon, R. (2005) Nitrogen fixation: key genetic regulatory mechanisms. Biochem Soc Trans 33: 152156.

Matsson, M., Tolstoy, D., Aasa, R., and Hederstedt, L. (2000) The distal heme center in Bacillus subtilis succinate:quinone reductase is crucial for electron transfer to menaquinone. Biochemistry 39: 8617-8624.

Meletzus, D., Rudnick, P., Doetsch, N., Green, A., and Kennedy, C. (1998) Characterization of the glnK-amtB operon of Azotobacter vinelandii. J Bacteriol 180: $3260-3264$.

Melville, S.B., and Gunsalus, R.P. (1996) Isolation of an oxygen-sensitive FNR protein of Escherichia coli: interaction at activator and repressor sites of FNRcontrolled genes. Proc Natl Acad Sci U S A 93: 1226-1231. 
Merrick, M., S. Hill, H. Hennecke, M. Hahn, R.Dixon, and C. Kennedy (1982) Repressor properties of the nifL gene product in Klebsiella pneumoniae. Mol. Gen. Genet. 185: 75-81.

Merrick, M.J., and Edwards, R.A. (1995) Nitrogen control in bacteria. Microbiol Rev 59: 604-622.

Miller, V.L., and Mekalanos, J.J. (1988) A novel suicide vector and its use in construction of insertion mutations: osmoregulation of outer membrane proteins and virulence determinants in Vibrio cholerae requires toxR. J Bacteriol 170: 25752583.

Money, T., Jones, T., Dixon, R., and Austin, S. (1999) Isolation and properties of the complex between the enhancer binding protein NIFA and the sensor NIFL. $J$ Bacteriol 181: 4461-4468.

Money, T., Barrett, J., Dixon, R., and Austin, S. (2001) Protein-protein interactions in the complex between the enhancer binding protein NIFA and the sensor NIFL from Azotobacter vinelandii. J Bacteriol 183: 1359-1368.

Monteiro, R.A., Souza, E.M., Funayama, S., Yates, M.G., Pedrosa, F.O., and Chubatsu, L.S. (1999a) Expression and functional analysis of an N-truncated NifA protein of Herbaspirillum seropedicae. FEBS Lett 447: 283-286.

Monteiro, R.A., Souza, E.M., Yates, M.G., Pedrosa, F.O., and Chubatsu, L.S. (1999b) In-trans regulation of the N-truncated-NIFA protein of Herbaspirillum seropedicae by the N-terminal domain. FEMS Microbiol Lett 180: 157-161.

Morett, E., and Buck, M. (1989) In vivo studies on the interaction of RNA polymerase-sigma 54 with the Klebsiella pneumoniae and Rhizobium meliloti nifH promoters. J Mol Biol 210: 65-77.

Morett, E., and Segovia, L. (1993) The sigma 54 bacterial enhancer-binding protein family: mechanism of action and phylogenetic relationship of their functional domains. J Bacteriol 175: 6067-6074.

Muro-Pastor, A.M., Ostrovsky, P., and Maloy, S. (1997) Regulation of gene expression by repressor localization: biochemical evidence that membrane and DNA binding by the PutA protein are mutually exclusive. J Bacteriol 179: 2788-2791.

Narberhaus, F., Lee, H.S., Schmitz, R.A., He, L., and Kustu, S. (1995) The Cterminal domain of NifL is sufficient to inhibit NifA activity. $J$ Bacteriol 177: 50785087. 
Ostrovsky de Spicer, P., O'Brien, K., and Maloy, S. (1991a) Regulation of proline utilization in Salmonella typhimurium: a membrane-associated dehydrogenase binds DNA in vitro. $J$ Bacteriol 173: 211-219.

Ostrovsky de Spicer, P., O'Brien, K., and Maloy, S. (1991b) Regulation of proline utilization in Salmonella typhimurium: a membrane-associated dehydrogenase binds DNA in vitro. $J$ Bacteriol 173: 211-219.

Ostrovsky de Spicer, P., and Maloy, S. (1993) PutA protein, a membrane-associated flavin dehydrogenase, acts as a redox-dependent transcriptional regulator. Proc Natl Acad Sci U S A 90: 4295-4298.

Ow, D.W., and Ausubel, F.M. (1983) Regulation of nitrogen metabolism genes by nifA gene product in Klebsiella pneumoniae. Nature 301: 307-313.

Perry, S., Shearer, N., Little, R., and Dixon, R. (2005) Mutational analysis of the nucleotide-binding domain of the anti-activator NifL. J Mol Biol 346: 935-949.

Ray, P., Smith, K.J., Parslow, R.A., Dixon, R., and Hyde, E.I. (2002) Secondary structure and DNA binding by the C-terminal domain of the transcriptional activator NifA from Klebsiella pneumoniae. Nucleic Acids Res 30: 3972-3980.

Rees, D.C., and Howard, J.B. (1999) Structural bioenergetics and energy transduction mechanisms. J Mol Biol 293: 343-350.

Reyes-Ramirez, F., Little, R., Hill, S., Heeswijk, W.v., and Dixon, R. (2000)

Regulation of Azotobacter vinelandii NifA activity by NifL: role of PII-like proteins in nitrogen sensing. In Nitrogen fixation; from molecules to crop productivity. Pedrosa, F.O., Hungria, M., Yates, M.G. and Newton, W.E. (eds). Dordrecht: Kluwer Academic, pp. 97-98.

Reyes-Ramirez, F., Little, R., and Dixon, R. (2001) Role of Escherichia coli nitrogen regulatory genes in the nitrogen response of the Azotobacter vinelandii NifL-NifA complex. J Bacteriol 183: 3076-3082.

Reyes-Ramirez, F., Little, R., and Dixon, R. (2002) Mutant forms of the Azotobacter vinelandii transcriptional activator NifA resistant to inhibition by the NifL regulatory protein. J Bacteriol 184: 6777-6785.

Rudnick, P., Kunz, C., Gunatilaka, M.K., Hines, E.R., and Kennedy, C. (2002) Role of GlnK in NifL-mediated regulation of NifA activity in Azotobacter vinelandii. $J$ Bacteriol 184: 812-820.

Sambrook, J., E.F. Fritsch, and T. Maniatis (1989) Molecular Cloning: A Laboratory Manual. New York: Cold Spring Harbor Laboratory Press. 
Sawers, G., and Suppmann, B. (1992) Anaerobic induction of pyruvate formate-lyase gene expression is mediated by the ArcA and FNR proteins. J Bacteriol 174: 34743478.

Schmitz, R.A., He, L., and Kustu, S. (1996) Iron is required to relieve inhibitory effects on NifL on transcriptional activation by NifA in Klebsiella pneumoniae. $J$ Bacteriol 178: 4679-4687.

Schmitz, R.A. (1997) NifL of Klebsiella pneumoniae carries an N-terminally bound FAD-cofactor, which is not directly required for the inhibitory function of NifL. FEMS Microbiol Lett 157: 313-318.

Schmitz, R.A. (2000) Internal glutamine and glutamate pools in Klebsiella pneumoniae grown under different conditions of nitrogen availability. Curr Microbiol 41: 357362.

Schmitz, R.A., Klopprogge, K., and Grabbe, R. (2002) Regulation of nitrogen fixation in Klebsiella pneumoniae and Azotobacter vinelandii: NifL, transducing two environmental signals to the nif transcriptional activator NifA. J Mol Microbiol Biotechnol 4: 235-242.

Singleton, W.S., Gray, M.S., Brown, M.L., and White, J.L. (1965)

Chromatographically Homogeneous Lecithin from Egg Phospholipids. J Am Oil Chem Soc 42: 53-56.

Soderback, E., Reyes-Ramirez, F., Eydmann, T., Austin, S., Hill, S., and Dixon, R. (1998) The redox- and fixed nitrogen-responsive regulatory protein NIFL from Azotobacter vinelandii comprises discrete flavin and nucleotide-binding domains. Mol Microbiol 28: 179-192.

Spiro, S., and Guest, J.R. (1990) FNR and its role in oxygen-regulated gene expression in Escherichia coli. FEMS Microbiol Rev 6: 399-428.

Spiro, S. (1994) The FNR family of transcriptional regulators. Antonie Van Leeuwenhoek 66: 23-36.

Steenhoudt, O., and Vanderleyden, J. (2000) Azospirillum, a free-living nitrogenfixing bacterium closely associated with grasses: genetic, biochemical and ecological aspects. FEMS Microbiol Rev 24: 487-506.

Stips, J., Thummer, R., Neumann, M., and Schmitz, R.A. (2004) GlnK effects complex formation between NifA and NifL in Klebsiella pneumoniae. Eur J Biochem 271: 3379-3388. 
Swem, L.R., Gong, X., Yu, C.A., and Bauer, C.E. (2006) Identification of a ubiquinone-binding site that affects autophosphorylation of the sensor kinase RegB. J Biol Chem 281: 6768-6775.

Taylor, B.L., and Zhulin, I.B. (1999) PAS domains: internal sensors of oxygen, redox potential, and light. Microbiol Mol Biol Rev 63: 479-506.

Thummer, R., Klimmek, O., and Schmitz, R.A. (2007) Biochemical Studies of Klebsiella pneumoniae NifL Reduction Using Reconstituted Partial Anaerobic Respiratory Chains of Wolinella succinogenes. J Biol Chem 282: 12517-12526.

Unden, G., and Kröger, A. (1982) Reconstitution in liposomes of the electron transport chain catalyzing fumarate reduction by formate. Biochim. Biophys. Acta 682: 258263.

Unden, G., and Kroger, A. (1986) Reconstitution of a functional electron-transfer chain from purified formate dehydrogenase and fumarate reductase complexes. Methods Enzymol 126: 387-399.

Unden, G. (1988) Differential roles for menaquinone and demethylmenaquinone in anaerobic electron transport of E. coli and their fnr-independent expression. Arch Microbiol 150: 499-503.

Unden, G., and Schirawski, J. (1997) The oxygen-responsive transcriptional regulator FNR of Escherichia coli: the search for signals and reactions. Mol Microbiol 25: 205-210.

Upchurch, R.G., and Mortenson, L.E. (1980) In vivo energetics and control of nitrogen fixation: changes in the adenylate energy charge and adenosine 5'diphosphate/adenosine 5'-triphosphate ratio of cells during growth on dinitrogen versus growth on ammonia. J Bacteriol 143: 274-284.

van Heeswijk, W.C., Hoving, S., Molenaar, D., Stegeman, B., Kahn, D., and Westerhoff, H.V. (1996) An alternative PII protein in the regulation of glutamine synthetase in Escherichia coli. Mol Microbiol 21: 133-146.

Wood, J.M. (1987) Membrane association of proline dehydrogenase in Escherichia coli is redox dependent. Proc Natl Acad Sci U S A 84: 373-377.

Xu, Y., Cheah, E., Carr, P.D., van Heeswijk, W.C., Westerhoff, H.V., Vasudevan, S.G., and Ollis, D.L. (1998) GlnK, a PII-homologue: structure reveals ATP binding site and indicates how the T-loops may be involved in molecular recognition. $J$ Mol Biol 282: 149-165. 
Young, J.P.W. (1992) Phylogenetic classification of nitrogen fixing organisms. In Biological nitrogen fixation. Stacey, G., Burris, R.H. and Evans, H.J. (eds). New York: Chapman \& Hall, pp. 43-86.

Zhang, W., Zhou, Y., and Becker, D.F. (2004) Regulation of PutA-membrane associations by flavin adenine dinucleotide reduction. Biochemistry 43: 13165 13174.

Zhang, W., Zhang, M., Zhu, W., Zhou, Y., Wanduragala, S., Rewinkel, D., Tanner, J.J., and Becker, D.F. (2007) Redox-Induced Changes in Flavin Structure and Roles of Flavin N(5) and the Ribityl 2'-OH Group in Regulating PutA-Membrane Binding(,). Biochemistry 46: 483-491.

Zhulin, I.B., Taylor, B.L., and Dixon, R. (1997) PAS domain S-boxes in Archaea, Bacteria and sensors for oxygen and redox. Trends Biochem Sci 22: 331-333. 


\section{Curriculum vitae:}

\section{Robert Thummer}

Date of birth:

15.06.1980

Place of birth:

Alfeld/Leine, Germany

Citizenship:

Austrian

\section{Education:}

$1986-1990$
$1990-1998$

Scientific Background:

Oct. 1998 - Jun. 2003

Sep. 2002 - Jun. 2003

Aug. 2003 - May 2005

since May 2005

since Aug. 2003
Primary Education in Föhrste

Secondary Education in Salzburg (Austria)
Study of Biology at the Georg-August-University, Göttingen

Diploma thesis in Micobiology; Thesis title: "Charakterisierung des NifL-Proteins in Bezug auf die Sauerstoff- und Stickstoffsignaltransduktion in Klebsiella pneumoniae“ (Prof. Dr. G. Gottschalk).

Scientific assistant at the Institute of Microbiology and Genetics, Georg-August-University, Göttingen

Scientific assistant at the Institute of general Microbiology, Christian-Albrecht-University, Göttingen Ph.D. thesis in Microbiology; Thesis title: "Regulation of nitrogen fixation in Klebsiella pneumoniae: Nitrogen and oxygen signal perception by the negative regulator NifL" (Prof. Dr. R. A. Schmitz-Streit). 